Sociedade Portuguesa de Psicologia da Saúde - SPPS - www.sp-ps.pt

DOI: http://dx.doi.org/10.15309/18psd190201

\title{
POSTTRAUMATIC GROWTH IN ADULT CANCER PATIENTS: AN UPDATED SYSTEMATIC REVIEW
}

\author{
Catarina Ramos (aramos@ispa.pt ${ }^{1,2 \square}$, Filipa Pimenta(filipa_pimenta@ispa.pt $)^{1}$, Ivone \\ Patrão(Ivone_patrao@ispa.pt) ${ }^{2,3}$, Margarida Costa (nana.bento.costa@gmail.com) ${ }^{3}$, Ana Isabel Santos \\ (anaicmlsantos@gmail.com) ${ }^{2}$, Tânia Rudnicki (tania.rudnicki@gmail.com) ${ }^{4}$, \& Isabel Leal (ileal@ispa.pt) ${ }^{1,2}$ \\ ${ }^{1}$ WJCR-William James Center for Research; ${ }^{2}$ ISPA - Instituto Universitário, Lisboa; ${ }^{3}$ Applied psychology research center \\ capabilities and Inclusion (APPsyCl); ${ }^{4}$ Capes Foundation Ministry of Education Of Brazil-Brasília/DF - Brazil; Centro \\ Universitário da Serra Gaúcha -FSG - Caxias do Sul/RS - Brazil.
}

\begin{abstract}
The current systematic review is an updated analysis of studies with adult cancer patients, regarding factors associated with posttraumatic growth (PTG), which is defined as perceived positive changes after traumatic event, such as cancer. A systematic review was conducted according to the PRISMA Statement guidelines. Seven electronic databases were searched. Quantitative studies with or without psychosocial group intervention that assessed PTG or similar construct (benefit finding [BF], positive life changes, stressrelated growth, growth) as main outcome were included. The initial systematic search yielded 659 papers, published between 2006 and 2015. From those, 81 studies fulfilled the inclusion criteria: 73 studies without intervention and 8 entailing an intervention program. The results suggested that socio-demographic (e.g. age, educational level, household income), clinical (e.g. stage of cancer), cognitive (e.g. intrusiveness, challenge to core beliefs), coping-related (e.g. positive reframing, religious coping) and other psychosocial variables (e.g. social support, optimism, spirituality) are positively associated with PTG. BF is associated with gender, marital status, cancer stage, both cancer and treatment type, positive active coping, positive reappraisal, social support and optimism. Psychosocial group interventions with cancer patients show significant effect on the increase of growth reported (PTG or BF). As conclusion, Growth following a cancer experience is an effect of several variables which might be targeted and promoted in the context of multidisciplinary teams, in hospital and clinical settings. Group interventions are a favorable context to the development of PTG after cancer, but interventions that assess PTG as primary outcome are still needed to evaluate the effect of group on PTG' facilitation.
\end{abstract}

Keywords: growth, posttraumatic growth, benefit finding, cancer

\section{CRESCIMENTO PÓS-TRAUMÁTICO EM ADULTOS COM CANCRO: UMA REVISÃO SISTEMÁTICA ATUALIZADA}

RESUMO: A presente revisão sistemática é uma análise atualizada de estudos com adultos com cancro, em relação aos fatores associados ao crescimento pós-traumático (CPT; posttraumatic growth), o qual é definido como mudanças positivas percebidas após o confronto com um acontecimento traumático como o cancro. Esta revisão sistemática foi

${ }^{\square}$ Rua Jardim do Tabaco, no 34. 1149-041 Lisboa, Portugal. e-mail: aramos@ispa.pt

www.sp-ps.pt 


\section{PTG IN ADULT CANCER PATIENTS: SYSTEMATIC REVIEW}

desenvolvida de acordo com PRISMA Statement guidelines. Sete bases de dados foram incluídas na pesquisa. Estudos quantitativos com ou sem intervenção em grupo que avaliaram o CPT ou constructo semelhante (benefit finding [benefícios percebidos, BP], positive life changes, stress-related growth, growth) como resultado principal, foram incluídos. De 659 artigos, publicados entre 2006 e 2015, 81 estudos preencheram os critérios de inclusão: 73 estudos sem intervenção e 8 estudos com programa de intervenção. Os resultados indicam que variáveis sócio-demográficas (e.g., idade, educação, estatuto sócio-económico), clínicas (e.g., estadio do cancro), cognitivas (e.g., pensamentos intrusivos, mudança de crenças centrais), relacionadas com o coping (e.g., reestruturação positiva, coping religioso) e outras variáveis psicossociais (e.g., apoio social, otimismo, espiritualidade) estão positivamente associadas ao CPT. Os BP estão associados ao género, ao estado civil, ao estadio do cancro, ao tipo de cancro, ao tipo de tratamento, ao coaching ativo positivo, à reavaliação positiva, ao apoio social e ao otimismo. As intervenções em grupo com pacientes com cancro apresentam um efeito significativo no aumento das mudanças positivas percebidas (CPT ou BP). Como conclusão, o crescimento psicológico após uma experiência de cancro pode resultar de várias variáveis que podem ser promovidas por equipas multidisciplinares em contextos hospitalares e clínicos. As intervenções em grupo são um contexto favorável ao desenvolvimento de CPT após o cancro, mas as intervenções que avaliam o CPT como resultado primário ainda são necessárias para avaliar o efeito do grupo para facilitar o CPT.

Palavras-chave: crescimento, crescimento pós-traumático, benefícios percebidos, cancro

Recebido em 22 de Fevereiro de 2018/ Aceite em 04 de Junho de 2018

During the last decades there has been an increase in the number of cancer diagnoses. According to World Health Organization (WHO, 2015), about 14 million new cases emerge every year, of which 8.2 million ultimately die. Having cancer represents an experience associated with multiple stressors. Due to a sudden and unexpected diagnosis, cancer can be a traumatic experience, which induces strong emotional responses, such as stress, anxiety, depression or Post-Traumatic Stress Disorder (PTSD) (Barskova \& Oesterreich, 2009).

Although the majority of studies have been focusing on the negative outcomes of cancer experience, there has been recent empirical studies showing a perception of positive changes after cancer. Posttraumatic Growth (PTG) has been recognized in the literature as the mainstream concept to define these positive changes, which accrue from the subject's attempts to cope with trauma (Tedeschi \& Calhoun, 1996; 2004).

Such as PTG, benefit finding (BF) is also another mainstream concept to define positive changes after trauma; however, they appear to be different constructs. According to Mols and colleagues (2009), BF develops immediately after the traumatic experience, whether PTG develops through time, since it is a product of successive rumination and cognitive restructuration. As a consequence, BF appears to be more superficial and fleeting, unlike PTG that changes the individual way of living and perceiving oneself (Harding et al., 2014). Other authors suggested the same idea, emphasizing that PTG originates selfrelated changes, unlike BF, which causes life style and behavioral changes (Koutroli et al., 2012; Lelorain et al., 2010). Being a complex and dynamic process, PTG occurs in interaction with multiple factors, which influence the subjective perception of the traumatic event (Tedeschi and Calhoun, 2004). The PTG model (Tedeschi \& Calhoun 1996; 2004) lists several variables as facilitators to the development of PTG, such as environmental characteristics (e.g. social support), event characteristics (e.g. duration), or coping strategies (e.g. problem-focused coping). Several empirical studies conducted with cancer patients are in line with this model, suggesting that PTG is predicted by the following variables: sociodemographic (e.g. age, educational level, income, marital status) (Bellizzi \& Blank, 2006; Cordova et al., 2007; Danhauer et 
al., 2013a; Llewellyn et al., 2013); clinical (e.g. stage, type of cancer, type of treatment) (Danhauer et al., 2013a; Thornton et al., 2012); psychological (e.g. anxiety, depression, PTSD) (Cordova et al., 2007; Thornton et al., 2012); physical (e.g. cortisol, immune function, physical exercise) (Diaz et al., 2014; Wang et al., 2014); cognitive (e.g. coping, rumination, core beliefs) (Bellizzi \& Blank, 2006; Danhauer et al., 2013b; Llewellyn et al., 2013; Thornton et al., 2012); social (e.g. social support, emotional disclosure) (Danhauer et al., 2013a; Llewellyn et al., 2013); and others such as optimism (Llewellyn et al., 2013), spirituality (Danhauer et al., 2013a), and religiosity (Thuné-Boyle et al., 2011).

Even though there has been empirical evidence about which factors are PTG' predictors in cancer patients, inconsistencies remain relatively to the predictive value of some factors towards PTG, such as PTSD symptoms for example (Cordova et al., 2007; Morrill et al., 2008). In an effort to shed light on this construct, some systematic reviews have been conducted (Casellas-Grau, Font, \& Vives, 2014; Casellas-Grau, Ochoa, \& Ruini, 2017; Harding et al., 2014; Kolokotroni et al., 2014; Koutroli et al., 2012; Shand et al., 2015; Stanton et al., 2006). However, these inconsistencies are still to be clarified in the light of current empirical and intervention studies.

Hence, there is still a need to further systematize the available results in order to offer both clinicians and researchers a better understanding about the predictors of the development of personal growth in the aftermath of cancer, and new evidences of relationships between personal growth after cancer and psychological and physical variables that, until now, were not covered by past studies. Also, we intend to explore some aspects neglected by previous systematic reviews, such as: a) the inclusion of studies with similar concepts, for example BF, stress-related growth, and positive life changes, since the independence of these concepts and PTG has not been fully demonstrated (Kolokotroni et al., 2014; Koutroli et al., 2012); b) the inclusion of different types of cancer, since the previous reviews only included a specific type of cancer such as breast (Kolokotroni et al., 2014; Koutroli et al., 2012) or head and neck cancer (Harding et al., 2014); c) the inclusion of intervention studies in addition to empirical studies (Casellas-Grau et al., 2014; 2017; Harding et al., 2014; Kolokotroni et al., 2014; Shand et al., 2015); and d) the assessment of risk of bias of the empirical articles (Kolokotroni et al., 2014; Koutroli et al., 2012).

With the purpose to fill these gaps, this updated review will include studies with both PTG and BF (or similar constructs defining the perceived positive changes after a traumatic event) and will assess the quality of the included studies. The objectives of the current systematic review are as follows: to analyze the presence of growth in patients with the diagnosis of cancer; to explore the relationship between growth and clinical, sociodemographic, and psychosocial variables; to discuss the perception about positive changes during the course of different types of cancer; and to contribute to enlarge the scientific and clinical knowledge about PTG in cancer patients.

\section{METHOD}

This systematic review was developed according to APA's Meta-Analysis Reporting Method (APA Publications and Communications Board Working Group on Journal Article Reporting Standards, 2008) and Preferred Reporting Itens for Systematic Reviews and Meta-Analyses (PRISMA) Statement guidelines (Liberati et al., 2009); and had the review record CRD420103012 on the PROSPERO register.

\section{Inclusion and Exclusion Criteria}

Eligible studies were original, published, and empirical (with or without intervention) studies, that had assessed growth in cancer patients and had examined the relationship between growth and at least one socio-demographic, psychological or social variable. Cross-sectional or longitudinal studies were eligible for inclusion as well as quantitative studies, randomized controlled trials (studies with an intervention) and comparative studies. English, French, Spanish and Portuguese papers were included. Additional 


\section{PTG IN ADULT CANCER PATIENTS: SYSTEMATIC REVIEW}

inclusion criteria were the following: In primary studies, positive changes were assessed through the construct of PTG, BF, positive life changes, adversarial growth, or stress-related growth; Growth was evaluated with a valid measure (e.g. Posttraumatic Growth Inventory - original or short form; Benefit Finding Scale); PTG (or similar construct) was defined as primary outcome in empirical studies and as primary or secondary outcome in intervention studies; Study participants were adult patients who had been diagnosed with any type of cancer (e.g. breast, prostate, colon, etc.), who were in phase of diagnosis, treatment, or surveillance. Randomized controlled trials that included any type of psychosocial intervention, conducted by a health professional (e.g. psychologist, nurse, physiotherapist); Individual or group interventions, targeting patients (who adhere individually or with a partner/spouse or other family member) were also included.

Conversely, the exclusion criteria were the following: qualitative studies; mixed methods; metaanalysis, systematic and literature reviews; unpublished researches; book chapters, commentaries and editorials, thesis, or abstracts from Congresses' presentations; studies free of intervention, that assessed PTG as a secondary outcome or as a mediator variable were excluded; However, since most interventions did not directly focus PTG's development, this systematic review included intervention studies which had PTG as a secondary outcome. Studies that measured PTG through open questions and not through valid measures (e.g. PTGI) and studies with interventions only with family members of a cancer patient were also not considered. Articles in which samples included cancer patients in addition to patients from other diseases were also not included in this systematic review. Exclusion criteria related to individual characteristics (e.g. gender or ethnicity) and cancer-related characteristics (e.g. stage, surgery, or metastasis) were not used.

It is important to note that, overlapping samples were found: when different papers reported separate results regarding the same sample or substantially overlapping samples, the distinct papers were assumed as one single study, counting as one entry (e.g. Ruini et al., 2013; 2014).

\section{Search Strategy}

Studies were identified by searching multiple literature databases related to health, medicine and psychology, such as MEDLINE, PsychArticles, PsycINFO, PubMed, Scielo, PePsic, and Web of Science. We restricted the search to studies published between January 2006 and May 2015, since the last systematic review with adult cancer patients had included studies up to the year 2005 (Stanton et al., 2006). The selection of studies for eligibility and data extraction were performed by five independent researchers and possible disagreements were discussed and solved between them.

To identify papers addressing growth and cancer the following search terms were used: posttraumatic growth; growth; benefit finding; positive life changes; stress-related growth; cancer; oncological disease; neoplasm; tumor; and carcinoma. In order to select the articles that met the inclusion criteria and to exclude the others that did not meet them, the titles and abstracts were examined. If necessary, and in order to clarify any information, the full papers were also examined.

In order to avoid source selection bias and to ensure an exhaustive and comprehensive search procedure, additional search strategies were applied such as searching of scientific journals which had published relevant articles in this area, analyzing the reference list of primary studies, and exploring other databases such as national library databases.

\section{Search Results}

The initial searches from the databases identified 659 potentially relevant studies. After the examination of the titles, abstracts and full articles, we excluded papers based on inclusion criteria mentioned above. Thus, a total of 578 studies were excluded because they were systematic reviews and/or meta-analysis, literature reviews, theoretical articles or commentaries (56); were chapters, books, or abstracts from presentations in conferences (141); were thesis or dissertations (55); used qualitative or 
mixed methodology (70); were study protocols (4); assessed psychometric properties or validated a measure that assessed PTG or similar construct (22); were non-randomized trials or non-experimental studies (6); used only caregivers or family members of patients, as sample (40); used samples consisted of children or adolescents that suffered from cancer (20); were papers written in languages other than the ones mentioned in the inclusion criteria (24); assessed only medical outcomes or PTG as a result of a medical procedure (27); did not measure PTG (or similar construct), PTG was not assessed as primary outcome or was assessed as a mediator variable (105); used open questions to measure PTG, did not use one of the main growth measures or used changed versions (without previous validation) of the measure (8). Figure 1 displays a flowchart of studies.

\section{Studies identified through database searching: 659}

Full-text studies assessed for eligibility: 154

Fig. 1. Flowchart of studies.

\section{Studies excluded: $\mathbf{5 0 5}$}

systematic reviews and/or meta-analysis, literature review, theoretical article or commentary (55);

chapters, books or abstracts from presentations in conferences

(141); dissertations (55);

qualitative or mixed methods (65);

study protocols (4);

validation of an instrument (20);

non-randomized trials (4);

family members of patients (37);

adolescents or young adults (17);

other languages (24);

medical outcomes (24);

PTG was not measured, PTG was not assessed as primary

outcome or was assessed as mediator variable (59).

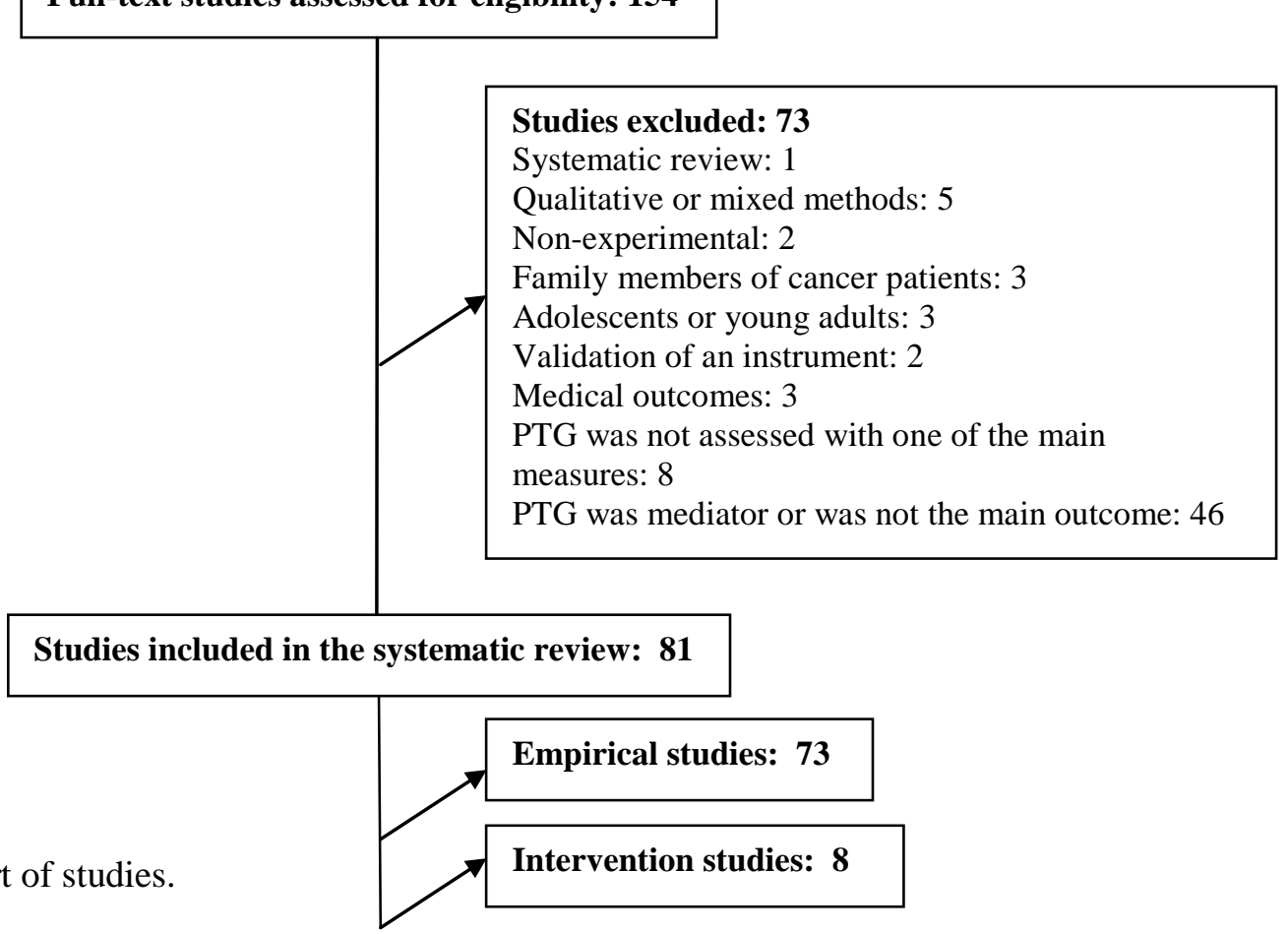




\section{PTG IN ADULT CANCER PATIENTS: SYSTEMATIC REVIEW}

\section{Quality Assessment}

The final sample consists of 81 eligible studies and the general quality of each study was assessed using a 29-item check-list adapted from the Quality Assessment Tool - Cochrane's Handbook (Higgins \& Green, 2011). Accordingly, the quality from each study was assessed by the evaluation of the following items: 1) introduction (e.g., background of the existing literature); 2) objectives (description of objectives and/or hypothesis of the study); 3) study design; 4) sampling process; 5) participants' recruitment; 6) sample size calculation; 7) inclusion and exclusion criteria; 8) data collection locals (name and/or other characteristics of data collection locals); 9) ethic committee' approval; 10) differences between groups (description of the identification and/or resolution of the differences between groups); 11) identification of the existent conditions/groups - e.g. control vs. treatment; 12) randomization method description; 13) description of the intervention for the experimental group; 14) description of the intervention for the control/alternative group; 15) study' costs; 16) assessments (description of how, who and where were carried out); 17) blind assessment; 18) drop-outs (numbers and/or reasons for drop-outs); 19) sociodemographic characteristics; 20) cancer-related characteristics; 21) measures (description and/or psychometric properties); 22) statistical analysis; 23) results (detailed and adequate description of the results); 24) discussion (literature-based discussion of the results); 25) generalization (or not) of the results; 26) limitations; 27) registration of the intervention program; 28) sources of funding; and 29) conflict of interests. It is important to note that the items 12, 13, 14, and 27 were exclusive to studies that encompassed a group intervention.

The majority of the 29 items was scored through a scale consisted of three points from 0 to 2: 0 (not done / or not reported), 1 (done but unclear and /or reported to some extend), 2 (adequately done and/ or adequately reported) (Higgins \& Green, 2011). However, five items were scored from 0 to 3, since they accumulated more than one aspect needed to be assessed in the context of quality evaluation; an example of this was the item that assessed the quality of the measures' report ( 0 - not done; 1 - done but not clear; 2- reported without psychometric characteristics; 3-reported, including psychometric characteristics).

Five researchers independently assessed the quality of the included studies. The inter-rater agreement between pairs of two researchers was calculated on 65 papers $(80 \%)$ through the Cohen's Kappa and the averages were good, as following: $.966 ; .963 ; .943 ; .898 ; .801$. Disagreements in quality assessment were resolved by consensus between pairs of two researchers. Remain divergences were clarified by the researcher CR.

A summary of the quality assessment is presented in table 1 for cross-sectional studies, and in table 2, for longitudinal and intervention studies. 
Table 1.

Quality assessment of cross-sectional studies

\begin{tabular}{|c|c|c|c|c|c|c|c|c|c|c|c|c|c|c|c|c|c|c|c|c|c|c|c|}
\hline Article & 1 & 2 & 3 & 4 & 5 & 6 & 7 & 8 & 9 & 15 & 16 & 18 & 19 & 20 & 21 & 22 & 23 & 24 & 25 & 26 & 28 & 29 & Total \\
\hline Andrykowski et al. [2] & 2 & 3 & 1 & 2 & 2 & 0 & 2 & 2 & 2 & 2 & 1 & 0 & 2 & 1 & 2 & 2 & 2 & 2 & 0 & 2 & 2 & 0 & 34 \\
\hline Baník \& Gajdošová [4] & 2 & 2 & 0 & 1 & 2 & 0 & 2 & 1 & 2 & 0 & 2 & 0 & 2 & 2 & 2 & 2 & 2 & 2 & 0 & 2 & 0 & 0 & 28 \\
\hline Bellizzi \& Blank [5] & 2 & 3 & 2 & 2 & 2 & 2 & 0 & 2 & 2 & 2 & 2 & 3 & 2 & 2 & 3 & 1 & 2 & 2 & 2 & 2 & 2 & 0 & 42 \\
\hline Bellizzi et al. [6] & 2 & 3 & 2 & 2 & 1 & 0 & 0 & 2 & 2 & 0 & 1 & 3 & 2 & 2 & 3 & 2 & 2 & 2 & 2 & 2 & 0 & 0 & 35 \\
\hline Bozo et al. [7] & 2 & 3 & 2 & 2 & 1 & 0 & 0 & 2 & 2 & 0 & 2 & 0 & 2 & 2 & 3 & 1 & 2 & 2 & 1 & 2 & 0 & 0 & 31 \\
\hline Büyükaçik-Çolak et al. [10] & 2 & 2 & 2 & 2 & 0 & 0 & 0 & 1 & 1 & 0 & 0 & 0 & 1 & 2 & 3 & 0 & 1 & 2 & 0 & 0 & 0 & 0 & 19 \\
\hline Cavell et al. [12] & 1 & 2 & 2 & 2 & 1 & 0 & 2 & 2 & 2 & 0 & 1 & 2 & 2 & 2 & 1 & 1 & 2 & 2 & 2 & 2 & 0 & 0 & 31 \\
\hline Chan et al. [13] & 2 & 3 & 2 & 2 & 1 & 0 & 2 & 2 & 2 & 0 & 0 & 0 & 2 & 2 & 3 & 2 & 2 & 2 & 2 & 1 & 0 & 0 & 32 \\
\hline Cohen, \& Numa [14] & 2 & 3 & 2 & 2 & 2 & 0 & 0 & 2 & 2 & 2 & 2 & 0 & 2 & 2 & 3 & 2 & 2 & 2 & 2 & 2 & 0 & 0 & 36 \\
\hline Cordova et al. [15] & 2 & 3 & 2 & 2 & 2 & 0 & 2 & 2 & 2 & 2 & 2 & 3 & 2 & 2 & 3 & 2 & 2 & 2 & 2 & 2 & 2 & 0 & 43 \\
\hline Cormio et al. [16] & 2 & 2 & 2 & 2 & 1 & 0 & 2 & 2 & 2 & 0 & 2 & 3 & 2 & 1 & 2 & 1 & 2 & 2 & 1 & 2 & 0 & 0 & 33 \\
\hline Crawford et al. [17] & 2 & 3 & 2 & 2 & 1 & 0 & 2 & 2 & 2 & 0 & 2 & 3 & 2 & 2 & 3 & 2 & 2 & 2 & 0 & 2 & 0 & 2 & 38 \\
\hline Diaz et al. [21] & 2 & 3 & 2 & 2 & 2 & 0 & 2 & 2 & 0 & 0 & 1 & 2 & 2 & 1 & 3 & 2 & 2 & 1 & 2 & 2 & 2 & 2 & 37 \\
\hline Dunn et al. [22] & 2 & 3 & 2 & 2 & 2 & 0 & 2 & 2 & 2 & 0 & 2 & 1 & 1 & 1 & 3 & 2 & 2 & 2 & 0 & 2 & 0 & 2 & 35 \\
\hline Garland et al. [23] & 2 & 3 & 2 & 2 & 1 & 0 & 2 & 2 & 2 & 0 & 2 & 3 & 2 & 2 & 2 & 2 & 2 & 2 & 0 & 1 & 2 & 2 & 38 \\
\hline Harrington et al. [25] & 2 & 3 & 2 & 2 & 1 & 0 & 3 & 1 & 1 & 0 & 1 & 3 & 2 & 2 & 3 & 1 & 2 & 2 & 2 & 2 & 0 & 0 & 35 \\
\hline Heidarzadeh et al. [27] & 2 & 2 & 2 & 2 & 1 & 0 & 2 & 2 & 0 & 0 & 1 & 0 & 2 & 2 & 3 & 1 & 2 & 2 & 0 & 2 & 0 & 2 & 30 \\
\hline Ho et al. [28] & 2 & 3 & 2 & 2 & 1 & 0 & 0 & 2 & 2 & 0 & 2 & 0 & 2 & 2 & 3 & 1 & 2 & 2 & 2 & 2 & 2 & 0 & 34 \\
\hline Ho et al. [29] & 2 & 3 & 2 & 2 & 2 & 0 & 2 & 2 & 2 & 0 & 1 & 2 & 2 & 2 & 3 & 2 & 2 & 2 & 1 & 2 & 0 & 2 & 38 \\
\hline Kangas et al. [31] & 2 & 3 & 2 & 1 & 1 & 0 & 1 & 2 & 2 & 0 & 1 & 0 & 3 & 2 & 2 & 2 & 1 & 2 & 2 & 0 & 2 & 0 & 31 \\
\hline Karanci \& Erkam [32] & 2 & 2 & 2 & 2 & 2 & 0 & 2 & 2 & 0 & 2 & 2 & 3 & 2 & 2 & 3 & 1 & 2 & 2 & 0 & 0 & 0 & 0 & 33 \\
\hline Kinsinger et al. [34] & 2 & 2 & 2 & 2 & 2 & 0 & 2 & 2 & 1 & 0 & 2 & 0 & 2 & 2 & 3 & 1 & 2 & 2 & 2 & 2 & 2 & 0 & 35 \\
\hline Lelorain et al. [37] & 2 & 3 & 2 & 2 & 2 & 0 & 3 & 2 & 2 & 0 & 2 & 3 & 2 & 2 & 3 & 2 & 2 & 2 & 1 & 2 & 2 & 0 & 41 \\
\hline Li et al. [38] & 2 & 1 & 2 & 2 & 2 & 0 & 0 & 2 & 2 & 0 & 1 & 0 & 1 & 1 & 3 & 0 & 2 & 2 & 0 & 2 & 0 & 0 & 25 \\
\hline Martins da Silva et al. [41] & 2 & 3 & 2 & 2 & 2 & 0 & 2 & 2 & 2 & 0 & 2 & 3 & 2 & 2 & 3 & 2 & 2 & 2 & 1 & 2 & 1 & 0 & 39 \\
\hline Mols et al. [43] & 2 & 2 & 2 & 2 & 2 & 0 & 2 & 2 & 0 & 0 & 2 & 1 & 0 & 2 & 0 & 2 & 2 & 2 & 2 & 2 & 2 & 0 & 31 \\
\hline Morris \& Shakespeare-Finch [44] & 2 & 3 & 2 & 1 & 1 & 0 & 0 & 1 & 2 & 0 & 1 & 0 & 2 & 2 & 3 & 2 & 2 & 2 & 2 & 2 & 0 & 0 & 30 \\
\hline Morris \& Shakespeare-Finch [45] & 2 & 3 & 2 & 2 & 2 & 0 & 0 & 1 & 1 & 0 & 2 & 2 & 2 & 1 & 3 & 2 & 2 & 2 & 0 & 2 & 0 & 0 & 31 \\
\hline Morris et al. [46] & 2 & 3 & 2 & 2 & 1 & 0 & 0 & 2 & 0 & 0 & 1 & 2 & 2 & 1 & 3 & 1 & 2 & 2 & 0 & 1 & 0 & 0 & 27 \\
\hline Mystakidou et al. [47] & 1 & 2 & 2 & 2 & 0 & 0 & 2 & 2 & 2 & 0 & 0 & 2 & 2 & 1 & 3 & 1 & 2 & 2 & 0 & 2 & 0 & 0 & 28 \\
\hline Mystakidou et al. [48] & 2 & 3 & 2 & 2 & 1 & 0 & 2 & 1 & 2 & 2 & 2 & 3 & 2 & 2 & 3 & 2 & 2 & 2 & 0 & 2 & 0 & 2 & 39 \\
\hline Rahmani et al. [52] & 2 & 2 & 2 & 1 & 1 & 0 & 2 & 1 & 2 & 0 & 2 & 0 & 2 & 1 & 3 & 1 & 2 & 2 & 2 & 2 & 2 & 0 & 32 \\
\hline Rand et al. [53] & 1 & 2 & 2 & 2 & 1 & 0 & 2 & 2 & 0 & 2 & 2 & 2 & 1 & 1 & 3 & 2 & 2 & 2 & 0 & 2 & 2 & 0 & 33 \\
\hline Ruini et al. [54] & 2 & 3 & 2 & 2 & 2 & 0 & 2 & 1 & 1 & 2 & 2 & 3 & 2 & 1 & 3 & 2 & 2 & 2 & 0 & 2 & 0 & 0 & 36 \\
\hline Ruini et al. [55] & 2 & 3 & 2 & 2 & 1 & 0 & 1 & 2 & 2 & 2 & 2 & 3 & 2 & 1 & 3 & 2 & 2 & 2 & 1 & 2 & 0 & 2 & 39 \\
\hline Schmidt et al. [57] & 1 & 2 & 2 & 2 & 2 & 0 & 2 & 1 & 1 & 0 & 2 & 2 & 2 & 1 & 3 & 2 & 2 & 2 & 2 & 2 & 0 & 0 & 33 \\
\hline Schroevers et al. [58] & 1 & 2 & 2 & 2 & 1 & 0 & 0 & 2 & 0 & 0 & 2 & 2 & 2 & 2 & 3 & 2 & 2 & 2 & 2 & 2 & 0 & 0 & 31 \\
\hline Schroevers \& Teo [59] & 2 & 3 & 2 & 2 & 2 & 0 & 0 & 1 & 1 & 0 & 2 & 0 & 2 & 1 & 3 & 2 & 2 & 2 & 2 & 2 & 0 & 0 & 31 \\
\hline Smith et al. [63] & 2 & 3 & 2 & 2 & 2 & 0 & 2 & 2 & 0 & 0 & 1 & 3 & 2 & 2 & 3 & 0 & 2 & 2 & 2 & 2 & 0 & 0 & 34 \\
\hline Smith et al. [64] & 2 & 3 & 2 & 2 & 2 & 0 & 2 & 2 & 2 & 2 & 1 & 0 & 2 & 2 & 3 & 2 & 2 & 2 & 1 & 2 & 2 & 0 & 38 \\
\hline Soo \& Sherman [65] & 2 & 3 & 2 & 2 & 2 & 0 & 1 & 2 & 2 & 0 & 2 & 0 & 2 & 2 & 3 & 2 & 2 & 2 & 2 & 2 & 2 & 2 & 39 \\
\hline Strack et al. [67] & 1 & 3 & 2 & 2 & 0 & 0 & 0 & 1 & 0 & 2 & 1 & 0 & 1 & 0 & 2 & 1 & 2 & 1 & 0 & 0 & 0 & 0 & 19 \\
\hline Svetina \& Nastran [68] & 1 & 2 & 2 & 2 & 2 & 0 & 0 & 2 & 2 & 0 & 1 & 3 & 2 & 2 & 3 & 2 & 2 & 2 & 0 & 0 & 0 & 2 & 32 \\
\hline Tanriverd et al. [70] & 1 & 2 & 2 & 2 & 0 & 0 & 0 & 2 & 2 & 0 & 2 & 3 & 2 & 0 & 3 & 0 & 1 & 1 & 0 & 0 & 0 & 0 & 23 \\
\hline Tanyi et al. [72] & 2 & 2 & 2 & 2 & 1 & 0 & 2 & 2 & 2 & 0 & 2 & 0 & 2 & 2 & 3 & 2 & 2 & 2 & 1 & 2 & 2 & 2 & 37 \\
\hline
\end{tabular}


PTG IN ADULT CANCER PATIENTS: SYSTEMATIC REVIEW

Thombre et al. [73]

Thuné-Boyle et al. [75]

Wang et al. [78]

Wilson et al. [80]

Yu et al. [81]

0 - not done / or not reported; 1- done but unclear and /or reported to some extend; 2- adequately done and/ or adequately reported.

Table 2.

Quality assessment of longitudinal and intervention studies

\begin{tabular}{|c|c|c|c|c|c|c|c|c|c|c|c|c|c|c|c|c|c|c|c|c|c|c|c|c|c|c|c|c|c|c|}
\hline Article & 1 & 2 & 3 & 4 & 5 & 6 & 7 & 8 & 9 & 10 & 11 & 12 & 13 & 14 & 15 & 16 & 17 & 18 & 19 & 20 & 21 & 22 & 23 & 24 & 25 & 26 & 27 & 28 & 29 & $\begin{array}{c}\text { Weighted } \\
\text { mean }\end{array}$ \\
\hline Abdullah et al. [1] & 2 & 2 & 2 & 2 & 1 & 0 & 3 & 1 & 2 & 2 & & & & & 0 & 1 & & 2 & 2 & 2 & 3 & 2 & 2 & 2 & 0 & 2 & & 2 & 2 & 49 \\
\hline Antoni et al. [3] & 1 & 3 & 2 & 2 & 1 & 0 & 2 & 1 & 0 & 0 & $\overline{2}$ & $\overline{0}$ & $\overline{2}$ & $\overline{2}$ & 0 & 2 & $\overline{0}$ & 3 & 2 & 2 & 2 & 2 & 2 & 2 & 2 & 2 & $\overline{0}$ & 2 & 0 & 41 \\
\hline Brix et al. [8] & 2 & 3 & 2 & 2 & 1 & 0 & 2 & 1 & 0 & - & - & $\ldots$ & $\ldots$ & _- & 0 & 2 & - & 3 & 2 & 2 & 2 & 2 & 2 & 2 & 2 & 2 & - & 2 & 2 & 50 \\
\hline Bussell \& Naus [9] & 2 & 3 & 2 & 2 & 1 & 0 & 0 & 2 & 1 & - & - & - & - & - & 0 & 1 & - & 0 & 2 & 2 & 3 & 2 & 2 & 2 & 0 & 2 & - & 0 & 0 & 38 \\
\hline Cameron et al. [11] & 2 & 3 & 2 & 0 & 1 & 0 & 2 & 2 & 2 & - & $\overline{2}$ & - & - & - & 0 & 2 & - & 3 & 2 & 2 & 2 & 2 & 2 & 2 & 2 & 2 & - & 2 & 0 & 49 \\
\hline Danhauer et al. [18] & 2 & 3 & 2 & 2 & 1 & 0 & 2 & 2 & 0 & $\overline{0}$ & - & - & - & - & 0 & 1 & - & 2 & 2 & 2 & 3 & 2 & 2 & 2 & 2 & 2 & - & 2 & 0 & 45 \\
\hline Danhauer et al. [19] & 2 & 3 & 2 & 2 & 2 & 0 & 2 & 2 & 2 & _ & - & - & - & $\overline{-}$ & 1 & 2 & - & 1 & 2 & 2 & 3 & 2 & 2 & 2 & 2 & 2 & - & 2 & 2 & 55 \\
\hline Danhauer et al. [20] & 2 & 3 & 2 & 2 & 2 & 0 & 2 & 2 & 0 & - & - & - & - & - & 0 & 1 & - & 3 & 2 & 1 & 1 & 2 & 2 & 2 & 0 & 2 & - & 2 & 2 & 46 \\
\hline Garlick et al. [24] & 2 & 3 & 2 & 2 & 2 & 0 & 3 & 1 & 0 & $\overline{0}$ & $\overline{0}$ & - & $\overline{2}$ & $\overline{0}$ & 0 & 2 & $\overline{0}$ & 0 & 2 & 1 & 2 & 2 & 2 & 2 & 1 & 2 & $\overline{0}$ & 2 & 0 & 35 \\
\hline Hawkes et al. [26] & 2 & 3 & 2 & 2 & 1 & 0 & 2 & 2 & 2 & 0 & 0 & $\overline{1}$ & 2 & 0 & 0 & 1 & 0 & 0 & 2 & 1 & 2 & 2 & 2 & 2 & 1 & 2 & 2 & 2 & 2 & 40 \\
\hline Kállay \& Baban [30] & 2 & 2 & 2 & 0 & 1 & 0 & 0 & 2 & 0 & _ & - & - & - & _- & 0 & 1 & - & 1 & 1 & 1 & 2 & 1 & 1 & 1 & 0 & 2 & - & 0 & 0 & 26 \\
\hline Kent et al. [33] & 2 & 3 & 2 & 2 & 1 & 0 & 2 & 2 & 2 & $\overline{0}$ & - & - & - & - & 0 & 1 & - & 3 & 2 & 2 & 3 & 2 & 2 & 2 & 2 & 2 & - & 2 & 2 & 52 \\
\hline Labelle et al. [35] & 2 & 2 & 2 & 2 & 2 & 0 & 2 & 1 & 2 & 2 & $\overline{1}$ & - & - & - & 0 & 2 & $\overline{0}$ & 3 & 2 & 2 & 2 & 3 & 2 & 2 & 2 & 2 & $\overline{0}$ & 2 & 0 & 47 \\
\hline Lechner et al. [36] & 2 & 2 & 2 & 2 & 1 & 0 & 0 & 1 & 0 & - & $\ldots$ & - & - & - & 0 & 1 & - & 2 & 2 & 2 & 1 & 1 & 2 & 2 & 2 & 2 & $\ldots$ & 2 & 0 & 38 \\
\hline Liu et al. [39] & 2 & 3 & 2 & 2 & 2 & 1 & 2 & 2 & 2 & - & - & $\overline{1}$ & - & - & 0 & 1 & - & 2 & 2 & 2 & 3 & 2 & 2 & 2 & 1 & 2 & - & 1 & 2 & 52 \\
\hline Llewellyn et al. [40] & 1 & 2 & 2 & 2 & 0 & 0 & 3 & 1 & 1 & - & - & - & - & - & 0 & 1 & - & 3 & 0 & 0 & 3 & 2 & 2 & 2 & 0 & 2 & - & 2 & 0 & 38 \\
\hline McDonough et al. [42] & 2 & 3 & 2 & 1 & 2 & 0 & 2 & 0 & 1 & - & - & - & - & - & 0 & 1 & - & 0 & 1 & 2 & 3 & 2 & 2 & 2 & 2 & 2 & - & 2 & 0 & 42 \\
\hline Park et al. [49] & 2 & 3 & 2 & 2 & 2 & 0 & 2 & 2 & 0 & - & - & - & - & - & 0 & 2 & - & 2 & 2 & 1 & 3 & 2 & 2 & 2 & 2 & 2 & - & 2 & 0 & 49 \\
\hline Pat- Horenczyk et al. [50] & 1 & 3 & 2 & 0 & 2 & 0 & 2 & 2 & 1 & - & - & - & $\overline{2}$ & $\overline{2}$ & 0 & 1 & - & 2 & 2 & 2 & 3 & 2 & 2 & 1 & 1 & 2 & - & 0 & 0 & 42 \\
\hline Posluszny et al. [51] & 2 & 1 & 2 & 2 & 1 & 0 & 2 & 2 & 2 & $\overline{1}$ & $\overline{1}$ & - & $\ldots$ & - & 1 & 1 & - & 1 & 2 & 2 & 3 & 2 & 2 & 2 & 1 & 2 & - & 0 & 0 & 42 \\
\hline Salsman, et al. [56] & 2 & 3 & 2 & 2 & 2 & 0 & 3 & 2 & 2 & _ & - & - & - & - & 2 & 2 & - & 3 & 2 & 2 & 3 & 2 & 2 & 2 & 2 & 2 & - & 0 & 0 & 55 \\
\hline Schultz \& Mohamed [60] & 2 & 1 & 2 & 2 & 1 & 0 & 1 & 1 & 0 & - & - & - & - & - & 0 & 1 & - & 2 & 1 & 1 & 3 & 1 & 2 & 2 & 0 & 0 & - & 0 & 0 & 30 \\
\hline Scrignaro et al. [61] & 2 & 3 & 2 & 2 & 1 & 0 & 2 & 1 & 2 & - & - & - & - & - & 0 & 1 & - & 3 & 2 & 2 & 3 & 2 & 2 & 2 & 2 & 2 & - & 0 & 0 & 48 \\
\hline Silva et al. [62] & 2 & 3 & 2 & 2 & 1 & 1 & 2 & 1 & 2 & $\overline{2}$ & - & - & - & - & 0 & 1 & - & 3 & 1 & 1 & 3 & 1 & 2 & 2 & 1 & 2 & - & 0 & 0 & 44 \\
\hline Stafford et al. [66] & 2 & 3 & 2 & 2 & 2 & 0 & 2 & 1 & 1 & _ & - & - & $\overline{2}$ & - & 0 & 1 & $\overline{0}$ & 2 & 2 & 2 & 3 & 2 & 2 & 2 & 0 & 2 & $\overline{0}$ & 2 & 1 & 44 \\
\hline Tang et al. [69] & 2 & 2 & 2 & 2 & 2 & 0 & 2 & 1 & 2 & $\overline{3}$ & - & - & - & - & 0 & 1 & - & 3 & 2 & 1 & 2 & 2 & 2 & 2 & 2 & 2 & - & 2 & 2 & 52 \\
\hline Tanyi et al. [71] & 2 & 3 & 2 & 2 & 2 & 0 & 2 & 2 & 2 & 2 & - & - & - & - & 1 & 2 & - & 2 & 2 & 2 & 3 & 2 & 2 & 2 & 2 & 2 & - & 0 & 0 & 52 \\
\hline Thornton et al. [74] & 2 & 3 & 1 & 2 & 2 & 0 & 3 & 2 & 2 & - & - & - & - & - & 0 & 2 & - & 3 & 2 & 2 & 3 & 2 & 2 & 2 & 0 & 2 & - & 2 & 2 & 54 \\
\hline Tomich \& Helgeson [76] & 2 & 3 & 2 & 2 & 1 & 0 & 1 & 1 & 2 & - & - & $\overline{1}$ & - & - & 0 & 1 & $\overline{1}$ & 3 & 2 & 2 & 3 & 2 & 2 & 2 & 1 & 2 & $\overline{1}$ & 2 & 0 & 45 \\
\hline Wang et al. [77] & 2 & 2 & 2 & 2 & 1 & 0 & 2 & 1 & 1 & 1 & - & - & - & - & 2 & 2 & - & 2 & 2 & 2 & 3 & 2 & 2 & 2 & 2 & 2 & - & 2 & 0 & 49 \\
\hline Wang et al. [79] & 2 & 2 & 2 & 2 & 1 & 0 & 3 & 1 & 2 & & & - & - & & 0 & 1 & - & 2 & 2 & 2 & 3 & 2 & 2 & 2 & 0 & 2 & - & 2 & 2 & 49 \\
\hline
\end{tabular}

Wang

0 - not done / or not reported; 1 - done but unclear and /or reported to some extend; 2 - adequately done and/ or adequately reported. 


\section{Data Extraction}

Both study selection procedure and data extraction were carried out by the five independent researchers. Discrepancies related to the data extraction were discussed between the five researchers in consensus meetings.

Table 3 in the Supplementary Material summarizes the main characteristics of the 73 non-intervention studies: a) study (authors, date); b) number of participants; c) cancer type; d) cancer stage; e) time since diagnosis; f) design; g) measure of growth (i.e., instrument used to assess growth and mean and standard deviation of growth); h) other variables (namely, additional variables assessed and respective measures used to evaluate each of them); i) main outcomes (factors associated with or predictors of growth). These characteristics were selected in order to advance the understanding of the relations between growth and sociodemographic, psychological and social variables among adult patients diagnosed with cancer. The intervention studies were characterized regarding the same features; additionally the type of the intervention was included, as shown in table 4 in the Supplementary Material.

Table 3.

Characteristics of empirical studies without intervention

\begin{tabular}{|c|c|c|c|c|c|c|c|c|c|}
\hline $\begin{array}{c}\text { Study } \\
\text { (Authors, } \\
\text { date) }\end{array}$ & $n$ & Cancer & $\begin{array}{l}\text { Time } \\
\text { since } \\
\text { cancer }\end{array}$ & Design & Scale & $\begin{array}{l}\text { Total score } \\
\text { M (SD) }\end{array}$ & Variable & Scale & $\begin{array}{l}\text { Outcomes (Factors associated with } \\
\text { growth) }\end{array}$ \\
\hline $\begin{array}{l}\text { Abdullah et } \\
\text { al. [1] }\end{array}$ & $\begin{array}{l}50(33 \\
\text { men; } 17 \\
\text { women })\end{array}$ & $\begin{array}{l}\text { Head and } \\
\text { neck } \\
\text { (Stage I- } \\
\text { IV) }\end{array}$ & $\begin{array}{l}\text { Range 0- } \\
12 \\
\text { months }\end{array}$ & $\begin{array}{l}\text { Longitudinal } \\
\text { T1 = within } 1 \\
\text { year diagnosis; } \\
\text { T2 = } 6 \text { months } \\
\text { after } \mathrm{T} 1\end{array}$ & $\begin{array}{l}\text { PTGI } \\
- \text { SF }\end{array}$ & $\mathrm{Nr}$ & $\begin{array}{l}\text { Gender, age, monthly } \\
\text { income, education } \\
\text { status, diagnosis, } \\
\text { stage of cancer, } \\
\text { treatment, anxiety } \\
\text { and depression }\end{array}$ & $\mathrm{HADS}^{1}$ & $\begin{array}{l}\text { The correlations between PTG, } \\
\text { depression and anxiety were not } \\
\text { significant at T1. } \\
\text { Anxiety and depression did not predict } \\
\text { PTG, longitudinally. }\end{array}$ \\
\hline $\begin{array}{l}\text { Andrykows } \\
\text { ki et al. [2] }\end{array}$ & $\begin{array}{l}242 \\
(190 \\
\text { lung } \\
\text { cancer } \\
\text { survivor } \\
\text { s (LC); } \\
152 \\
\text { healthy } \\
\text { controls } \\
(\text { HC) }) \\
\end{array}$ & $\begin{array}{l}\text { Lung } \\
\text { (Stage nr) }\end{array}$ & $\begin{array}{l}M=15,7 \\
\text { months; } \\
S D=2.30 \\
\text { months }\end{array}$ & $\begin{array}{l}\text { Cross- } \\
\text { sectional }\end{array}$ & PTGI & $\begin{array}{l}\mathrm{LC}(M= \\
50.1 ; S D= \\
28.3) ; \mathrm{HC}(M \\
=38.5 ; S D= \\
30.3)\end{array}$ & Physical comorbidity & List of items & $\begin{array}{l}\text { The LC group reported greater PTGI } \\
\text { scores and greater growth for } 3 \text { of } 5 \\
\text { subscales. } \\
\text { The LC group was more likely to } \\
\text { report PTG in the areas of social } \\
\text { relationships and appreciation for life. }\end{array}$ \\
\hline $\begin{array}{l}\text { Baník and } \\
\text { Gajdošová } \\
\text { [4] }\end{array}$ & $\begin{array}{l}72(35 \\
\text { men; } 37 \\
\text { women })\end{array}$ & $\begin{array}{l}\text { Hematolog } \\
\text { ical (Non- } \\
\text { Hodgkin } \\
\text { lymphoma, } \\
\text { Hodgkin } \\
\text { lymphoma, } \\
\text { myeloma, } \\
\text { leukemia) } \\
\text { (Stage nr) }\end{array}$ & $\begin{array}{l}6 \text { months- } \\
1 \text { year: } \\
N=19 \\
1-2 \text { years: } \\
N=10 \\
2-5 \text { years: } \\
N=15 \\
\text { Over } 5 \\
\text { years: } \\
N=28\end{array}$ & $\begin{array}{l}\text { Cross- } \\
\text { sectional }\end{array}$ & $\begin{array}{l}\text { PTGI } \\
\text { BFS } \\
-C^{110}\end{array}$ & $\mathrm{nr}$ & $\begin{array}{l}\text { Age, type of cancer, } \\
\text { tolerance to physical } \\
\text { distress, positive } \\
\text { findings, hope, } \\
\text { optimism }\end{array}$ & $\begin{array}{l}\text { DTS }^{2} \\
\text { AHTS }^{3} \\
\text { LOT-R }^{4}\end{array}$ & $\begin{array}{l}\text { Higher perceptions of BF and greater } \\
\text { effort to regulate feelings of distress } \\
\text { predict PTG. }\end{array}$ \\
\hline $\begin{array}{l}\text { Bellizzi and } \\
\text { Blank [5] }\end{array}$ & $\begin{array}{l}224 \\
\text { women }\end{array}$ & $\begin{array}{l}\text { Breast } \\
\text { (Stage I- } \\
\text { IV) }\end{array}$ & $\begin{array}{l}\text { Range = } 1 \\
\text { year out } \\
\text { from } \\
\text { treatment } \\
\text { to } 4 \text { years } \\
\text { posttreat } \\
\text { ment }\end{array}$ & $\begin{array}{l}\text { Cross- } \\
\text { sectional }\end{array}$ & PTGI & $\mathrm{nr}$ & $\begin{array}{l}\text { Age, education, } \\
\text { employment, } \\
\text { children, children at } \\
\text { home, ethnicity, prior } \\
\text { health, time since } \\
\text { diagnosis, type of } \\
\text { cancer, treatment, } \\
\text { optimism, hope, } \\
\text { coping, intensity- } \\
\text { impact of cancer } \\
\end{array}$ & $\begin{array}{l}\text { LOT-R } \\
\text { HS }^{5} \\
\text { Brief COPE }^{6}\end{array}$ & $\begin{array}{l}\text { Age, marital status, employment, } \\
\text { education, perceived intensity of } \\
\text { disease, and active coping were the } \\
\text { main predictors of PTG. }\end{array}$ \\
\hline $\begin{array}{l}\text { Bellizzi et } \\
\text { al. [6] }\end{array}$ & $\begin{array}{l}802 \\
\text { women }\end{array}$ & $\begin{array}{l}\text { Breast } \\
\text { (Stage I- } \\
\text { IIIA) }\end{array}$ & $\begin{array}{l}M=6.1 \\
\text { months }\end{array}$ & $\begin{array}{l}\text { Cross- } \\
\text { sectional }\end{array}$ & PTGI & $\begin{array}{l}M=47.4 \\
S D=28.1\end{array}$ & $\begin{array}{l}\text { Age, employment, } \\
\text { race, optimism, } \\
\text { religiosity, time since } \\
\text { diagnosis, stage of } \\
\text { disease, health- } \\
\text { related quality of life, } \\
\text { optimism, religiosity } \\
\end{array}$ & $\begin{array}{l}\text { SF-36 } \\
\text { LOT-R } \\
\text { DRI }^{8}\end{array}$ & $\begin{array}{l}\text { Non-significant differences were found } \\
\text { between ethnicity and PTG. } \\
\text { PTG was inversely associated with } \\
\text { HRQOL. } \\
\text { Older age, being unemployed, lower } \\
\text { disease stage was associated with } \\
\text { lower PTG. }\end{array}$ \\
\hline $\begin{array}{l}\text { Bozo et al. } \\
\text { [7] }\end{array}$ & $\begin{array}{l}104 \\
\text { women }\end{array}$ & $\begin{array}{l}\text { Breast } \\
\text { (Stage I- } \\
\text { IV) }\end{array}$ & $\begin{array}{l}M=29.15 \\
\text { months } \\
S D= \\
49.88 \\
\text { months }\end{array}$ & $\begin{array}{l}\text { Cross- } \\
\text { sectional }\end{array}$ & PTGI & $\begin{array}{l}M=21.39 \\
S D=7.54\end{array}$ & $\begin{array}{l}\text { Optimism, perceived } \\
\text { social support }\end{array}$ & $\begin{array}{l}\text { LOT-R } \\
\text { MSPSS }^{9}\end{array}$ & $\begin{array}{l}\text { Higher dispositional optimism and } \\
\text { greater social support were significant } \\
\text { predictors of PTG. }\end{array}$ \\
\hline $\begin{array}{l}\text { Brix et al. } \\
{[8]}\end{array}$ & $\begin{array}{l}1350 \\
(684 \\
\text { women; } \\
666 \\
\text { healthy } \\
\text { control) }\end{array}$ & $\begin{array}{l}\text { Breast } \\
\text { (Stage 0- } \\
\text { III) }\end{array}$ & $\begin{array}{l}M=6.9 \\
\text { years } \\
\text { Range } 0.5 \\
\text { to } 15.5 \\
\text { years }\end{array}$ & $\begin{array}{l}\text { Longitudinal } \\
\text { T1 = Baseline; } \\
\text { T2 = 13-17 } \\
\text { years after } \mathrm{T} 1\end{array}$ & PTGI & $\begin{array}{l}M=36.0 \\
S D=34.2\end{array}$ & $\begin{array}{l}\text { Age, education, time } \\
\text { since operation, } \\
\text { tumor size, number } \\
\text { of positive lymph } \\
\text { nodes, type of } \\
\text { surgery, } \\
\text { chemotherapy, } \\
\text { radiotherapy, } \\
\text { endocrine treatment }\end{array}$ & & $\begin{array}{l}\text { No statistically significant difference in } \\
\text { overall PTG is observed between BC } \\
\text { women and BC-free women. } \\
\text { Among women with BC, time since } \\
\text { operation, tumour size, number of } \\
\text { positive lymph nodes, mastectomy } \\
\text { were positively associated with overall } \\
\text { PTG. Age was negatively associated } \\
\text { with PTG. }\end{array}$ \\
\hline
\end{tabular}


PTG IN ADULT CANCER PATIENTS: SYSTEMATIC REVIEW

\begin{tabular}{|c|c|c|c|c|c|c|c|c|c|}
\hline $\begin{array}{l}\text { Bussell and } \\
\text { Naus [9] }\end{array}$ & \begin{tabular}{|l|}
24 \\
women
\end{tabular} & $\begin{array}{l}\text { Breast } \\
\text { (Stage nr) }\end{array}$ & $\mathrm{Nr}$ & $\begin{array}{l}\text { Longitudinal } \\
\text { T1 = } \\
\text { undergoing } \\
\text { chemotherapy; } \\
\text { T2 = 2 year } \\
\text { after T1 }\end{array}$ & $\begin{array}{l}\text { PTGI } \\
(\text { at } \\
\text { T2) }\end{array}$ & $\mathrm{Nr}$ & $\begin{array}{l}\text { Coping, distressed } \\
\text { mood, depression, } \\
\text { anxiety, fatigue, } \\
\text { symptoms, perceived } \\
\text { stress }\end{array}$ & $\begin{array}{l}\text { Brief COPE } \\
\text { PSS }^{10} \\
\text { BFI }^{11} \\
\text { BAI }^{12} \\
\text { BDI }^{13} \\
\text { POMS }^{14}\end{array}$ & $\begin{array}{l}\text { Use of religion (T1) predicts PTG (T2) } \\
\text { At T2, using religion, positive } \\
\text { reframing, and acceptance predicts } \\
\text { PTG (T2). Instrumental and emotional } \\
\text { support are associated to higher PTG. } \\
\text { Lower perceived stress is marginally } \\
\text { related to PTG. }\end{array}$ \\
\hline $\begin{array}{l}\text { Büyükaşik- } \\
\text { Çolak et al. } \\
{[10]}\end{array}$ & $\begin{array}{l}90 \\
\text { women }\end{array}$ & $\begin{array}{l}\text { Breast } \\
\text { (Stage I- } \\
\text { IV) }\end{array}$ & $\begin{array}{l}M=12.54 \\
\text { months } \\
\text { Range }= \\
2-60 \\
\text { months }\end{array}$ & $\begin{array}{l}\text { Cross- } \\
\text { sectional }\end{array}$ & PTGI & $\mathrm{Nr}$ & Optimism, coping & $\begin{array}{l}\text { LOT-R } \\
\text { WCI }^{15}\end{array}$ & $\begin{array}{l}\text { Problem-focused coping and emotion- } \\
\text { focused coping were significant } \\
\text { predictors of PTG. }\end{array}$ \\
\hline $\begin{array}{l}\text { Cavell et al. } \\
{[12]}\end{array}$ & $\begin{array}{l}92(68 \\
\text { men; } 24 \\
\text { women })\end{array}$ & $\begin{array}{l}\text { Neck and } \\
\text { head; } \\
\text { Larynx; } \\
\text { Pharynx; } \\
\text { Oral; } \\
\text { Other. } \\
\text { (Stage I- } \\
\text { IV) }\end{array}$ & $\begin{array}{l}\text { Range = } \\
12-18 \\
\text { months }\end{array}$ & $\begin{array}{l}\text { Cross- } \\
\text { sectional }\end{array}$ & BFS & $\begin{array}{l}M=52.13 \\
S D=19.06\end{array}$ & $\begin{array}{l}\text { Age, gender, } \\
\text { ethnicity, disease } \\
\text { stage, treatment, site } \\
\text { of primary disease, } \\
\text { time since diagnosis, } \\
\text { baseline unmet } \\
\text { needs, quality of life, } \\
\text { depression, anxiety, } \\
\text { fear of recurrence, } \\
\text { coping }\end{array}$ & $\begin{array}{l}\text { HNCQOLS }^{16} \\
\text { PHQ }^{17} \\
\text { Brief STAI }^{18} \\
\text { Brief COPE }\end{array}$ & $\begin{array}{l}\text { European ethnicity, Maori/Pacific } \\
\text { Island ethnicity and active coping } \\
\text { strategies were significant predictors of } \\
\text { BF. }\end{array}$ \\
\hline $\begin{array}{l}\text { Chan et al. } \\
{[13]}\end{array}$ & $\begin{array}{l}170 \\
\text { women }\end{array}$ & $\begin{array}{l}\text { Breast } \\
\text { (Stage 0- } \\
\text { III) }\end{array}$ & $\begin{array}{l}M=15.59 \\
\text { months } \\
S D=5.84 \\
\text { months }\end{array}$ & $\begin{array}{l}\text { Cross- } \\
\text { sectional }\end{array}$ & PTGI & $\mathrm{nr}$ & $\begin{array}{l}\text { Positive and negative } \\
\text { attention bias, } \\
\text { rumination, PTSD } \\
\text { symptoms }\end{array}$ & $\begin{array}{l}\text { APNIS }^{19} \\
\text { CRRS }^{20} \\
\text { IES-R }\end{array}$ & $\begin{array}{l}\text { Positive attentional bias and positive } \\
\text { cancer-related rumination were } \\
\text { positively related to PTG. } \\
\text { Negative attentional bias and negative } \\
\text { cancer-related rumination were not } \\
\text { related to PTG. }\end{array}$ \\
\hline $\begin{array}{l}\text { Cohen and } \\
\text { Numa [14] }\end{array}$ & \begin{tabular}{|l}
124 \\
women \\
$(84$ \\
volunte \\
ers; 40 \\
non- \\
volunte \\
ers)
\end{tabular} & $\begin{array}{l}\text { Breast } \\
\text { (Stage nr) }\end{array}$ & $\begin{array}{l}\text { Volunteer } \\
\text { s }(M= \\
12.5 \\
\text { years; } S D \\
=7.3 \\
\text { years }) \\
\text { Non- } \\
\text { volunteers } \\
(M=7.4 \\
\text { years; } S D \\
=5 \text { years }) \\
\\
\text { Range }=7 \\
\text { to } 12 \\
\text { years }\end{array}$ & $\begin{array}{l}\text { Cross- } \\
\text { sectional }\end{array}$ & PTGI & $\begin{array}{l}\text { Volunteers } \\
(M=69.86 \\
S D=19.73) \\
\text { Non- } \\
\text { volunteers } \\
(M=70.72 \\
S D=15.02)\end{array}$ & $\begin{array}{lr}\text { Education, years } \\
\text { since diagnosis, type } \\
\text { of surgery, self- } \\
\text { reported health, } \\
\text { emotional expression } \\
\text { and emotional } \\
\text { processing, cognitive } \\
\text { processing, } \\
\text { perceived social } \\
\text { support }\end{array}$ & $\begin{array}{l}\text { EEPS }^{22} \\
\text { CPS }^{23} \\
\text { MSPSS }\end{array}$ & $\begin{array}{l}\text { Participants in both groups reported } \\
\text { similar and relatively high levels of } \\
\text { PTG. } \\
\text { PTG was not associated with } \\
\text { education, years since diagnosis, and } \\
\text { type of surgery. } \\
\text { PTG was positively associated with: } \\
\text { emotional processing, cognitive } \\
\text { processing, and social support. } \\
\text { In comparison with volunteer group, } \\
\text { in the non-volunteer group, a high and } \\
\text { significant correlation between self- } \\
\text { reported health and PTG. }\end{array}$ \\
\hline $\begin{array}{l}\text { Cordova et } \\
\text { al. [15] }\end{array}$ & \begin{tabular}{|l}
65 \\
women
\end{tabular} & $\begin{array}{l}\text { Breast } \\
\text { (Stage I-II) }\end{array}$ & $\begin{array}{l}M=9.4 \\
\text { months } \\
S D=6.4 \\
\text { months }\end{array}$ & $\begin{array}{l}\text { Cross- } \\
\text { sectional }\end{array}$ & PTGI & $\begin{array}{l}M=57.8 \\
S D=25.4\end{array}$ & $\begin{array}{l}\text { Age, education, } \\
\text { surgery, social } \\
\text { constraints, PTSD } \\
\text { symptoms, } \\
\text { stressfulness of the } \\
\text { cancer experience }\end{array}$ & $\begin{array}{l}\text { PCL-C }^{24} \\
\mathrm{SCS}^{25}\end{array}$ & $\begin{array}{l}\text { Younger age, higher education and } \\
\text { perception of cancer as a traumatic } \\
\text { event predict PTG. }\end{array}$ \\
\hline $\begin{array}{l}\text { Cormio et } \\
\text { al. [16] }\end{array}$ & $\begin{array}{l}360(57 \\
\text { men; } \\
303 \\
\text { women })\end{array}$ & $\begin{array}{l}\text { Breast; } \\
\text { Colorectal; } \\
\text { Lymphoma } \\
\text { s; Genital; } \\
\text { Others } \\
\text { (Stage nr) }\end{array}$ & $\begin{array}{l}M=11 \\
\text { years of } \\
\text { survival } \\
\text { Range = } \\
5-32 \\
\text { months }\end{array}$ & $\begin{array}{l}\text { Cross- } \\
\text { sectional }\end{array}$ & PTGI & $\begin{array}{l}M=36.63 \\
S D=25.64\end{array}$ & $\begin{array}{l}\text { Age, comorbidities, } \\
\text { physical activity, } \\
\text { coping, perceived } \\
\text { social support, health } \\
\text { behaviours }\end{array}$ & $\begin{array}{l}\text { Brief COPE } \\
\text { MSPSS }\end{array}$ & $\begin{array}{l}\text { Predictors of PTG (positive } \\
\text { association) were: physical activity, } \\
\text { social support from family and friends, } \\
\text { positive reframing, humour and } \\
\text { religious coping. Age and } \\
\text { comorbidities were negative significant } \\
\text { predictors of PTG. }\end{array}$ \\
\hline $\begin{array}{l}\text { Crawford et } \\
\text { al. [17] }\end{array}$ & $\begin{array}{l}621 \\
\text { women }\end{array}$ & $\begin{array}{l}\text { Gynaecolo } \\
\text { gical } \\
\text { (Ovarian, } \\
\text { endometria } \\
\text { 1, cervical) } \\
\text { (Stage nr) }\end{array}$ & $\begin{array}{l}M=118 \\
\text { months } \\
S D=77 \\
\text { months }\end{array}$ & $\begin{array}{l}\text { Cross- } \\
\text { sectional }\end{array}$ & $\begin{array}{l}\text { PTGI } \\
\text { BFS }\end{array}$ & $\mathrm{Nr}$ & $\begin{array}{l}\text { Exercise, impact of } \\
\text { cancer, benefit } \\
\text { finding }\end{array}$ & $\begin{array}{l}\text { IOC }^{26} \\
\text { GLTEQ }^{27} \\
\text { Strength } \\
\text { exercise }\end{array}$ & $\begin{array}{l}\text { Aerobic exercise and combined } \\
\text { (strength and aerobic) were positively } \\
\text { associated with the PTG. }\end{array}$ \\
\hline $\begin{array}{l}\text { Danhauer et } \\
\text { al. [18] }\end{array}$ & $\begin{array}{l}544 \\
\text { women }\end{array}$ & $\begin{array}{l}\text { Breast } \\
\text { (Stage I- } \\
\text { III) }\end{array}$ & $\begin{array}{l}\text { Median = } \\
4.37 \\
\text { months } \\
\text { Range - } \\
0.1 \text { to } 7.3 \\
\text { months }\end{array}$ & $\begin{array}{l}\text { Longitudinal } \\
\text { T1 = } 8 \text { months } \\
\text { of diagnosis; } \\
\text { T2 = } 6 \\
\text { months; } \mathrm{T} 3= \\
12 \text { months; } 44 \\
=18 \text { months } \\
\text { after } \mathrm{T} 1 \\
\end{array}$ & PTGI & $\mathrm{nr}$ & $\begin{array}{l}\text { Age, education, stage } \\
\text { of cancer, social } \\
\text { support, spirituality, } \\
\text { coping, general } \\
\text { health-related quality } \\
\text { of life, optimism, } \\
\text { illness intrusiveness }\end{array}$ & $\begin{array}{l}\text { RAND }^{28} \\
\text { FACIT- } \\
\text { Spiritual } \\
\text { Well-being } \\
\text { Brief COPE } \\
\text { SF-36 } \\
\text { LOT } \\
\text { IIRS }^{30}\end{array}$ & $\begin{array}{l}\text { Higher PTG was associated with } \\
\text { education level, longer time since } \\
\text { diagnosis, greater baseline level of } \\
\text { illness intrusiveness, increases in social } \\
\text { support, spirituality, use of active- } \\
\text { adaptive coping strategies, and mental } \\
\text { health. }\end{array}$ \\
\hline $\begin{array}{l}\text { Danhauer et } \\
\text { al. [19] }\end{array}$ & 37 & $\begin{array}{l}\text { Leukemia } \\
\text { (Stage nr) }\end{array}$ & $\mathrm{nr}$ & $\begin{array}{l}\text { Longitudinal } \\
\text { T1 = week } 0 \\
\text { or within } 7 \\
\text { days of } \\
\text { diagnosis } \\
\text { and/or } \\
\text { admission; T2 }\end{array}$ & PTGI & $\begin{array}{l}\text { T1 }(M=53.2 ; \\
S D=26.8) \\
\text { T2 }(M= \\
66.3 ; S D= \\
22.5) ; \mathrm{T} 3(M \\
=73.1 ; S D= \\
20.4)\end{array}$ & $\begin{array}{l}\text { Current mood, } \\
\text { severity and impact } \\
\text { of cancer-related } \\
\text { symptoms, sleep } \\
\text { quality, spiritual } \\
\text { well-being, social } \\
\text { constraints, } \\
\text { rumination, }\end{array}$ & $\begin{array}{l}\text { POMS-SF } \\
\text { MDASI }^{31} \\
\text { WHIRS }^{32} \\
\text { FACIT-Sp } \\
\text { SCS } \\
\text { CBI }^{33}\end{array}$ & $\begin{array}{l}\text { Greater number of days from baseline, } \\
\text { younger age, greater deliberate } \\
\text { rumination and greater challenge to } \\
\text { core beliefs were associated with } \\
\text { greater PTG over time. }\end{array}$ \\
\hline
\end{tabular}




\begin{tabular}{|c|c|c|c|c|c|c|c|c|c|}
\hline & & & & $\begin{array}{l}=\text { weeks } 5-6 \\
\text { or prior to } \\
\text { discharge from } \\
\text { the hospital if } \\
\text { patient was } \\
\text { discharged } \\
\text { prior to week } \\
5 ; \text { T3 = } \\
\text { approximately } \\
\text { weeks 9-13 } \\
\text { upon } \\
\text { readmission } \\
\text { for } \\
\text { consolidation } \\
\text { chemotherapy }\end{array}$ & & & $\begin{array}{l}\text { perceived threat, core } \\
\text { beliefs }\end{array}$ & & \\
\hline $\begin{array}{l}\text { Danhauer et } \\
\text { al. [20] }\end{array}$ & $\begin{array}{l}653 \\
\text { women }\end{array}$ & $\begin{array}{l}\text { Breast } \\
\text { (Stage I- } \\
\text { III) }\end{array}$ & $\begin{array}{l}\text { Median = } \\
4.7 \\
\text { months; } \\
\text { Range }= \\
.1-7.3\end{array}$ & $\begin{array}{l}\text { Longitudinal } \\
\text { T1-within } 8 \\
\text { months after } \\
\text { diagnosis; T2 - } \\
6 \text { months; T3 - } \\
12 \text { months; } \\
\text { T4-18 months } \\
\text { after T1 }\end{array}$ & PTGI & $\begin{array}{l}\text { T1 }(M= \\
54.0 ; S D= \\
23.2) \\
\text { T2 }(M= \\
56.77 ; S D= \\
23.1) \\
\text { T3 }(M= \\
57.1 ; S D= \\
22.9) \\
\text { T4 }(M= \\
58.4 ; S D= \\
22.8)\end{array}$ & $\begin{array}{l}\text { Race, marital status, } \\
\text { education, cancer } \\
\text { stage, surgery, age, } \\
\text { depressive } \\
\text { symptoms, coping } \\
\text { strategies, illness } \\
\text { intrusiveness, } \\
\text { social support }\end{array}$ & $\begin{array}{l}\text { BDI } \\
\text { Brief COPE } \\
\text { IIRS } \\
\text { RAND }\end{array}$ & $\begin{array}{l}\text { Differences among trajectory groups } \\
\text { were significantly associated with age, } \\
\text { race, chemotherapy, illness } \\
\text { intrusiveness, depressive symptoms, } \\
\text { active-adaptive coping, and social } \\
\text { support. } \\
\text { Trajectory groups did not differ } \\
\text { significantly by marital status, } \\
\text { education, cancer stage, and passive } \\
\text { coping strategies. } \\
\text { In three trajectories PTG was stable } \\
\text { over time. The groups of low and } \\
\text { moderate PTG increased over the } 2 \\
\text { years and one trajectory increased } \\
\text { considerably PTG. }\end{array}$ \\
\hline $\begin{array}{l}\text { Diaz et al. } \\
{[21]}\end{array}$ & $\begin{array}{l}99 \\
\text { women }\end{array}$ & $\begin{array}{l}\text { Breast } \\
\text { (Stage IV) }\end{array}$ & $\begin{array}{l}25 \%=30 \\
\text { months; } \\
50 \%=77 \\
\text { months; } \\
75 \%= \\
116 \\
\text { months }\end{array}$ & $\begin{array}{l}\text { Cross- } \\
\text { sectional }\end{array}$ & PTGI & $\mathrm{nr}$ & Cortisol & $\begin{array}{l}\text { Saliva } \\
\text { samples }\end{array}$ & $\begin{array}{l}\text { There was a significant correlation } \\
\text { between PTG and diurnal cortisol } \\
\text { slope. }\end{array}$ \\
\hline $\begin{array}{l}\text { Dunn et al. } \\
\text { [22] }\end{array}$ & $\begin{array}{l}439 \\
(121 \\
\text { men; } \\
318 \\
\text { women) }\end{array}$ & $\begin{array}{l}\text { Breast, } \\
\text { haematolo } \\
\text { gical, skin, } \\
\text { gastrointest } \\
\text { inal, head } \\
\text { and neck, } \\
\text { respiratory, } \\
\text { genitourina } \\
\text { ry, other } \\
\text { (Stage nr) }\end{array}$ & $\begin{array}{l}M=87.5 \\
\text { weeks } \\
S D=97.4 \\
\text { weeks }\end{array}$ & $\begin{array}{l}\text { Cross- } \\
\text { sectional }\end{array}$ & BFS & $\mathrm{Nr}$ & $\begin{array}{l}\text { Age, marital status, } \\
\text { gender, time since } \\
\text { diagnosis, education, } \\
\text { public/private, } \\
\text { anxiety and } \\
\text { depression, quality of } \\
\text { life, intrusion and } \\
\begin{array}{lr}\text { avoidance, social } \\
\text { support, social } \\
\text { constraints }\end{array}\end{array}$ & $\begin{array}{l}\text { HADS } \\
\text { SF-36 } \\
\text { ENRICHD } \\
\text { LOT-R } \\
\text { SCS } \\
\text { IES-R }\end{array}$ & $\begin{array}{l}\text { BF was predicted by female gender, } \\
\text { greater optimism, high intrusive } \\
\text { thinking, high social support and social } \\
\text { constraints. } \\
\text { BF was marginally related to } \\
\text { depression. }\end{array}$ \\
\hline $\begin{array}{l}\text { Garland et } \\
\text { al. [23] }\end{array}$ & $\begin{array}{l}316 \\
(112 \\
\text { men; } \\
204 \\
\text { women }) \\
\\
(193 \\
\text { CAM } \\
\text { use, } 122 \\
\text { No } \\
\text { CAM } \\
\text { use })\end{array}$ & $\begin{array}{l}\text { Breast, } \\
\text { Lung, } \\
\text { Gastrointes } \\
\text { tinal, other } \\
\text { (Stage nr) }\end{array}$ & $\begin{array}{l}\leq 12 \\
\text { months } \\
(141) \\
\geq 12 \text { to } 36 \\
\text { months } \\
(80) \\
>36 \\
\text { months } \\
(89)\end{array}$ & $\begin{array}{l}\text { Cross- } \\
\text { sectional }\end{array}$ & BFS & & $\begin{array}{l}\text { Use of } \\
\text { Complementary and } \\
\text { Alternative Medicine } \\
\text { (CAM), age, gender, } \\
\text { race/ ethnicity, } \\
\text { education, } \\
\text { employment, cancer } \\
\text { type, cancer stage, } \\
\text { surgery, radiation, } \\
\text { chemotherapy, time } \\
\text { since diagnosis }\end{array}$ & & $\begin{array}{l}\text { Race, time from diagnosis, age and } \\
\text { CAM use predicted BF. } \\
\text { Special diet, herbal remedies, vitamin } \\
\text { use, and massage saw a smaller } \\
\text { increase in BF, while acupuncture, } \\
\text { chiropractic, homeopathy, relaxation, } \\
\text { yoga, and tai chi were not significantly } \\
\text { associated with BF. }\end{array}$ \\
\hline $\begin{array}{l}\text { Harrington } \\
\text { et al. [25] }\end{array}$ & $\begin{array}{l}76(37 \\
\text { men; } \\
39 \\
\text { women })\end{array}$ & $\begin{array}{l}\text { Head and } \\
\text { neck } \\
\text { (Stage I- } \\
\text { IV) }\end{array}$ & $\begin{array}{l}\text { Range = } \\
0-24 \\
\text { months }\end{array}$ & $\begin{array}{l}\text { Cross- } \\
\text { sectional }\end{array}$ & BFS & $\begin{array}{l}M=3.55 \\
S D=.44\end{array}$ & $\begin{array}{l}\text { Type of treatment, } \\
\text { stage of cancer, type } \\
\text { of cancer, optimism, } \\
\text { coping, anxiety and } \\
\text { depression }\end{array}$ & $\begin{array}{l}\text { LOT-R } \\
\text { Brief COPE } \\
\text { HADS }\end{array}$ & $\begin{array}{l}\text { Optimism and positive reappraisal } \\
\text { were predictors of BF. }\end{array}$ \\
\hline $\begin{array}{l}\text { Heidarzade } \\
h \text { et al. [27] }\end{array}$ & $\begin{array}{l}452 \\
(185 \\
\text { men; } \\
267 \\
\text { women) }\end{array}$ & $\begin{array}{l}\text { Breast; } \\
\text { Gastrointes } \\
\text { tinal; } \\
\text { Hematolog } \\
\text { y; Prostate; } \\
\text { Lung; } \\
\text { Uterus and } \\
\text { ovaries; } \\
\text { Others } \\
\text { (Stage nr) }\end{array}$ & $\begin{array}{l}2 \text { years } \\
(71) \\
3 \text { years } \\
(34) \\
4 \text { years } \\
(20) \\
>5 \text { years } \\
(72) \\
\text { Range }=2 \\
\text { to 5 years }\end{array}$ & $\begin{array}{l}\text { Cross- } \\
\text { sectional }\end{array}$ & PTGI & $\begin{array}{l}M=68.6 \\
S D=14.6\end{array}$ & $\begin{array}{l}\text { Age, gender, } \\
\text { education, income, } \\
\text { marital status, } \\
\text { occupation, duration } \\
\text { of cancer diagnosis, } \\
\text { growth of cancer, } \\
\text { type of cancer }\end{array}$ & & $\begin{array}{l}\text { Age is negatively correlated with PTG } \\
\text { Educational status, income had a } \\
\text { positive and significant correlation } \\
\text { with PTG. }\end{array}$ \\
\hline $\begin{array}{l}\text { Ho et al. } \\
{[28]}\end{array}$ & $\begin{array}{l}90 \\
\text { women }\end{array}$ & $\begin{array}{l}\text { Breast } \\
\text { (Stage 0- } \\
\text { IV) }\end{array}$ & $\begin{array}{l}\text { Range = } \\
7-9 \\
\text { months }\end{array}$ & $\begin{array}{l}\text { Cross- } \\
\text { sectional }\end{array}$ & PTGI & $\mathrm{nr}$ & $\begin{array}{l}\text { Explanatory styles, } \\
\text { PTSD symptoms }\end{array}$ & $\begin{array}{l}\mathrm{ASCQ}^{35} \\
\text { IES-R }\end{array}$ & $\begin{array}{l}\text { Explanatory style for good events, but } \\
\text { not for bad events, was significantly } \\
\text { associated with PTG. } \\
\text { The tendency to globalise the causes of } \\
\text { good events was the most important }\end{array}$ \\
\hline
\end{tabular}


PTG IN ADULT CANCER PATIENTS: SYSTEMATIC REVIEW

\begin{tabular}{|c|c|c|c|c|c|c|c|c|c|}
\hline & & & & & & & & & predictors of PTG. \\
\hline $\begin{array}{l}\text { Ho et al. } \\
{[29]}\end{array}$ & $\begin{array}{l}50(21 \\
\text { men; } 29 \\
\text { women })\end{array}$ & $\begin{array}{l}\text { Oral cavity } \\
\text { (Stage } n r)\end{array}$ & NR & $\begin{array}{l}\text { Cross- } \\
\text { sectional }\end{array}$ & PTGI & $\begin{array}{l}M=51.76 \\
S D=11.18\end{array}$ & $\begin{array}{l}\text { Age, time since } \\
\text { diagnosis, religion, } \\
\text { gender, economical } \\
\text { status, education, } \\
\text { marital status, stag of } \\
\text { cancer, treatment, } \\
\text { hope, optimism } \\
\end{array}$ & $\begin{array}{l}\text { HS } \\
\text { LOT-R }\end{array}$ & $\begin{array}{l}\text { Hope and optimism are strong } \\
\text { predictors of PTG. } \\
\text { Greater PTG showed significant } \\
\text { relationship with higher income and } \\
\text { being married. A negative correlation } \\
\text { was found between stage of cancer and } \\
\text { PTG. }\end{array}$ \\
\hline $\begin{array}{l}\text { Kangas et } \\
\text { al. [31] }\end{array}$ & $\begin{array}{l}70(16 \\
\text { men; } 54 \\
\text { women }) \\
(27 \\
\text { early } \\
\text { subgrou } \\
\text { p; } 43 \\
\text { late } \\
\text { subgrou } \\
\text { p) }\end{array}$ & $\begin{array}{l}\text { Meningio } \\
\text { ma } \\
\text { (Stage nr) }\end{array}$ & $\begin{array}{l}\text { Early } \\
\text { subgroup: } \\
M=11.3 \\
\text { months; } \\
S D=6.3 \\
\text { months } \\
\text { Late } \\
\text { subgroup: } \\
M=79.9 \\
\text { months; } \\
S D=54.5 \\
\text { months }\end{array}$ & $\begin{array}{l}\text { Cross- } \\
\text { sectional }\end{array}$ & BFS & $\mathrm{nr}$ & $\begin{array}{l}\text { Emotional well- } \\
\text { being, intrusions and } \\
\text { avoidance, perceived } \\
\text { social support }\end{array}$ & $\begin{array}{l}\text { POMS } \\
\text { IES-R } \\
\text { MSPSS }\end{array}$ & $\begin{array}{l}\text { BF was significantly associated with } \\
\text { elevated depression symptoms (early } \\
\text { subgroup only). } \\
\text { BF was positively correlated with } \\
\text { intrusions and avoidance symptoms } \\
\text { (late subgroup only). }\end{array}$ \\
\hline $\begin{array}{l}\text { Karanci and } \\
\text { Erkam [32] }\end{array}$ & $\begin{array}{l}90 \\
\text { women }\end{array}$ & $\begin{array}{l}\text { Breast } \\
\text { (Stage I- } \\
\text { IV) }\end{array}$ & $\begin{array}{l}\text { Range }= \\
\text { 1month to } \\
8 \text { years }\end{array}$ & $\begin{array}{l}\text { Cross- } \\
\text { sectional }\end{array}$ & $\begin{array}{l}\text { SRG } \\
\text { S }\end{array}$ & $\begin{array}{l}M=143 \\
S D=12.32\end{array}$ & $\begin{array}{l}\text { Perceived social } \\
\text { support, coping, } \\
\text { depression }\end{array}$ & $\begin{array}{l}\text { MSPSS } \\
\text { WCI } \\
\text { BDI }\end{array}$ & $\begin{array}{l}\text { Social support, problem-solving coping } \\
\text { (positive association), income level and } \\
\text { depression (negative association) were } \\
\text { significant predictors of stress-related } \\
\text { growth. }\end{array}$ \\
\hline $\begin{array}{l}\text { Kent et al. } \\
{[33]}\end{array}$ & $\begin{array}{l}604 \\
\text { women }\end{array}$ & $\begin{array}{l}\text { Breast } \\
\text { (Stage I- } \\
\text { IIIA) }\end{array}$ & $\mathrm{nr}$ & $\begin{array}{l}\text { Longitudinal } \\
\text { T1 = 2-12 } \\
\text { months after } \\
\text { diagnosis; T2 } \\
=30 \text { months; } \\
\text { T3 = } 39 \\
\text { months after } \\
\text { diagnosis } \\
\end{array}$ & $\begin{array}{l}\text { PTGI } \\
\text { (at } \\
\text { T3) }\end{array}$ & $\begin{array}{l}M=48.8 \\
S D=27.4\end{array}$ & $\begin{array}{l}\text { Support seeking, } \\
\text { cancer-related } \\
\text { support programs, } \\
\text { religiosity }\end{array}$ & DRI & $\begin{array}{l}\text { Race/ethnicity, age, stage at diagnosis } \\
\text { (in situ/ localized/ regional), } \\
\text { religiosity, support program } \\
\text { participation, and confiding in a health } \\
\text { care provider were all significantly } \\
\text { associated with PTG. } \\
\text { A negative correlation between PTG } \\
\text { and age was found. }\end{array}$ \\
\hline $\begin{array}{l}\text { Kinsinger et } \\
\text { al. [34] }\end{array}$ & $\begin{array}{l}250 \\
\text { men }\end{array}$ & $\begin{array}{l}\text { Prostate } \\
\text { (Stage I-II) }\end{array}$ & $\begin{array}{l}M=15.7 \\
\text { months } \\
S D=6.9 \\
\text { months }\end{array}$ & $\begin{array}{l}\text { Cross- } \\
\text { sectional }\end{array}$ & BFS & $\mathrm{Nr}$ & $\begin{array}{l}\text { Age, education, } \\
\text { income, ethnicity, } \\
\text { months since } \\
\text { diagnosis, months } \\
\text { since treatment, } \\
\text { surgery vs. radiation, } \\
\text { medical } \\
\text { comorbidities, } \\
\text { quality of life, social } \\
\text { support, coping } \\
\end{array}$ & $\begin{array}{l}\mathrm{CCI}^{36} \\
\text { FACT-G }^{37} \\
\text { PCa index } \\
\text { composite }^{38} \\
\text { UCLA-PCI } \\
\text { Brief COPE } \\
\text { ENRICHD }\end{array}$ & $\begin{array}{l}\text { Active coping and social support were } \\
\text { significant predictors of } \mathrm{BF} \text {. }\end{array}$ \\
\hline $\begin{array}{l}\text { Lechner et } \\
\text { al. [36] }\end{array}$ & $\begin{array}{l}\frac{\text { Study } 1}{n=230} \\
\text { and } \\
\text { Long- } \\
\text { Term } \\
\text { Follow- } \\
\text { Up ( } N= \\
96) \\
\text { Study } 2 \\
n=136 \\
\text { Particip } \\
\text { ants } \\
\text { from a } \\
\text { stress } \\
\text { manage } \\
\text { ment } \\
\text { training }\end{array}$ & $\begin{array}{l}\text { Breast } \\
\text { (Stage 0-II) }\end{array}$ & $\mathrm{nr}$ & $\begin{array}{l}\text { Study } 1 \\
\text { Longitudinal } \\
(3,6, \text { or } 12 \\
\text { months } \\
\text { following } \\
\text { surgery and } \\
\text { then again at } \\
5-8 \text { years } \\
\text { postdiagnosis) } \\
\text { Study } 2 \\
\text { Longitudinal } \\
\text { (T1 = about } 2 \\
\text { months after } \\
\text { surgery; T2 = } \\
\text { postinterventio } \\
\text { n,5 months } \\
\text { postsurgery; } \\
\text { T3 = } 3 \\
\text { months } \\
\text { postinterventio } \\
\text { n, } 8 \text { months } \\
\text { postsurgery; } \\
\text { T4 = 9 months } \\
\text { postinterventio } \\
\text { n, } 14 \text { months } \\
\text { postsurgery. }\end{array}$ & BFS & $\begin{array}{l}\text { T1 }(M= \\
2.15 ; S D= \\
.63) \text { Follow- } \\
\text { up }(M= \\
2.33 ; S D= \\
.93)\end{array}$ & $\begin{array}{l}\text { Study } 1 \\
\text { Perceived quality of } \\
\text { life, distress, } \\
\text { depressive } \\
\text { symptoms, disruption } \\
\text { of social and } \\
\text { recreational } \\
\text { activities, optimism, } \\
\text { investment in body } \\
\text { image, coping } \\
\text { Study } 2 \\
\text { Perceived quality of } \\
\text { life, depressive } \\
\text { symptoms, disruption } \\
\text { of social and } \\
\text { recreational } \\
\text { activities, positive } \\
\text { and negative affect }\end{array}$ & $\begin{array}{l}\frac{\text { Study } 1}{\text { PQOL }^{40}} \\
\text { CES-D } \\
\text { SIP }^{42} \\
\text { LOT-R }^{41} \\
\text { MBA }^{43} \\
\text { Brief COPE } \\
\text { Study } 2 \\
\text { PQOL } \\
\text { CES-D } \\
\text { SIP } \\
\text { ABS }\end{array}$ & $\begin{array}{l}\text { Women with low or high BF had better } \\
\text { adjustment than those with } \\
\text { intermediate BF. } \\
\text { Long-term BF was associated with } \\
\text { QoL, positive affect, negative affect } \\
\text { and social disruption. There was found } \\
\text { a quadratic relationship between BF } \\
\text { and QoL.(Study 1) } \\
\text { Quadratic relationships were found } \\
\text { between BF and social disruption, } \\
\text { avoidance, intrusion, negative emotion, } \\
\text { QoL and positive affect. BF was } \\
\text { associated with long-term positive } \\
\text { affect (Study 2). }\end{array}$ \\
\hline $\begin{array}{l}\text { Lelorain et } \\
\text { al. [37] }\end{array}$ & $\begin{array}{l}307 \\
\text { women }\end{array}$ & $\begin{array}{l}\text { Breast } \\
\text { (Stage I- } \\
\text { III) }\end{array}$ & $\begin{array}{l}M=10 \\
\text { years } \\
S D=2.8 \\
\text { years }\end{array}$ & $\begin{array}{l}\text { Cross- } \\
\text { sectional }\end{array}$ & PTGI & $\begin{array}{l}M=59.9 \\
S D=20\end{array}$ & $\begin{array}{l}\text { Chemotherapy, } \\
\text { mental health, } \\
\text { coping strategies, } \\
\text { positive affectivity, } \\
\text { long- term perceived } \\
\text { sequelae of cancer }\end{array}$ & $\begin{array}{l}\text { SF-36 } \\
\text { Brief COPE } \\
\text { PANAS }\end{array}$ & $\begin{array}{l}\text { Dispositional positive affectivity and } \\
\text { adaptive coping of positive, active, } \\
\text { relational, religious and denial coping } \\
\text { have a strong effect on PTG. } \\
\text { PTG is associated with mental QoL } \\
\text { and happiness. Perceived 'somewhat }\end{array}$ \\
\hline
\end{tabular}




\begin{tabular}{|c|c|c|c|c|c|c|c|c|c|}
\hline & & & & & & & and treatment & & $\begin{array}{l}\text { troublesome sequelae' was positively } \\
\text { associated with PTG whereas 'very } \\
\text { troublesome sequelae' were inversely } \\
\text { associated with PTG. }\end{array}$ \\
\hline $\begin{array}{l}\text { Li et al. } \\
{[38]}\end{array}$ & $\begin{array}{l}200(95 \\
\text { men; } \\
105 \\
\text { women })\end{array}$ & $\begin{array}{l}\text { Lung; } \\
\text { Lymphoma } \\
\text { Leukemia } \\
\text { (Stage nr) }\end{array}$ & $\begin{array}{l}M=35 \\
\text { months } \\
S D= \\
44.43 \\
\text { months }\end{array}$ & $\begin{array}{l}\text { Cross- } \\
\text { sectional }\end{array}$ & $\begin{array}{l}\text { PTGI } \\
\text { BFS }\end{array}$ & $\begin{array}{l}M=37.14 \\
S D=18.44\end{array}$ & $\begin{array}{l}\text { Time since } \\
\text { diagnosis, type of } \\
\text { cancer, existential } \\
\text { distress, sense- } \\
\text { making, benefit } \\
\text { finding }\end{array}$ & $\mathrm{DS}^{46}$ & $\mathrm{BF}$ is the strong predictor of PTG \\
\hline $\begin{array}{l}\text { Liu et al. } \\
\text { [39] }\end{array}$ & $\begin{array}{l}120 \\
\text { women }\end{array}$ & $\begin{array}{l}\text { Breast } \\
\text { (Stage I-II) }\end{array}$ & $\mathrm{nr}$ & $\begin{array}{l}\text { Longitudinal } \\
\text { T1= } 3 \text { months } \\
\text { after } \\
\text { diagnosis; T2= } \\
6 \text { months; T3= } \\
9 \text { months after } \\
\text { T1 }\end{array}$ & PTGI & $\begin{array}{l}\mathrm{T} 1(M= \\
63.24 ; \mathrm{SD}= \\
14.21) ; \mathrm{T} 2 \\
(\mathrm{M}=68.26 \\
\mathrm{SD}=15.29) \\
\mathrm{T} 3(\mathrm{M}= \\
70.29 ; \mathrm{SD}= \\
16.07)\end{array}$ & $\begin{array}{l}\text { Distress, several } \\
\text { problems cancer- } \\
\text { related (physical, } \\
\text { practical, with } \\
\text { relatives, emotional, } \\
\text { philosophical) }\end{array}$ & $\begin{array}{l}\text { Distress } \\
\text { thermometer }\end{array}$ & $\begin{array}{l}\text { PTG showed a significant negative } \\
\text { correlation with psychological distress. }\end{array}$ \\
\hline $\begin{array}{l}\text { Llewellyn } \\
\text { et al. [40] }\end{array}$ & $\begin{array}{l}103(73 \\
\text { men; } 30 \\
\text { women })\end{array}$ & $\begin{array}{l}\text { Head and } \\
\text { neck } \\
\text { (Stage nr) }\end{array}$ & $\mathrm{nr}$ & $\begin{array}{l}\text { Longitudinal } \\
\text { T1 = period } \\
\text { between } \\
\text { confirmation } \\
\text { of diagnosis } \\
\text { and before } \\
\text { treatment } \\
\text { T2 = } 6 \text { months } \\
\text { after treatment } \\
\text { completion }\end{array}$ & BFS & $\begin{array}{l}\mathrm{T} 1(M= \\
59.79 ; S D= \\
7.59) ; \mathrm{T} 2(M \\
=58.13 ; S D \\
=8.22)\end{array}$ & $\begin{array}{l}\text { Marital status, level } \\
\text { of education, } \\
\text { distress, optimism, } \\
\text { coping, perceived } \\
\text { quality of life }\end{array}$ & $\begin{array}{l}\text { HADS } \\
\text { LOT-R } \\
\text { Brief COPE } \\
\text { SF-12v2 } \\
\text { EORTC } \\
\text { QLQ-C30 } 37\end{array}$ & $\begin{array}{l}\text { BF was predicted by active coping } \\
\text { strategies and use of emotional } \\
\text { support. } \\
\text { Optimism, living with a partner and } \\
\text { higher educational attainment were } \\
\text { also associated with higher BF and } \\
\text { have a protective effect. }\end{array}$ \\
\hline $\begin{array}{l}\text { Martins da } \\
\text { Silva et al. } \\
{[41]}\end{array}$ & $\begin{array}{l}160(71 \\
\text { breast } \\
\text { cancer } \\
\text { survivor } \\
\text { s (BC); } \\
89 \\
\text { health } \\
\text { controls } \\
(\text { HC) })\end{array}$ & $\begin{array}{l}\text { Breast } \\
\text { (Stage nr) }\end{array}$ & $\begin{array}{l}M=13.5 \\
\text { months } \\
S D=4.6 \\
\text { months }\end{array}$ & $\begin{array}{l}\text { Cross- } \\
\text { sectional }\end{array}$ & PTGI & $\begin{array}{l}\mathrm{BC}(M= \\
63.93 ; S D= \\
27.91) ; \mathrm{HC} \\
(M=40.63 ; \\
S D=26.56)\end{array}$ & $\begin{array}{l}\text { Emotional distress, } \\
\text { quality of life, } \\
\text { subjective perception } \\
\text { of breast cancer as a } \\
\text { traumatic experience }\end{array}$ & $\begin{array}{l}\text { HADS } \\
\text { WHOQOL - } \\
\text { BREF }^{48}\end{array}$ & $\begin{array}{l}\text { The BC survivor group showed greater } \\
\text { total PTG than the HC group. } \\
\text { PTG was negatively associated with } \\
\text { perception of trauma and depression. }\end{array}$ \\
\hline $\begin{array}{l}\text { McDonoug } \\
\text { h et al. [42] }\end{array}$ & $\begin{array}{l}173 \\
\text { women }\end{array}$ & $\begin{array}{l}\text { Breast } \\
\text { (Stage I- } \\
\text { III) }\end{array}$ & $\begin{array}{l}M=11.37 \\
\text { months } \\
S D=4.36 \\
\text { months }\end{array}$ & $\begin{array}{l}\text { Longitudinal } \\
\text { T1 = baseline; } \\
\text { T2 = 3-month; } \\
\text { T3 = 6-month } \\
\text { after T1 }\end{array}$ & $\begin{array}{l}\text { PTGI } \\
\text { (at } \\
\text { T2 } \\
\text { and } \\
\text { T3) }\end{array}$ & $\begin{array}{l}\mathrm{T} 2(M= \\
2.97 ; S D= \\
1.00) ; \mathrm{T} 3(M \\
=2.97 ; S D= \\
1.04)\end{array}$ & $\begin{array}{l}\text { Social support, } \\
\text { perceived stress, } \\
\text { cancer worry, } \\
\text { subjective well-being }\end{array}$ & $\begin{array}{l}\text { SSS }^{49} \\
\text { PSS } \\
\text { ASCQ } \\
\text { PWB }^{50}\end{array}$ & $\begin{array}{l}\text { BC-specific social support (T2) and } \\
\text { cancer worry (T2) were significant } \\
\text { predictors of PTG (T2). }\end{array}$ \\
\hline $\begin{array}{l}\text { Mols et al. } \\
{[43]}\end{array}$ & $\begin{array}{l}183 \\
\text { women }\end{array}$ & $\begin{array}{l}\text { Breast } \\
\text { (Stage I-II) }\end{array}$ & NR & $\begin{array}{l}\text { Cross- } \\
\text { sectional }\end{array}$ & $\begin{array}{l}\text { PTGI } \\
\text { BFS }\end{array}$ & $\begin{array}{l}\text { Mean of sub- } \\
\text { scales } \\
\text { between } \mathrm{M}= \\
2.8(\mathrm{SD}= \\
5.8) \text { to } \mathrm{M}= \\
19.9(\mathrm{SD}= \\
7.8)\end{array}$ & $\begin{array}{l}\text { Health status and } \\
\text { subjective well- } \\
\text { being, benefit finding }\end{array}$ & $\begin{array}{l}\text { PDIS }^{51} \\
\text { Center data } \\
\text { Health } \\
\text { monitor }\end{array}$ & $\begin{array}{l}\text { PTG showed a positive correlation } \\
\text { with BF, higher satisfaction with life. } \\
\text { PTG was negatively associated with } \\
\text { Radiotherapy. } \\
\text { Women with a higher tumour stage at } \\
\text { diagnosis experienced less BF in } \\
\text { comparison to women with a lower } \\
\text { tumour stage at diagnosis. }\end{array}$ \\
\hline $\begin{array}{l}\text { Morris and } \\
\text { Shakespear } \\
\text { e-Finch [44] }\end{array}$ & $\begin{array}{l}335 \\
(150 \\
\text { men; } \\
185 \\
\text { women })\end{array}$ & $\begin{array}{l}\text { Breast; } \\
\text { Prostate; } \\
\text { Haematolo } \\
\text { gical; } \\
\text { Colorectal } \\
\text { (Stage nr) }\end{array}$ & $\mathrm{nr}$ & $\begin{array}{l}\text { Cross- } \\
\text { sectional }\end{array}$ & PTGI & $\begin{array}{l}M=59.29 \\
S D=22.36\end{array}$ & 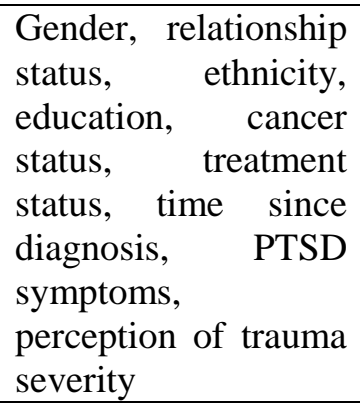 & IES-R & $\begin{array}{l}\text { PTG was significantly and positively } \\
\text { associated with type of cancer, trauma } \\
\text { severity and distress. } \\
\text { Breast cancer survivors reported } \\
\text { significantly higher levels of PTG than } \\
\text { those diagnosed with colorectal and } \\
\text { hematological malignancies, but not } \\
\text { significantly different from prostate } \\
\text { cancer survivors. }\end{array}$ \\
\hline $\begin{array}{l}\text { Morris and } \\
\text { Shakespear } \\
\text { e-Finch [45] }\end{array}$ & $\begin{array}{l}313 \\
(137 \\
\text { men; } \\
176 \\
\text { women) }\end{array}$ & $\begin{array}{l}\text { Breast; } \\
\text { Prostate; } \\
\text { Haematolo } \\
\text { gical; } \\
\text { Colorectal } \\
\text { (Stage nr) } \\
\end{array}$ & $\begin{array}{l}M=2.92 \\
\text { years } \\
S D=1.86 \\
\text { years }\end{array}$ & $\begin{array}{l}\text { Cross- } \\
\text { sectional }\end{array}$ & PTGI & $\begin{array}{l}M=59.29 \\
S D=22.36\end{array}$ & $\begin{array}{l}\text { Seeking social } \\
\text { support, rumination, } \\
\text { overall distress }\end{array}$ & $\begin{array}{l}\text { IES-R } \\
\mathrm{COPE}^{52} \\
\mathrm{RI}^{53}\end{array}$ & $\begin{array}{l}\text { Deliberately ruminating on benefits } \\
\text { and social support were positively } \\
\text { related to PTG. }\end{array}$ \\
\hline $\begin{array}{l}\text { Morris et al. } \\
{[46]}\end{array}$ & $\begin{array}{l}335 \\
(150 \\
\text { men; } \\
185 \\
\text { women })\end{array}$ & $\begin{array}{l}\text { Breast, } \\
\text { prostate, } \\
\text { haematolo } \\
\text { gical, } \\
\text { colorectal/ } \\
\text { rectal, } \\
\text { gynaecolog } \\
\text { ical, lung, } \\
\text { head/neck, } \\
\text { gastric } \\
\text { (Stage nr) }\end{array}$ & $\begin{array}{l}\text { Range }= \\
1.5 \text { to } 4 \\
\text { years } \\
\text { since } \\
\text { diagnosis }\end{array}$ & $\begin{array}{l}\text { Cross- } \\
\text { sectional }\end{array}$ & PTGI & $\begin{array}{l}M=59.29 \\
S D=22.36\end{array}$ & Coping & COPE & $\begin{array}{l}\text { Positive reframing is positively } \\
\text { correlated with all PTGI. Age is } \\
\text { negatively associated with PTG. } \\
\text { Focussing on venting emotions, social } \\
\text { support engagement, and active coping } \\
\text { are associated with two dimensions of } \\
\text { PTG (New Possibilities and Relating to } \\
\text { Others). }\end{array}$ \\
\hline
\end{tabular}


PTG IN ADULT CANCER PATIENTS: SYSTEMATIC REVIEW

\begin{tabular}{|c|c|c|c|c|c|c|c|c|c|}
\hline $\begin{array}{l}\text { Mystakidou } \\
\text { et al. [47] }\end{array}$ & $\begin{array}{l}58 \\
\text { advance } \\
\text { d cancer } \\
\text { patients } \\
(16 \\
\text { men; } 42 \\
\text { women }) \\
\end{array}$ & $\begin{array}{l}\text { Gastrointes } \\
\text { tinal, Lung, } \\
\text { Urogenital, } \\
\text { Breast, } \\
\text { Other } \\
\text { (Stage IV) } \\
\end{array}$ & $\begin{array}{l}<3 \text { years: } \\
32 \\
\geq 3 \text { years: } \\
26\end{array}$ & $\begin{array}{l}\text { Cross- } \\
\text { sectional }\end{array}$ & PTGI & $\begin{array}{l}M=52.33 \\
S D=21.22\end{array}$ & PTSD symptoms & IES-R & $\begin{array}{l}\text { IES-R total score and its domains did } \\
\text { not predict PTG. }\end{array}$ \\
\hline $\begin{array}{l}\text { Mystakidou } \\
\text { et al. [48] }\end{array}$ & $\begin{array}{l}100 \\
\text { women }\end{array}$ & $\begin{array}{l}\text { Breast } \\
\text { (Stage IV) }\end{array}$ & $\begin{array}{l}M=6.11 \\
\text { years } \\
S D=5.0 \\
\text { years }\end{array}$ & $\begin{array}{l}\text { Cross- } \\
\text { sectional }\end{array}$ & PTGI & $\begin{array}{l}M=43.76 \\
S D=16.21\end{array}$ & $\begin{array}{l}\text { Age, marital status, } \\
\text { education, metastasis } \\
\text { time, number of } \\
\text { metastasis, } \\
\text { radiotherapy, } \\
\text { anxiety and } \\
\text { depression }\end{array}$ & HADS & $\begin{array}{l}\text { Younger age and marital status were } \\
\text { significant predictors of PTG. } \\
\text { Moreover, age is a significant predictor } \\
\text { of New Possibilities, Appreciation of } \\
\text { Life and PTGI-Total, while marital } \\
\text { status is a significant predictor of } \\
\text { PTGI-Total. }\end{array}$ \\
\hline $\begin{array}{l}\text { Park et al. } \\
\text { [49] }\end{array}$ & $\begin{array}{l}167(59 \\
\text { men; } \\
108 \\
\text { women })\end{array}$ & $\begin{array}{l}\text { Breast; } \\
\text { Prostate; } \\
\text { Colon/Rect } \\
\text { al; Lymph; } \\
\text { Cervix/Ute } \\
\text { rus } \\
\text { (Stage nr) }\end{array}$ & $\begin{array}{l}M=3.5 \\
\text { years } \\
S D=1.7 \\
\text { years }\end{array}$ & $\begin{array}{l}\text { Longitudinal } \\
\text { T1 = Baseline; } \\
\text { T2 = 1 year } \\
\text { after T1 }\end{array}$ & PBS & $\begin{array}{l}M=8.62 \\
S D=7.09\end{array}$ & $\begin{array}{l}\text { Religiousness, } \\
\text { hope/agency, } \\
\text { religious and active } \\
\text { coping }\end{array}$ & $\begin{array}{l}\text { Brief } \\
\text { MMRS }^{54} \\
\text { HS } \\
\text { Brief COPE }\end{array}$ & $\begin{array}{l}\text { Religious coping predicts perceived } \\
\text { benefits. }\end{array}$ \\
\hline $\begin{array}{l}\text { Posluszny } \\
\text { et al. [51] }\end{array}$ & $\begin{array}{l}69 \\
\text { women }\end{array}$ & $\begin{array}{l}\text { Gynecolog } \\
\text { ic } \\
\text { (endometri } \\
\text { al, ovarian, } \\
\text { vulvar, } \\
\text { benign) } \\
\text { (Stage I- } \\
\text { IV) } \\
\end{array}$ & $\begin{array}{l}M=16 \\
\text { months }\end{array}$ & $\begin{array}{l}\text { Longitudinal } \\
\text { T1 = } 1 \text { week } \\
\text { before surgery; } \\
\text { T2 = } 16 \\
\text { months after } \\
\text { T1 }\end{array}$ & $\begin{array}{l}\text { PTGI } \\
\text { (at } \\
\text { T2) }\end{array}$ & $\begin{array}{l}\text { Range: } M= \\
12.4 ; S D= \\
25.6 \text { (no } \\
\text { disease) to } M \\
=69.0 \text { to } S D \\
=25.3 \\
\text { (advanced } \\
\text { stage) }\end{array}$ & $\begin{array}{l}\text { Age, education, } \\
\text { employment, } \\
\text { income, marital } \\
\text { status, family history } \\
\text { of cancer, perceived } \\
\text { threat, PTSD } \\
\text { symptoms }\end{array}$ & PCL-C & $\begin{array}{l}\text { Higher PTSD symptoms presurgery, } \\
\text { greater disease severity and lower } \\
\text { income were significant predictors of } \\
\text { PTG. } \\
\text { The disease groups (Advanced stage; } \\
\text { Early stage; Benign) reported higher } \\
\text { levels of PTG than the no disease } \\
\text { group. }\end{array}$ \\
\hline $\begin{array}{l}\text { Rahmani et } \\
\text { al. [52] }\end{array}$ & $\begin{array}{l}450 \\
(360 \\
\text { men; } 90 \\
\text { women) } \\
\end{array}$ & $\begin{array}{l}\text { Mixed } \\
\text { (Stage nr) }\end{array}$ & $\begin{array}{l}M=29.4 \\
\text { months } \\
S D=30.4 \\
\text { months }\end{array}$ & $\begin{array}{l}\text { Cross- } \\
\text { sectional }\end{array}$ & PTGI & $\begin{array}{l}M=76.1 \\
S D=18.3\end{array}$ & $\begin{array}{l}\text { Gender, marital } \\
\text { status, education, } \\
\text { chemotherapy, } \\
\text { radiotherapy, surgery }\end{array}$ & & $\begin{array}{l}\text { PTG had a significant negative } \\
\text { association with age. Greater PTG is } \\
\text { associated with education at university } \\
\text { level and radiotherapy. }\end{array}$ \\
\hline $\begin{array}{l}\text { Rand et al. } \\
{[53]}\end{array}$ & 86 men & $\begin{array}{l}\text { Gastrointes } \\
\text { tinal; } \\
\text { Genitourin } \\
\text { ary; } \\
\text { Sarcoma; } \\
\text { Thoracic } \\
\\
\text { (Stage IV) }\end{array}$ & $\mathrm{nr}$ & $\begin{array}{l}\text { Cross- } \\
\text { sectional }\end{array}$ & PTGI & $\begin{array}{l}M=75.76 \\
S D=23.78\end{array}$ & $\begin{array}{l}\text { Age, minority, } \\
\text { education, partner } \\
\text { status, disease status, } \\
\text { prognosis (6 } \\
\text { months), deceased at } \\
6 \text { months, illness } \\
\text { appraisal, religious } \\
\text { coping, } \\
\text { psychological } \\
\text { distress, marital } \\
\text { adjustment }\end{array}$ & $\begin{array}{l}\text { Oncologist } \\
\text { prognosis } \\
\text { (Death at } 6 \\
\text { months) } \\
\text { CMS }^{55} \\
\text { Brief } \\
\text { RCOPE }^{56} \\
\text { HADS } \\
\text { Mini MAC }\end{array}$ & Religious coping predicts PTG. \\
\hline $\begin{array}{l}\text { Ruini et al. } \\
{[54]}\end{array}$ & $\begin{array}{l}120(60 \\
\text { breast } \\
\text { cancer } \\
\text { survivor } \\
\text { s }(\mathrm{BC}) ; \\
60 \\
\text { healthy } \\
\text { subjects } \\
(\mathrm{HS}))\end{array}$ & $\begin{array}{l}\text { Breast } \\
\text { (Stage nr) }\end{array}$ & $\begin{array}{l}M=6.42 \\
\text { years } \\
S D=4.06 \\
\text { years }\end{array}$ & $\begin{array}{l}\text { Cross- } \\
\text { sectional }\end{array}$ & PTGI & $\begin{array}{l}\text { BC with } \mathrm{AO} \\
(M=71.30 ; \\
S D=4.34) ; \\
\text { BC without } \\
\mathrm{AO}(M= \\
61.66 ; S D= \\
4.18) ; \mathrm{HS} \\
\text { with } \mathrm{AO}(M \\
=53.05 ; S D \\
=5.17) ; \mathrm{HS} \\
\text { without } \mathrm{AO} \\
(M=62.71 ; \\
S D=3.86)\end{array}$ & $\begin{array}{l}\text { Age, time since } \\
\text { event, marital status, } \\
\text { work status, life } \\
\text { events, distress and } \\
\text { well-being, } \\
\text { psychological well- } \\
\text { being, psychosocial } \\
\text { problems }\end{array}$ & $\begin{array}{l}\text { IRLE }^{58} \\
\text { SQ }^{59} \\
\text { PWB } \\
\text { PSI }^{60}\end{array}$ & $\begin{array}{l}\text { Women with breast cancer had higher } \\
\text { scores on PTG, than healthy women } \\
\text { with allostatic overload (AO). }\end{array}$ \\
\hline $\begin{array}{l}\text { Ruini et al. } \\
\text { [55] }\end{array}$ & & & & & & $\begin{array}{l}\mathrm{BC}(M= \\
66.74 ; S D= \\
20.98) \mathrm{HS} \\
(M=59.25 \\
S D=24.68)\end{array}$ & & & $\begin{array}{l}\text { BC survivors report higher levels of } \\
\text { PTG when compared to HS. } \\
\text { BC survivors with high levels of PTG } \\
\text { report increased levels of physical } \\
\text { well-being and decreased distress. } \\
\text { PTG levels are related with decreased } \\
\text { psychological distress and } \\
\text { somatization. }\end{array}$ \\
\hline $\begin{array}{l}\text { Salsman, et } \\
\text { al. [56] }\end{array}$ & $\begin{array}{l}55(23 \\
\text { men; } 32 \\
\text { women })\end{array}$ & $\begin{array}{l}\text { Colorectal } \\
\text { (Stage 0- } \\
\text { III) }\end{array}$ & $\begin{array}{l}M=1.07 \\
\text { years } \\
S D=.19 \\
\text { years }\end{array}$ & $\begin{array}{l}\text { Longitudinal } \\
\mathrm{T} 1=6-18 \\
\text { months post- } \\
\text { diagnosis; } \mathrm{T} 2 \\
=3 \text { months } \\
\text { after } \mathrm{T} 1\end{array}$ & PTGI & $\begin{array}{l}\mathrm{T} 1(M= \\
43.8 ; S D= \\
29.6) ; \mathrm{T} 2(M \\
=51.5 ; S D= \\
30.1)\end{array}$ & $\begin{array}{l}\text { Age, education, } \\
\text { social desirability, } \\
\text { intrusive and } \\
\text { avoidant cognition, } \\
\text { repetitive thoughts, } \\
\text { mental health, PTSD } \\
\text { symptoms }\end{array}$ & $\begin{array}{l}\text { MC-C } \\
\text { IES } \\
\text { RS }^{62} \\
\text { MHI }^{63} \\
\text { PCL-C }\end{array}$ & $\begin{array}{l}\text { PTG was not significant associated } \\
\text { with PTSD symptoms, anxiety, } \\
\text { depression, positive affectivity and } \\
\text { social desirability. } \\
\text { There was a trend for baseline } \\
\text { cognitive rehearsal predicting 3-month } \\
\text { PTG. }\end{array}$ \\
\hline $\begin{array}{l}\text { Schmidt et } \\
\text { al. [57] }\end{array}$ & $\begin{array}{l}54(14 \\
\text { men; } 40 \\
\text { women })\end{array}$ & $\begin{array}{l}\text { Breast; } \\
\text { Prostate; } \\
\text { Other } \\
\text { (Stage nr) }\end{array}$ & $\begin{array}{l}M=4.5 \\
\text { years } \\
S D=2.8 \\
\text { years }\end{array}$ & $\begin{array}{l}\text { Cross- } \\
\text { sectional }\end{array}$ & PTGI & $\mathrm{nr}$ & $\begin{array}{l}\text { Age, gender, } \\
\text { education, cancer } \\
\text { type, months since } \\
\text { diagnosis, attachment } \\
\text { styles, coping, social } \\
\text { support }\end{array}$ & $\begin{array}{l}\text { MAQ }^{64} \\
\text { Brief COPE } \\
\operatorname{MOS}^{65}\end{array}$ & $\begin{array}{l}\text { Positive reframing and religious coping } \\
\text { were predictors of PTG. }\end{array}$ \\
\hline
\end{tabular}




\begin{tabular}{|c|c|c|c|c|c|c|c|c|c|}
\hline $\begin{array}{l}\text { Schroevers } \\
\text { et al. [58] }\end{array}$ & $\begin{array}{l}108(39 \\
\text { men; } 69 \\
\text { women })\end{array}$ & $\begin{array}{l}\text { Lymphoma } \\
\text {, Breast, } \\
\text { Leukemia, } \\
\text { Colorectal, } \\
\text { Lung, } \\
\text { Prostate } \\
\text { (Stage I- } \\
\text { IV) }\end{array}$ & $\begin{array}{l}M=7.3 \\
\text { years }\end{array}$ & $\begin{array}{l}\text { Cross- } \\
\text { sectional }\end{array}$ & BFS & $\begin{array}{l}M=46.01 \\
S D=8.13\end{array}$ & $\begin{array}{l}\text { Negative } \\
\text { psychological } \\
\text { changes, positive and } \\
\text { negative affect, } \\
\text { coping, goal } \\
\text { engagement }\end{array}$ & $\begin{array}{l}\text { NPC }^{66} \\
\text { PANAS } \\
\text { CERQ }^{67} \\
\text { COPE } \\
\text { GDGDS }^{68}\end{array}$ & $\begin{array}{l}\text { Positive reappraisal and goal } \\
\text { reengagement were significant } \\
\text { predictors of BF. } \\
\text { A greater BF was significantly related } \\
\text { only to more positive affect and not to } \\
\text { negative affect. }\end{array}$ \\
\hline $\begin{array}{l}\text { Schroevers } \\
\text { and Teo } \\
{[59]}\end{array}$ & $\begin{array}{l}113(38 \\
\text { men; } 75 \\
\text { women })\end{array}$ & $\begin{array}{l}\text { Breast, } \\
\text { Nasophary } \\
\text { ngeal } \\
\text { Carcinoma, } \\
\text { Colorectal, } \\
\text { Lung } \\
\text { (Stage I- } \\
\text { IV) }\end{array}$ & $\begin{array}{l}M=45 \\
\text { months } \\
S D= \\
40.53\end{array}$ & $\begin{array}{l}\text { Cross- } \\
\text { sectional }\end{array}$ & PTGI & $\begin{array}{l}M=73.12 \\
S D=19.75\end{array}$ & $\begin{array}{l}\text { Chemotherapy, } \\
\text { coping, } \\
\text { psychological } \\
\text { distress }\end{array}$ & $\begin{array}{l}\text { Brief COPE } \\
\text { SCL-90-R }\end{array}$ & 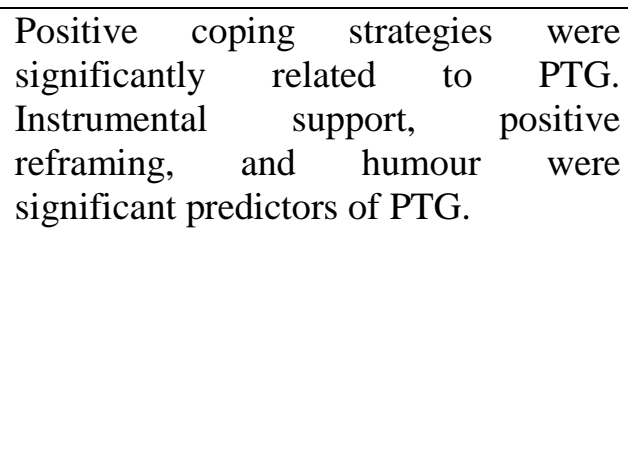 \\
\hline $\begin{array}{l}\text { Schulz and } \\
\text { Mohamed } \\
{[60]}\end{array}$ & \begin{tabular}{|l|}
$105(69$ \\
men; \\
41 \\
women $)$
\end{tabular} & $\begin{array}{l}\text { Colon; } \\
\text { Stomach; } \\
\text { Rectum; } \\
\text { Esophagus; } \\
\text { Liver and } \\
\text { gall } \\
\text { bladder; } \\
\text { Pancreas } \\
\text { (Stage nr) }\end{array}$ & $\mathrm{nr}$ & $\begin{array}{l}\text { Longitudinal } \\
\text { T1 = 1-3 days } \\
\text { before surgery } \\
\text { T2 = 5-7 days } \\
\text { after surgery } \\
\mathrm{T} 3=1 \text { months } \\
\text { after surgery } \\
\mathrm{T} 4=6 \text { months } \\
\text { after surgery } \\
\mathrm{T} 5=12 \\
\text { months after } \\
\text { surgery }\end{array}$ & $\begin{array}{l}\text { BFS } \\
\text { (at } \\
\text { T5) }\end{array}$ & $\begin{array}{l}M=3.56 \\
S D=.90\end{array}$ & $\begin{array}{l}\text { Self- efficacy, social } \\
\text { support, coping, } \\
\text { depression, quality of } \\
\text { life }\end{array}$ & $\begin{array}{l}\text { GSES }^{70} \\
\text { BSSS }^{71} \\
\text { CSSS }^{72} \\
\text { CES-D } \\
\text { EORTC- } \\
\text { QLQ30 }\end{array}$ & $\begin{array}{l}\text { Social support is the strongest predictor } \\
\text { of BF }\end{array}$ \\
\hline $\begin{array}{l}\text { Scrignaro et } \\
\text { al. [61] }\end{array}$ & $\begin{array}{l}\text { T2 } \\
n=41 \\
(90 \% \\
\text { women, } \\
10 \% \\
\text { men) }\end{array}$ & $\begin{array}{l}\text { Breast and } \\
\text { Gastroentri } \\
\text { c } \\
\text { (Stages II- } \\
\text { IV) }\end{array}$ & $\mathrm{nr}$ & $\begin{array}{l}\text { Longitudinal } \\
\text { T1 = baseline; } \\
\text { T2 = } 6 \text { months } \\
\text { after } 1 \text { 1 }\end{array}$ & PTGI & $\begin{array}{l}\mathrm{T} 1(M= \\
3.29 ; S D= \\
1.36) ; \mathrm{T} 2(M \\
=3.27 ; S D= \\
0.93)\end{array}$ & $\begin{array}{l}\text { Autonomy, support } \\
\text { environment, social } \\
\text { support, coping }\end{array}$ & $\begin{array}{l}\text { NSRS }^{73} \\
\text { ISEL }^{74} \\
\text { Brief COPE }\end{array}$ & $\begin{array}{l}\text { Autonomy-supportive caregivers (T1) } \\
\text { and a problem-focused strategy of } \\
\text { coping (T1) significantly predict } \\
\text { greater PTG at T2. } \\
\begin{array}{l}\text { At T1, PTG was significantly } \\
\text { correlated with } \\
\text { reinterpretation, positive } \\
\text { seeking instrumental support, active } \\
\text { coping, turning to religion, humour, } \\
\text { planning, perceived ability of support. }\end{array}\end{array}$ \\
\hline $\begin{array}{l}\text { Silva et al. } \\
{[62]}\end{array}$ & $\begin{array}{l}50 \\
\text { women }\end{array}$ & $\begin{array}{l}\text { Breast } \\
\text { (Stage I- } \\
\text { III) }\end{array}$ & $\begin{array}{l}M=1.36 \\
\text { months } \\
S D=.63 \\
\text { months }\end{array}$ & $\begin{array}{l}\text { Longitudinal } \\
\text { T1 = time of } \\
\text { surgery; } \mathrm{T} 2= \\
\text { during } \\
\text { adjuvant } \\
\text { treatment; } \mathrm{T} 3 \\
=6 \text { months } \\
\text { after the end of } \\
\text { treatment }\end{array}$ & $\begin{array}{l}\text { PTGI } \\
\text { (at } \\
\text { T2 } \\
\text { and } \\
\text { T3) }\end{array}$ & $\begin{array}{l}\mathrm{T} 2(M= \\
62.1 ; S D= \\
22.4) ; \mathrm{T} 3(M \\
=63.3 ; S D= \\
21.2)\end{array}$ & $\begin{array}{l}\text { Coping, } \\
\text { psychological quality } \\
\text { of life, anxiety and } \\
\text { depression }\end{array}$ & $\begin{array}{l}\text { Brief COPE } \\
\text { WHOQOL - } \\
\text { Bref } \\
\text { HADS }\end{array}$ & $\begin{array}{l}\text { The type of surgery and adjuvant } \\
\text { treatment did not account for the } \\
\text { course of PTG. } \\
\text { Domains of PTGI were significantly } \\
\text { correlated with coping, anxiety, } \\
\text { depression and psychological QoL: } \\
\text { Social support coping (T1) was } \\
\text { associated with New Possibilities and } \\
\text { Life Appreciation (T2), Strengthening } \\
\text { of Social Relationships (T2) and } \\
\text { Personal Resources and Skills (T2); } \\
\text { Cognitive coping (T1) was associated } \\
\text { with Personal Resources and Skills } \\
\text { (T2); Personal Resources and Skills } \\
\text { (T2) was associated with anxiety, } \\
\text { depression and psychological QoL } \\
\text { (T3); Strengthening of Social } \\
\text { Relationships (T2) was associated with } \\
\text { psychological QoL (T3); }\end{array}$ \\
\hline $\begin{array}{l}\text { Smith et al. } \\
{[63]}\end{array}$ & \begin{tabular}{|l|}
173 \\
$(132$ \\
Non \\
Hispani \\
c White \\
(NHW); \\
51 \\
Hispani \\
c \\
(HISP)) \\
\end{tabular} & $\begin{array}{l}\text { Cervical } \\
\text { (Stage nr) }\end{array}$ & $\begin{array}{l}M=10.30 \\
\text { years } \\
S D=5.01 \\
\text { years }\end{array}$ & $\begin{array}{l}\text { Cross- } \\
\text { sectional }\end{array}$ & PTGI & $\begin{array}{l}M=2.30 \\
S D=1.40\end{array}$ & $\begin{array}{l}\text { Age, education, } \\
\text { income, cancer stage, } \\
\text { years since } \\
\text { diagnosis, optimism, } \\
\text { spirituality }\end{array}$ & $\begin{array}{l}\text { LOT-R } \\
\text { DRI }\end{array}$ & $\begin{array}{l}\text { Greater spirituality and more advanced } \\
\text { cancer stage predicted more PTG, but } \\
\text { optimism did not predict PTG. } \\
\text { HISP women reported higher levels of } \\
\text { PTG than NHW women. }\end{array}$ \\
\hline $\begin{array}{l}\text { Smith et al. } \\
{[64]}\end{array}$ & $\begin{array}{l}886 \\
(435 \\
\text { men; } \\
451 \\
\text { women })\end{array}$ & $\begin{array}{l}\text { Non- } \\
\text { Hodgkin } \\
\text { lymphoma } \\
\text { (Stage I- } \\
\text { IV) }\end{array}$ & $\begin{array}{l}M=10.2 \\
\text { years } S D \\
=7.10 \\
\text { years }\end{array}$ & $\begin{array}{l}\text { Cross- } \\
\text { sectional }\end{array}$ & \begin{tabular}{|l} 
PTGI \\
\end{tabular} & $\begin{array}{l}M=60.5 \\
S D=24.7\end{array}$ & $\begin{array}{l}\text { Gender, race, } \\
\text { income, education, } \\
\text { age, years since } \\
\text { diagnosis, stage, type } \\
\text { of lymphoma, not } \\
\text { currently receiving } \\
\text { treatment, } \\
\text { chemotherapy, } \\
\text { transplant, } \\
\text { comorbidity, } \\
\text { psychological status, }\end{array}$ & $\begin{array}{l}\text { MOS-SSS }^{75} \\
\text { ALTTIQ }^{76} \\
\text { PCL-C } \\
\text { CCI }\end{array}$ & $\begin{array}{l}\text { PTG was positively associated with } \\
\text { social support and perceived life- } \\
\text { threatening of cancer. } \\
\text { Greater PTG was significant associated } \\
\text { to female gender, non-Caucasian race, } \\
\text { having less than a college degree, } \\
\text { younger age and having stage of cancer } \\
>1 \text {. }\end{array}$ \\
\hline
\end{tabular}


PTG IN ADULT CANCER PATIENTS: SYSTEMATIC REVIEW

\begin{tabular}{|c|c|c|c|c|c|c|c|c|c|}
\hline & & & & & & & $\begin{array}{l}\text { perceived life- } \\
\text { threatening of } \\
\text { cancer, PTSD } \\
\text { symptoms }\end{array}$ & & \\
\hline $\begin{array}{l}\text { Soo and } \\
\text { Sherman } \\
{[65]}\end{array}$ & $\begin{array}{l}185 \\
\text { women }\end{array}$ & $\begin{array}{l}\text { Breast } \\
\text { (Stage I- } \\
\text { IV) }\end{array}$ & $\begin{array}{l}1-6 \\
\text { months: } \\
N=6 \\
7 \text { months- } \\
1 \text { year: } \\
N=11 \\
1-4 \text { years: } \\
N=68 \\
5-10 \\
\text { years: } \\
N=71 \\
10 \text { years } \\
\text { plus: } \\
N=29 \\
\end{array}$ & $\begin{array}{l}\text { Cross- } \\
\text { sectional }\end{array}$ & PTGI & $\begin{array}{l}M=48.55 \\
S D=20.58\end{array}$ & $\begin{array}{l}\text { Education, time since } \\
\text { diagnosis, treatment, } \\
\text { comorbid } \\
\text { psychological, } \\
\text { rumination, } \\
\text { depressive, anxious } \\
\text { and stress } \\
\text { symptomatology, } \\
\text { multiple dimensions } \\
\text { of support }\end{array}$ & $\begin{array}{l}\text { MDRIS }^{77} \\
\text { MOS-SS } \\
\text { DASS }^{78}\end{array}$ & $\begin{array}{l}\text { Brooding was significant predictor of } \\
\text { New Possibilities; Intrusion and } \\
\text { Instrumentality were significant } \\
\text { predictors of New Possibilities and } \\
\text { Relating to Others; and Instrumentality } \\
\text { for Personal strength and Appreciation } \\
\text { of life. } \\
\text { Brooding and instrumentality predicted } \\
\text { spiritual change. }\end{array}$ \\
\hline $\begin{array}{l}\text { Strack et al. } \\
{[67]}\end{array}$ & $\begin{array}{l}128(51 \\
\text { men; } 77 \\
\text { women })\end{array}$ & $\begin{array}{l}\text { Mixed } \\
\text { (Stage nr) }\end{array}$ & NR & $\begin{array}{l}\text { Cross- } \\
\text { sectional }\end{array}$ & PTGI & $\begin{array}{l}\mathrm{M}=5.09 \\
\mathrm{SD}=.88\end{array}$ & $\begin{array}{l}\text { Openness to } \\
\text { experience, cognitive } \\
\text { reappraisal, } \\
\text { gratitude, priorities } \\
\text { in life }\end{array}$ & $\begin{array}{l}\mathrm{BFI}^{79} \\
\mathrm{ERQ}^{80} \\
\mathrm{GQ}^{81}\end{array}$ & $\begin{array}{l}\text { Openness to experience, gratitude and } \\
\text { cognitive reappraisal of emotion were } \\
\text { positively associated with PTG. }\end{array}$ \\
\hline $\begin{array}{l}\text { Svetina and } \\
\text { Nastran } \\
{[68]}\end{array}$ & $\begin{array}{l}190 \\
\text { women }\end{array}$ & $\begin{array}{l}\text { Breast } \\
\text { (Stage nr) }\end{array}$ & NR & $\begin{array}{l}\text { Cross- } \\
\text { sectional }\end{array}$ & PTGI & $\begin{array}{l}\text { ( PTG } \\
\text { believers } M \\
=72.58 \text { ) } \\
\text { (PTG non- } \\
\text { believers } \\
M=70.15 \text { ) }\end{array}$ & $\begin{array}{l}\text { Education level, age, } \\
\text { marital status, } \\
\text { number of children, } \\
\text { currently living with } \\
\text { their family } \\
\text { members, cancer in } \\
\text { acute or remission } \\
\text { state, length of } \\
\text { treatment, religious } \\
\text { affiliation, } \\
\text { volunteering, family } \\
\text { relationships, coping }\end{array}$ & $\begin{array}{l}\text { FACES IV } \\
\text { CRI }^{83}\end{array}$ & $\begin{array}{l}\text { Approach related coping strategies and } \\
\text { family related factors predict PTG. }\end{array}$ \\
\hline $\begin{array}{l}\text { Tang et al. } \\
{[69]}\end{array}$ & $\begin{array}{l}313 \\
(179 \\
\text { men; } \\
134 \\
\text { women })\end{array}$ & $\begin{array}{l}\text { Stomach; } \\
\text { Liver; } \\
\text { Pancreas; } \\
\text { Head and } \\
\text { neck; } \\
\text { Lung; } \\
\text { Esophagus; } \\
\text { Colon- } \\
\text { rectum; } \\
\text { Other } \\
\text { (Stage IV) }\end{array}$ & $\begin{array}{l}M=19.28 \\
\text { months } \\
S D= \\
33.94 \\
\text { months }\end{array}$ & $\begin{array}{l}\text { Longitudinal } \\
\text { T1 - diagnosis } \\
\text { of terminal } \\
\text { status; } \\
\text { Next } \\
\text { assessments } \\
\text { were made } \\
\text { approximately } \\
\text { every } 2 \text { weeks } \\
\text { from } \\
\text { enrollment } \\
\text { until they } \\
\text { declined to } \\
\text { participate or } \\
\text { died. }\end{array}$ & PTGI & $\begin{array}{l}\text { Range from } \\
M=26.13 \\
S D=21.54 \\
\text { to } M=40.33 \\
S D=27.71\end{array}$ & $\begin{array}{l}\text { Time proximity to } \\
\text { patient death, gender, } \\
\text { age, educational } \\
\text { level, marital status, } \\
\text { with chronic disease, } \\
\text { metastasis, post- } \\
\text { diagnosis survival at } \\
\text { enrollment, time } \\
\text { since recognition of } \\
\text { terminally ill status, } \\
\text { distress, functional } \\
\text { dependency, social } \\
\text { support, coping } \\
\text { strategies }\end{array}$ & $\begin{array}{l}\text { SDS }^{84} \\
\text { ESDS }^{85} \\
\text { MOS-SSS }\end{array}$ & $\begin{array}{l}\text { Patients reported higher PTG if they } \\
\text { were female, have higher education, } \\
\text { have a metastatic disease, and reported } \\
\text { higher social support. PTG was lower } \\
\text { in patients who had recently } \\
\text { recognized their terminally ill status, } \\
\text { have distress or have high functional } \\
\text { dependence, were unaware or had low } \\
\text { acceptance of their diagnosis. }\end{array}$ \\
\hline $\begin{array}{l}\text { Tanriverd et } \\
\text { al. [70] }\end{array}$ & $\begin{array}{l}105(46 \\
\text { men; } 74 \\
\text { women })\end{array}$ & $\begin{array}{l}\text { Mixed } \\
\text { (Stage nr) }\end{array}$ & $\mathrm{nr}$ & $\begin{array}{l}\text { Cross- } \\
\text { sectional }\end{array}$ & PTGI & $\begin{array}{l}M=57.14 \\
S D=16.52\end{array}$ & $\begin{array}{l}\text { Perceived social } \\
\text { support }\end{array}$ & MSPSS & $\begin{array}{l}\text { Total perceived social support, support } \\
\text { from family, and friends were } \\
\text { significantly positive correlated with } \\
\text { PTG. }\end{array}$ \\
\hline $\begin{array}{l}\text { Tanyi et al. } \\
\text { [71] }\end{array}$ & $\begin{array}{l}91(14 \\
\text { men; } 77 \\
\text { women })\end{array}$ & $\begin{array}{l}\text { Breast; } \\
\text { Prostate } \\
\text { (Stage I- } \\
\text { IV) }\end{array}$ & $\begin{array}{l}M=111.2 \\
\text { days } \\
S D=77.5 \\
\text { days }\end{array}$ & $\begin{array}{l}\text { Longitudinal } \\
\text { T1 = one or } \\
\text { two weeks } \\
\text { before the start } \\
\text { of } \\
\text { Radiotherapy; } \\
\text { T2 = } \\
\text { fifth/sixth } \\
\text { week of } \\
\text { treatment } \\
\text { T3 = four to } \\
\text { six weeks } \\
\text { post-treatment }\end{array}$ & PTGI & $\begin{array}{l}\text { T1 }(M=63 \\
S D=23.3) \\
\text { T2 }(M= \\
67.5 ; S D= \\
22.3) ; \mathrm{T} 3(M \\
=64.9 ; S D= \\
23.9)\end{array}$ & $\begin{array}{l}\text { Health-related } \\
\text { quality of life }\end{array}$ & FACT-G & $\begin{array}{l}\text { A significant negative correlation was } \\
\text { found between Physical Well-being } \\
\text { and PTG, whereas a significant } \\
\text { positive correlation was revealed } \\
\text { between Social/Family Well-being and } \\
\text { PTG. }\end{array}$ \\
\hline $\begin{array}{l}\text { Tanyi et al. } \\
\text { [72] }\end{array}$ & $\begin{array}{l}152(29 \\
\text { men; } \\
113 \\
\text { women })\end{array}$ & $\begin{array}{l}\text { Breast; } \\
\text { Prostate } \\
\text { (Stage I- } \\
\text { IV) }\end{array}$ & $\begin{array}{l}M=3.5 \\
\text { months } \\
S D=2.2 \\
\text { months }\end{array}$ & $\begin{array}{l}\text { Cross- } \\
\text { sectional }\end{array}$ & PTGI & $\begin{array}{l}M=65.53 \\
S D=22.07\end{array}$ & $\begin{array}{l}\text { Adult attachment } \\
\text { style, health-related } \\
\text { quality of life, } \\
\text { subjective severity } \\
\text { and perceived } \\
\text { aftermath of cancer }\end{array}$ & $\begin{array}{l}\text { ECRS }^{86} \\
\text { FACT-G } \\
2 \text { questions }\end{array}$ & $\begin{array}{l}\text { Younger age, subjective severity of } \\
\text { cancer and social/family well-being } \\
\text { were significant predictors of PTG. } \\
\text { Dismissive attachment style predicted } \\
\text { fewer score on the Personal Strength } \\
\text { and Relating to Others subscales. }\end{array}$ \\
\hline $\begin{array}{l}\text { Thombre et } \\
\text { al. [73] }\end{array}$ & $\begin{array}{l}59(29 \\
\text { men; } 30 \\
\text { women })\end{array}$ & $\begin{array}{l}\text { Breast; } \\
\text { Lung; } \\
\text { Head/Neck } \\
\text { (Stage I- } \\
\text { III) }\end{array}$ & $\begin{array}{l}M=11.3 \\
\text { months } \\
S D=30 \\
\text { months }\end{array}$ & $\begin{array}{l}\text { Cross- } \\
\text { sectional }\end{array}$ & PTGI & $\begin{array}{l}M=34.80 \\
S D=4.84\end{array}$ & $\begin{array}{l}\text { Illness appraisals, } \\
\text { meaning-based } \\
\text { coping, core beliefs, } \\
\text { fear of recurrence }\end{array}$ & $\begin{array}{l}\text { two } \\
\text { questions } \\
\text { (Vinokur et } \\
\text { al., 1990) } \\
\text { two } \\
\text { questions } \\
\end{array}$ & $\begin{array}{l}\text { Revaluation of worldviews (challenge } \\
\text { of core beliefs) was the only significant } \\
\text { predictor of PTG. } \\
\text { PTG was positively related to disease } \\
\text { recurrence. } \\
\text {. }\end{array}$ \\
\hline
\end{tabular}




\begin{tabular}{|c|c|c|c|c|c|c|c|c|c|}
\hline & & & & & & & & $\begin{array}{l}\text { (Davis et al., } \\
\text { 1998) } \\
\text { CBI }\end{array}$ & \\
\hline $\begin{array}{l}\text { Thornton et } \\
\text { al. [74] }\end{array}$ & $\begin{array}{l}118(50 \\
\text { men; } 68 \\
\text { women })\end{array}$ & $\begin{array}{l}\text { Lung } \\
\text { (Stage I- } \\
\text { IV) }\end{array}$ & $\begin{array}{l}M=16 \\
\text { weeks } \\
S D=9.32 \\
\text { weeks }\end{array}$ & $\begin{array}{l}\text { Longitudinal } \\
\text { T1 = baseline } \\
\text { T2 }=3 \text { months } \\
\text { after } \mathrm{T} 1\end{array}$ & PTGI & $\begin{array}{l}\mathrm{T} 1(M= \\
55.30 ; S D= \\
28.22) ; \mathrm{T} 2 \\
(M=52.95 \\
S D=26.22)\end{array}$ & $\begin{array}{l}\text { Gender, cancer type, } \\
\text { coping, cancer- } \\
\text { related stress }\end{array}$ & $\begin{array}{l}\text { IES-R } \\
\text { COPE } \\
\text { EACP }^{87}\end{array}$ & $\begin{array}{l}\text { Greater growth was associated with } \\
\text { having small cell lung cancer, higher } \\
\text { cancer-related intrusions, lower } \\
\text { perceived cancer-related stress, and } \\
\text { greater approach-oriented coping. } \\
\text { Positive reframing coping predicts } \\
\text { growth (T1) and emotional approach } \\
\text { coping predicts growth (T2). }\end{array}$ \\
\hline $\begin{array}{l}\text { Thuné- } \\
\text { Boyle et al. } \\
{[75]}\end{array}$ & $\begin{array}{l}202 \\
\text { women }\end{array}$ & $\begin{array}{l}\text { Breast } \\
\text { (Stage nr) }\end{array}$ & $\mathrm{nr}$ & Prospective & BFS & $\mathrm{nr}$ & $\begin{array}{l}\text { Religious/spiritual } \\
\text { beliefs, } \\
\text { Private } \\
\text { religious/spiritual } \\
\text { practice, } \\
\text { religiousness/spiritua } \\
\text { lity, public } \\
\text { religious/spiritual } \\
\text { practice, religious } \\
\text { coping, private } \\
\text { spiritual } \\
\text { involvement, } \\
\text { perceived spiritual } \\
\text { support, non- } \\
\text { religious coping, } \\
\text { optimism, social- } \\
\text { support }\end{array}$ & $\begin{array}{l}\text { MMR/S } \\
\text { RCOPE } \\
\text { SIS }^{89} \\
\text { SSS }^{90} \\
\text { Brief COPE } \\
\text { LOT } \\
\text { MSPSS }\end{array}$ & $\begin{array}{l}\text { Strength of faith at surgery and seeking } \\
\text { emotional support predicts } \mathrm{BF} \text { at three } \\
\text { months. Relationship between } \mathrm{BF} \text { and } \\
\text { religious coping to achieve a life } \\
\text { transformation was partially mediated } \\
\text { by strength of faith. }\end{array}$ \\
\hline $\begin{array}{l}\text { Tomich and } \\
\text { Helgeson } \\
{[76]}\end{array}$ & $\begin{array}{l}62(26 \\
\text { men; } 36 \\
\text { women })\end{array}$ & $\begin{array}{l}\text { Colorectal; } \\
\text { Lung } \\
\text { (Stage II- } \\
\text { IV) }\end{array}$ & $\mathrm{nr}$ & $\begin{array}{l}\text { Longitudinal } \\
\text { T1 = on } \\
\text { average } 3- \\
\text { months } \\
\text { postdiagnosis; } \\
\text { T2 = } 3 \text { months } \\
\text { after T1 }\end{array}$ & PTGI & $\begin{array}{l}\mathrm{T} 1(M= \\
3.37 ; S D= \\
.82) ; \mathrm{T} 2(M \\
=3.34 ; S D= \\
.75)\end{array}$ & $\begin{array}{l}\text { Health-related } \\
\text { quality of life, } \\
\text { depressive } \\
\text { symptoms, } \\
\text { stressfulness of } \\
\text { cancer, intrusive } \\
\text { thoughts, coping }\end{array}$ & $\begin{array}{l}\text { SF-36 } \\
\text { CES-D } \\
\text { IES } \\
\text { COPE }\end{array}$ & $\begin{array}{l}\text { More PTG was related to worse mental } \\
\text { health at T1 but was not related to } \\
\text { physical health (T1) or depressive } \\
\text { symptoms (T1) } \\
\text { More PTG at T1 predicted better } \\
\text { physical health at T2. }\end{array}$ \\
\hline $\begin{array}{l}\text { Wang et al. } \\
\text { [77] }\end{array}$ & $\begin{array}{l}124 \\
\text { women }\end{array}$ & $\begin{array}{l}\text { Breast } \\
\text { (Stage 0- } \\
\text { IV) }\end{array}$ & $\mathrm{nr}$ & $\begin{array}{l}\text { Longitudinal } \\
\text { T1 = 1-2 days } \\
\text { after they } \\
\text { surgery; T2 = } \\
\text { 3-month; T3 = } \\
6 \text {-month; T4 = } \\
12 \text { months } \\
\text { after T1 }\end{array}$ & PTGI & $\begin{array}{l}\text { TI }(M= \\
59.27 ; S D= \\
24.67) ; \mathrm{T} 2 \\
(\mathrm{M}=55.71 ; \\
S D=26.71) ; \\
\text { T3 }=(M= \\
54.01 ; S D= \\
27.42) ; \mathrm{T} 4 \\
(M=52.47 ; \\
S D=28.24)\end{array}$ & $\begin{array}{l}\text { Anxiety and } \\
\text { depression, positive } \\
\text { and negative affect, } \\
\text { health-related quality } \\
\text { of life }\end{array}$ & $\begin{array}{l}\text { HADS } \\
\text { ABS } \\
\text { SF-36 }\end{array}$ & $\begin{array}{l}\text { The relationship between PTG and } \\
\text { positive affect was consistently } \\
\text { positive, but the relationship between } \\
\text { PTG and depression was consistently } \\
\text { negative over the time. }\end{array}$ \\
\hline $\begin{array}{l}\text { Wang et al. } \\
{[78]}\end{array}$ & $\begin{array}{l}1227 \\
\text { women }\end{array}$ & $\begin{array}{l}\text { Breast } \\
\text { (Stage 0- } \\
\text { IV) }\end{array}$ & $\begin{array}{l}\text { Median }= \\
3.5 \text { years } \\
\text { Range }= \\
4 \text { months } \\
\text { to } 26 \\
\text { years }\end{array}$ & $\begin{array}{l}\text { Cross- } \\
\text { sectional }\end{array}$ & PTGI & $\begin{array}{l}M=70.18 \\
S D=15.85\end{array}$ & $\begin{array}{l}\text { Age, time from } \\
\text { diagnosis, number of } \\
\text { children, occupation, } \\
\text { education level, } \\
\text { household income, } \\
\text { marital status, } \\
\text { exercise, work status, } \\
\text { religion, TNM stage, } \\
\text { breast cancer } \\
\text { volunteer, breast } \\
\text { cancer family } \\
\text { history, surgery, } \\
\text { comorbidity, current } \\
\text { treatment }\end{array}$ & & $\begin{array}{l}\text { PTG was positively and significantly } \\
\text { associated with household income, } \\
\text { education and exercise. PTG was } \\
\text { negatively associated with concomitant } \\
\text { chronic disease. }\end{array}$ \\
\hline $\begin{array}{l}\text { Wang et al. } \\
\text { [79] }\end{array}$ & $\begin{array}{l}404 \\
\text { women }\end{array}$ & $\begin{array}{l}\text { Breast } \\
\text { (Stage 0- } \\
\text { III) }\end{array}$ & $\begin{array}{l}\text { Range }=1 \\
\text { week to } 6 \\
\text { weeks } \\
\text { after } \\
\text { diagnosis }\end{array}$ & $\begin{array}{l}\text { Longitudinal } \\
\text { T1 = during } \\
\text { the first week } \\
\text { after the } \\
\text { confirmation } \\
\text { of the } \\
\text { diagnosis; } \mathrm{T} 2 \\
=6 \text { weeks } \\
\text { after diagnosis }\end{array}$ & BFS & $\begin{array}{l}\mathrm{T} 1(M= \\
44.95 ; S D= \\
7.60) \mathrm{T} 2(M \\
=40.84 ; S D \\
=6.03)\end{array}$ & $\begin{array}{l}\text { Age, education, } \\
\text { employment, stage of } \\
\text { disease, optimism } \\
\text { and pessimism, } \\
\text { perceived social } \\
\text { support, cognitive } \\
\text { emotion regulation }\end{array}$ & $\begin{array}{l}\text { OPS }^{91} \\
\text { MSPSS } \\
\text { CERQ }\end{array}$ & $\begin{array}{l}\text { BF (T2) was positively predicted by } \\
\text { age, education level, social support } \\
\text { from family, acceptance, positive } \\
\text { reappraisal, BF (T1). Vocational status, } \\
\text { adaptive and maladaptive coping } \\
\text { emotion regulation strategies a } \\
\text { negative significant predictor of BF } \\
\text { (T2). }\end{array}$ \\
\hline $\begin{array}{l}\text { Wilson et } \\
\text { al. [80] }\end{array}$ & $\begin{array}{l}514 \\
\text { men }\end{array}$ & $\begin{array}{l}\text { Prostate } \\
\text { (Stage nr) }\end{array}$ & $\begin{array}{l}M=7.50 \\
\text { years } \\
S D=4.66 \\
\text { years }\end{array}$ & $\begin{array}{l}\text { Cross- } \\
\text { sectional }\end{array}$ & PTGI & $\begin{array}{l}M=50.20 \\
S D=22.99\end{array}$ & $\begin{array}{l}\text { Resilience, challenge } \\
\text { appraisal, cancer } \\
\text { related distress, core } \\
\text { beliefs, rumination, } \\
\text { peer support factors, } \\
\text { social constraints }\end{array}$ & $\begin{array}{l}\text { CDRS }^{92} \\
\text { SAM }^{93} \\
\text { IES } \\
\text { CBI } \\
\text { ERRI }^{94} \\
\text { MEIM }^{95} \\
\text { SCS }\end{array}$ & $\begin{array}{l}\text { The variables with a direct effect on } \\
\text { PTG were: challenge appraisal; } \\
\text { examining core beliefs; intrusive } \\
\text { rumination and peer support factors. } \\
\text { Other variables (i.e. resilience, } \\
\text { challenge appraisal, distress and } \\
\text { examining core beliefs) have shown } \\
\text { indirect effects on PTG. }\end{array}$ \\
\hline
\end{tabular}


PTG IN ADULT CANCER PATIENTS: SYSTEMATIC REVIEW

\begin{tabular}{|c|c|c|c|c|c|c|c|c|c|}
\hline $\begin{array}{l}\text { Yu et al. } \\
{[81]}\end{array}$ & \begin{tabular}{|l}
$208(80$ \\
men; \\
128 \\
women)
\end{tabular} & $\begin{array}{l}\text { Cervical; } \\
\text { Ovarian; } \\
\text { Esophagus; } \\
\text { Lung; } \\
\text { Breast; } \\
\text { Nasophary } \\
\text { ngealcarcin } \\
\text { oma; } \\
\text { Bladder; } \\
\text { Hepatocell } \\
\text { ular } \\
\text { (Stage nr) }\end{array}$ & $\mathrm{nr}$ & $\begin{array}{l}\text { Cross- } \\
\text { sectional }\end{array}$ & PTGI & $\begin{array}{l}M=49.54 \\
S D=21.15\end{array}$ & $\begin{array}{l}\text { Age, gender, } \\
\text { education, emotion } \\
\text { regulation, positive } \\
\text { and negative affect, } \\
\text { self-efficacy }\end{array}$ & $\begin{array}{l}\text { ERS }^{96} \\
\text { PANAS } \\
\text { GSES }\end{array}$ & $\begin{array}{l}\text { PTG was positively associated with } \\
\text { positive affect, effective emotion } \\
\text { regulation (revealing and suppression), } \\
\text { and general self-efficacy. } \\
\text { No significant correlation could be } \\
\text { found between negative affect and } \\
\text { PTG. }\end{array}$ \\
\hline
\end{tabular}

Table 4.

Characteristics of studies with intervention

\begin{tabular}{|c|c|c|c|c|c|c|c|c|c|}
\hline $\begin{array}{c}\text { Study } \\
\text { (Authors, } \\
\text { date) }\end{array}$ & Sample & Cancer & $\begin{array}{c}\text { Time since } \\
\text { cancer }\end{array}$ & Design & $\begin{array}{c}\text { Interventio } \\
\mathbf{n}\end{array}$ & $\begin{array}{l}\text { Measure of growth } \\
\text { and scores }\end{array}$ & \multicolumn{2}{|c|}{ Other measures } & \multirow[t]{2}{*}{ Outcomes } \\
\hline $\begin{array}{l}\text { Antoni et } \\
\text { al. [3] }\end{array}$ & $\begin{array}{l}199 \\
\text { women } \\
\text { (74 EG; } \\
83 \mathrm{CG})\end{array}$ & $\begin{array}{l}\text { Breast } \\
\text { (Stage 0-III) }\end{array}$ & $\mathrm{nr}$ & $\begin{array}{l}\text { RCT } \\
\text { T1 = Baseline } \\
\text { (4-8 weeks } \\
\text { postsurgery); T2 } \\
=6 \text { months after } \\
\text { T1 ( } 3 \text { months } \\
\text { after end of } \\
\text { intervention); } \\
\text { T3 = } 12 \text { months } \\
\text { after T1 }\end{array}$ & $\begin{array}{l}\text { Cognitive- } \\
\text { Behavioral } \\
\text { Stress } \\
\text { Managemen } \\
\mathrm{t}(\mathrm{CBSM})\end{array}$ & $\begin{array}{l}\text { BFS } \\
\text { EG } \\
\text { T1 }(M=3.16 ; S D= \\
.20) ; \mathrm{T} 2(M=3.51 ; S D \\
=.10): \mathrm{T} 3(M=3.59 ; \\
S D=.10 \\
\text { CG } \\
\text { T1 }(M=3.32 ; S D= \\
.09) ; \mathrm{T} 2(M=3.40 ; S D \\
=.08): \mathrm{T} 3(M=3.42 ; \\
S D=.10)\end{array}$ & $\begin{array}{l}\text { Illness-related } \\
\text { interpersonal } \\
\text { disruption, } \\
\text { positive states of } \\
\text { mind, positive } \\
\text { emotional } \\
\text { experience, } \\
\text { perceived stress } \\
\text { management } \\
\text { skills, }\end{array}$ & $\begin{array}{l}\text { SIP } \\
\text { PSOM }^{97} \\
\text { MOCS }^{98} \\
\text { ABS }\end{array}$ & \\
\hline $\begin{array}{l}\text { Cameron } \\
\text { et al. [11] }\end{array}$ & $\begin{array}{l}154(54 \\
\text { EG; } 56 \\
\text { DG; } 44 \\
\text { CG) }\end{array}$ & $\begin{array}{l}\text { Breast } \\
\text { (Stage nr) }\end{array}$ & $\mathrm{nr}$ & $\begin{array}{l}\text { Quasi- } \\
\text { Experimental } \\
\text { T1 = Baseline } \\
\text { (following } \\
\text { diagnosis); T2 = } \\
4 \text { months (post- } \\
\text { intervention); } \\
\text { T3 = } 6 \text { month } \\
\text { (follow-up); T4 } \\
=12 \text { months } \\
\text { (follow-up) }\end{array}$ & $\begin{array}{l}\text { Psychosocia } \\
\text { 1 Support } \\
\text { Program }\end{array}$ & $\begin{array}{l}\text { BFS } \\
\text { EG } \\
\text { T3 }(M=61.63 ; S D= \\
12.39) ; \mathrm{T} 4(M=65.63 ; \\
S D=12.24) \\
\text { CG } \\
\text { T3 }(M=60.99 ; S D= \\
14.45) ; \mathrm{T} 4(M=59.96 ; \\
S D=18.15) \\
\text { DG } \\
\text { T3 }(M=53.21 ; S D= \\
16.85) ; \mathrm{T} 4(M=53.31 ; \\
S D=18.59)\end{array}$ & $\begin{array}{l}\text { Use of } \\
\text { relaxation- } \\
\text { related } \\
\text { techniques, } \\
\text { emotional } \\
\text { suppression, } \\
\text { perceived } \\
\text { control, } \\
\text { perceived risk of } \\
\text { recurrence, } \\
\text { emotional well- } \\
\text { being, cancer } \\
\text { worry, state } \\
\text { anxiety, coping } \\
\text { efficacy }\end{array}$ & $\begin{array}{l}\text { CECS }^{99} \\
\text { IPQ }^{100} \\
\text { FACT- } \\
\text { G } \\
\text { STAI- } \\
\text { SF } \\
\text { CEM }^{101}\end{array}$ & $\begin{array}{l}\text { EG reported higher } \mathrm{BF} \text {, at } \\
\mathrm{T} 3 \text { and } \mathrm{T} 4 \text {, when comparing } \\
\text { with } \mathrm{DG} \text {. } \\
\text { CG reported greater } \mathrm{BF} \\
\text { relative to DG at T3, } \\
\text { although these two groups } \\
\text { did not differ significantly at } \\
\text { T4. }\end{array}$ \\
\hline $\begin{array}{l}\text { Garlick et } \\
\text { al. [24] }\end{array}$ & $\begin{array}{l}24 \\
\text { women }\end{array}$ & $\begin{array}{l}\text { Breast } \\
\text { (Stage 0-III) }\end{array}$ & $\begin{array}{l}M=20.7 \\
\text { months } \\
S D=27.03 \\
\text { months }\end{array}$ & $\begin{array}{l}\text { Quasi- } \\
\text { experimental } \\
\mathrm{T} 1=\text { baseline } \\
\text { (first day of } \\
\text { intervention); } \\
\mathrm{T} 2=1 \text { week } \\
\text { post- } \\
\text { intervention; T3 } \\
\text { = follow-up (4 } \\
\text { weeks after } \\
\text { treatment) }\end{array}$ & $\begin{array}{l}\text { Psycho- } \\
\text { spiritual } \\
\text { integrative } \\
\text { therapy }\end{array}$ & $\begin{array}{l}\text { PTGI } \\
\text { T1 } \\
\text { Range sub-scales } \\
M=4.5 ; S D=3.3 \text {. to } \\
M=20.8 ; \mathrm{SD}=9.2 . \\
\text { T2 } \\
\text { Range sub-scales } \\
M=5.3 ; S D=3.2 \text {. to } \\
\mathrm{M}=23.3 ; S D=8.8 . \\
\text { T3 } \\
\text { Range sub-scales } \\
M=5.0 ; S D=3.0 . \text { to } \\
M=21.5 ; S D=6.5 .\end{array}$ & $\begin{array}{l}\text { Quality of life, } \\
\text { affective and } \\
\text { physical states, } \\
\text { spiritual well- } \\
\text { being }\end{array}$ & $\begin{array}{l}\text { FACT- } \\
\text { B } \\
\text { POMS } \\
\text { FACIT- } \\
\text { Sp-Ex }\end{array}$ & $\begin{array}{l}\text { A significant main effect for } \\
\text { time was found between the } \\
\mathrm{T} 1 \text { and } \mathrm{T} 2 \text {, on New } \\
\text { Possibilities. A significant } \\
\text { main effect for time was } \\
\text { found between } \mathrm{T} 1 \text {, } \mathrm{T} 2 \text { and } \\
\mathrm{T} 3 \text {, on Personal Strength. } \\
\text { No significant differences } \\
\text { were found on Relating to } \\
\text { Others, Spiritual Change or } \\
\text { Appreciation of Life } \\
\text { Subscale. }\end{array}$ \\
\hline $\begin{array}{l}\text { Hawkes et } \\
\text { al. [26] }\end{array}$ & $\begin{array}{l}410 \\
(221 \\
\text { men; } \\
189 \\
\text { women })\end{array}$ & $\begin{array}{l}\text { Colorectal } \\
\text { (Stage nr) }\end{array}$ & $\mathrm{nr}$ & $\begin{array}{l}\text { Longitudinal } \\
\text { T1 = baseline; } \\
\text { T2 = } 6 \text { months; } \\
\text { T3 = } 12 \text { months }\end{array}$ & $\begin{array}{l}\text { Multiple } \\
\text { Health } \\
\text { Behavior } \\
\text { Change } \\
\text { Intervention }\end{array}$ & $\begin{array}{l}\text { PTGI } \\
\text { Health coaching } \\
\text { T1 }(M=53.1 ; S D= \\
23.1) \\
\text { Usual care } \\
\text { T1 }(M=56.2 ; S D= \\
23.8)\end{array}$ & $\begin{array}{l}\text { Spirituality, } \\
\text { acceptance, } \\
\text { Mindfulness, } \\
\text { distress, cancer- } \\
\text { specific quality } \\
\text { of life }\end{array}$ & $\begin{array}{l}\text { FACIT- } \\
\text { Sp } \\
\text { AAQ }^{102} \\
\text { MAAS }^{10} \\
\text { BSI- } \\
18^{104} \\
\text { FACT- } \\
\text { C }^{105}\end{array}$ & $\begin{array}{l}\text { Significant intervention } \\
\text { effects were observed for } \\
\text { PTG at T2 and T3, in the } \\
\text { following subscales: New } \\
\text { Possibilities and Relating to } \\
\text { Others. } \\
\text { Significant effects of } \\
\text { intervention were found at } \\
\text { T2 on Appreciation of Life } \\
\text { and Personal Strength. }\end{array}$ \\
\hline $\begin{array}{l}\text { Kállay } \\
\text { and Baban } \\
{[30]}\end{array}$ & 45 & $\begin{array}{l}\text { Breast, } \\
\text { Cervical, } \\
\text { Pulmonary } \\
\text { and Stomach } \\
\text { (Stage nr) }\end{array}$ & $\begin{array}{l}M=8.12 \\
\text { months } \\
\text { Range } 4 \text { to } \\
16 \text { months }\end{array}$ & $\begin{array}{l}\text { Exploratory } \\
\text { T1 = Baseline; } \\
\text { T2 = } 4 \text { weeks } \\
\text { post- } \\
\text { intervention }\end{array}$ & $\begin{array}{l}\text { Expressive } \\
\text { Writing } \\
\text { Program }\end{array}$ & $\begin{array}{l}\text { SRGS } \\
\text { T1 }(\mathrm{M}=20.68 ; \mathrm{SD}= \\
\text { 7.5613); T2 }(\mathrm{M}= \\
\text { 21.97; } \mathrm{SD}=6.6691)\end{array}$ & $\begin{array}{l}\text { Depression, } \\
\text { mood states and } \\
\text { emotions, } \\
\text { positive and } \\
\text { negative life } \\
\text { regard }\end{array}$ & $\begin{array}{l}\text { BDI } \\
\text { POMS } \\
\text { LRI }^{106}\end{array}$ & $\begin{array}{l}\text { The participants of the } \\
\text { intervention reported } \\
\text { significantly higher levels of } \\
\text { growth at } \mathrm{T} 2 \text {. }\end{array}$ \\
\hline $\begin{array}{l}\text { Labelle et } \\
\text { al. [35] }\end{array}$ & $\begin{array}{l}136 \\
\text { women } \\
\text { (75 EG; }\end{array}$ & $\begin{array}{l}\text { Breast, } \\
\text { genitourinary }\end{array}$ & $\begin{array}{l}M=23.5 \\
\text { months } \\
S D=43\end{array}$ & $\begin{array}{l}\text { Longitudinal } \\
\text { T1 = } 4 \text { weeks; }\end{array}$ & $\begin{array}{l}\text { Mindfulness } \\
\text {-Based } \\
\text { Stress } \\
\end{array}$ & $\begin{array}{l}\text { PTGI } \\
\text { MBSR group }\end{array}$ & $\begin{array}{l}\text { Spirituality, } \\
\text { facets of } \\
\text { mindfulness }\end{array}$ & $\begin{array}{l}\text { FACIT- } \\
\text { Sp } \\
\text { FFMQ }^{10}\end{array}$ & $\begin{array}{l}\text { MBSR participants } \\
\text { demonstrated increased } \\
\text { PTG, relative to CG. }\end{array}$ \\
\hline
\end{tabular}




\begin{tabular}{|c|c|c|c|c|c|c|c|c|c|}
\hline & $61 \mathrm{CG})$ & $\begin{array}{l}\text { gastrointestin } \\
\text { al, } \\
\text { lymphoma/m } \\
\text { yeloma, } \\
\text { head/neck, } \\
\text { gynaecologic } \\
\text { al, kung, } \\
\text { thyroid, } \\
\text { melanoma, } \\
\text { adrenocortica } \\
\text { l, abdominal } \\
\text { teratoma } \\
\text { adenocarcino } \\
\text { ma } \\
\text { (Stage nr) }\end{array}$ & months & $\begin{array}{l}\mathrm{T} 2=8 \text { weeks } \\
\text { after } \mathrm{T} 1\end{array}$ & $\begin{array}{l}\text { Reduction } \\
\text { (MBSR) }\end{array}$ & $\begin{array}{l}\mathrm{T} 1(M=64.87 ; S D= \\
19.25) ; \mathrm{T} 2(M=75.25 ; \\
S D=19.80) \\
\text { Control group } \\
\text { T1 }(M=57.13 ; S D= \\
24.65) ; \mathrm{T} 2(M=58.72 ; \\
S D=23.58)\end{array}$ & & 7 & $\begin{array}{l}\text { Increased in mindfulness } \\
\text { mediated the effect of } \\
\text { MBSR on PTG. }\end{array}$ \\
\hline $\begin{array}{l}\text { Pat- } \\
\text { Horenczy } \\
\text { k et al. } \\
{[50]}\end{array}$ & $\begin{array}{l}94 \\
\text { women } \\
\text { (49 EG; } \\
45 \mathrm{CG})\end{array}$ & $\begin{array}{l}\text { Breast } \\
\text { (Stage I-III) }\end{array}$ & $\mathrm{nr}$ & $\begin{array}{l}\text { Longitudinal } \\
\text { T1 = Baseline } \\
\text { (before the } \\
\text { group started); } \\
\text { T2 = } 6 \text { months } \\
\text { after T1 }\end{array}$ & $\begin{array}{l}\text { Cognitive- } \\
\text { Behaviour } \\
\text { Group } \\
\text { Intervention }\end{array}$ & $\begin{array}{l}\text { PTGI } \\
\text { EG } \\
\text { T1 }(M=3.10 ; S D= \\
1.08) ; \mathrm{T} 2(M=3.45 ; \\
S D=.79) \\
\text { CG } \\
\text { T1 }(M=3.25 ; S D= \\
1.07) ; \mathrm{T} 2(M=3.33 ; \\
S D=1.06)\end{array}$ & $\begin{array}{l}\text { PTSD } \\
\text { symptoms, } \\
\text { cognitive } \\
\text { emotion } \\
\text { regulation, }\end{array}$ & $\begin{array}{l}\text { PDS }^{108} \\
\text { CERQ }\end{array}$ & $\begin{array}{l}\text { The increase on PTG was } \\
\text { significantly greater in the } \\
\text { EG than the CG. } \\
\text { The intervention participants } \\
\text { reported more constructive } \\
\text { growth (a rise in PTG and } \\
\text { improved coping) and less } \\
\text { illusory growth (a rise in } \\
\text { PTG, but no improvement in } \\
\text { coping) than the } \\
\text { nonparticipants. }\end{array}$ \\
\hline $\begin{array}{l}\text { Stafford et } \\
\text { al. [66] }\end{array}$ & $\begin{array}{l}42 \\
\text { women }\end{array}$ & $\begin{array}{l}\text { Breast, } \\
\text { gynecologic } \\
\text { cancer } \\
\text { (cervical, } \\
\text { ovarian, } \\
\text { vaginal, } \\
\text { endometrial) } \\
\text { (Stage nr) }\end{array}$ & $\begin{array}{l}M=48.2 \\
\text { weeks } \\
\mathrm{SD}=47.0 \\
\text { weeks }\end{array}$ & $\begin{array}{l}\text { Longitudinal } \\
\text { T1 = baseline; } \\
\mathrm{T} 2=\text { post- } \\
\text { intervention; } \mathrm{T} 3 \\
=3 \text { months after } \\
\text { end of } \\
\text { intervention } \\
\text { (follow-up) }\end{array}$ & $\begin{array}{l}\text { mindfulness } \\
\text {-based } \\
\text { cognitive } \\
\text { therapy } \\
\text { (MBCT) }\end{array}$ & $\begin{array}{l}\text { PTGI } \\
\text { T1 }(M=53.50 ; S D= \\
22.31) ; \mathrm{T} 2(M=68.22 \\
S D=16.16) ; \mathrm{T} 3(M= \\
62.08 ; S D=22.75)\end{array}$ & $\begin{array}{l}\text { Distress, quality } \\
\text { of life, } \\
\text { mindfulness }\end{array}$ & $\begin{array}{l}\text { DASS - } \\
\text { SF } \\
\text { FACT- } \\
\text { G FMI - } \\
\text { SF }^{109}\end{array}$ & $\begin{array}{l}\text { PTG improved significantly } \\
\text { from } \mathrm{T} 1 \text { to } \mathrm{T} 2 \text { and from } \mathrm{T} 1 \\
\text { to } \mathrm{T} 3 \text {. }\end{array}$ \\
\hline
\end{tabular}

\section{RESULTS}

In total, 81 studies (8 entailing an intervention) were included evidencing an association of socio-demographic, health and treatment, and lifestyle characteristics with growth. Moreover, psychosocial variables (such as anxiety, depression, illness intrusiveness, positive reframing, etc.) were also found to be associated. Furthermore, studies with and without intervention will be reported separately, as well as studies that assessed PTG or BF.

\section{Empirical Studies Without Intervention}

The main characteristics and outcomes of the 73 studies are shown in table. It is important to note that the reported results focus on the main outcomes outlined by the authors and considering the more complex and comprehensive level of analysis; that is, if only univariate analysis was done, the results will mirror this information; however, if after a univariate analysis the study presents a multivariate analysis, the latter will be the only one being reported.

\section{Socio-demographic factors associated with PTG.}

The majority of studies found a significant association between age and PTG (e.g. Bellizzi et al., 2010; Brix et al., 2013; Cormio et al., 2015; Cordova et al., 2007; Danhauer et al., 2015; Danhauer et al., 2013b; Heidarzadeh et al., 2014; Kent et al., 2013; Morris et al., 2007; Mystakidou et al., 2008; Rahmani et al., 2012; Smith et al., 2014; Tanyi et al., 2015); however, other studies found an absence of correlation between both variables (Baník \& Gajdošová, 2014; Ho et al., 2011; Svetina \& Nastran, 2012). According to the findings from most studies, younger age is correlated with higher PTG (e.g. Cordova et al., 2007; Cormio et al., 2015; Danhauer et al., 2013b). Given that only few studies evaluated the relationship between PTG and gender and the results were not unanimous - some report an association with female gender (Smith et al., 2014; Tang et al., 2015) while others report an absence of association (Cormio et al., 2015; Ho et al., 2011) - this relationship remains unclear.

A higher education level is also associated with higher PTG according to most studies (e.g. Bellizzi \& Blank, 2006; Cordova et al., 2007; Danhauer et al., 2013a; Heidarzadeh et al., 2014; Rahmani et al., 2012; Smith et al., 2014; Tang et al., 2015; Wang et al., 2014); yet, a few number did not find a significant association (Cohen \& Numa, 2011; Ho et al., 2011; Mystakidou et al., 2008; Svetina \& Nastran, 2012). In what regards race or ethnicity, most studies support that this variable is associated with growth (e.g. Danhauer et al., 2015; Kent et al., 2013; Smith et al., 2008; 2014). Although there is a lack of unanimity, there is strong evidence that being married (Bellizzi \& Blank, 2006; Mystakidou et al., 2008; Ho et al., 2011); higher household income (Heidarzadeh et al., 2014; Ho et al., 2011; Wang et al., 2014); and being unemployed (Bellizzi et al., 2010) are significantly correlated with PTG.

\section{Cancer-related factors associated with PTG.}

Regarding disease-related variables, stage of cancer at diagnosis (Bellizzi et al., 2010; Ho et al., 2011; Kent et al., 2013; Smith et al., 2008; 2014), and disease/trauma severity (Morris and Shakespeare-Finch, 2011a; Posluszny et al., 2011) were positively associated with PTG.

Otherwise, a number of studies failed to find any significant association between PTG and time since diagnosis (Cohen \& Numa, 2011; Cordova et al., 2007; Cormio et al., 2015; Heidarzadeh et al., 2014; Li et al., 2015; Rahmani et al., 2012; Thombre et al., 2010); time since treatment (Cordova et al., 2007; Ho et al., 2011; Morris et al., 2007); type of treatment received (Baník \& Gajdošová, 2014; Cormio et al., 2015; Ho et al., 2011); and type of surgery (Cohen \& Numa, 2011; Silva et al., 2012; Thombre et al., 2010). Moreover, the effect of several clinical variables on PTG remains unclear, such as, type of cancer, chemotherapy, radiotherapy, hormonotherapy, comorbidities, presence/absence of metastasis and disease recurrence. 


\section{PTG IN ADULT CANCER PATIENTS: SYSTEMATIC REVIEW}

Additionally, it is important to note that very few studies with metastatic cancer patients (a sample that is difficult to invite to participate) were found, which can compromise the consistency and the generalization of the results.

\section{Physical factors associated with PTG.}

In what regards physical variables, only one study had found a negative and significant correlation between PTG and cortisol slope, indicating an association between a healthier endocrine functioning and positive psychological changes (Diaz et al., 2014).

\section{Psychosocial factors associated with PTG.}

The major part of the studies showed a significant association with growth, particularly, with a higher perception of cancer as a lifethreatening/traumatic event (Cordova et al., 2007; Smith et al., 2014) and higher perceived intensity/severity of cancer (Bellizzi \& Blank, 2006) leads to positive changes (i.e. PTG). Cancer-related intrusions or intrusive rumination were associated with higher PTG (e.g. Danhauer et al., 2013a; 2015; Soo \& Sherman, 2015; Thornton et al., 2012), which reinforces the positive association that has already been found in other studies (e.g. Cann et al., 2011).

Moreover, among mental health variables, the relationship between PTG and some factors remains unclear or inconsistent, such as depressive symptoms, distress, and PTSD symptoms, since a similar number of studies reported contrary results. Also, three studies failed to find any significant association between anxiety and PTG (Abdullah et al., 2015; Mystakidou et al., 2008; Salsman et al., 2009).

Several studies have examined the relations between growth and positive efforts or strategies to lead with a stressful traumatic event such as cancer. In fact, from the 25 studies that investigated PTG and coping, 12 of them showed that PTG is significantly associated with the following coping-related variables: positive active-adaptive coping (Bellizzi \& Blank, 2006; Danhauer et al., 2013a; Danhauer et al., 2015; Lelorain et al., 2010; Morris et al., 2007; Scrignaro et al., 2011); prognosis' acceptance-coping (Tang et al., 2015); problem-focused coping (Büyükaşik-Çolak et al., 2012; Scrignaro et al., 2011) and emotional-focused coping (Büyükaşik-Çolak et al., 2012; Thornton et al., 2012). Among all coping-related variables, many studies showed a significant positive association with positive reframing/reappraisal and growth (Bussell \& Naus, 2010; Cormio et al., 2015; Morris et al., 2007; Schmidt et al., 2011; Scrignaro et al., 2011; Thornton et al., 2012). This result is in accordance with findings from Shand and colleagues (2015). Additionally, five studies showed a positive association between religious coping and growth (Cormio et al., 2015; Lelorain et al., 2010; Rand et al., 2012; Schmidt et al., 2012). The only two studies that investigated the relationship between spirituality and growth, proved the initial hypothesis of positive correlation between both variables (Danhauer et al., 2013a; Smith et al., 2008)

PTG was significantly and positively associated with perceived social support (Bozo et al., 2009; Cohen \& Numa, 2011; Danhauer et al., 2013a; Morris and Shakespeare-Finch, 2011b; Smith et al., 2014; Tang et al., 2015; Tanriverd et al., 2012). However, two studies failed to find a significant association (Cohen \& Numa, 2011; Schmidt et al., 2012).

In recent years, and as confirmed by our results, growth has been positively associated with other positive/empowerment variables, such as happiness (Lelorain et al., 2010); satisfaction with life (Mols et al., 2009); hope (Ho et al., 2011); optimism (Bozo et al. 2009; Ho et al., 2011); openness to experience (Strack et al., 2010); and gratitude (Strack et al., 2010). Nevertheless, other two studies failed to find a relation between PTG and optimism (Bellizzi \& Blank, 2006; Smith et al., 2008) and hope (Bellizzi \& Blank, 2006).

\section{Sociodemographic factors associated with BF.}

Regarding sociodemographic features, studies failed to find significant correlations between BF and gender (Cavell et al., 2015; Harrington et al., 2008), employment status (Garland et al., 2013; Harrington et al., 2008) and marital status (Harrington et al., 2008). Alike studies with PTG, there are inconsistent findings regarding the association between $\mathrm{BF}$ and age and educational level.

\section{Clinical factors associated with BF.}

Most of the studies did not find a significant correlation between BF and time since diagnosis (Cavell et al., 2015); type of cancer (Garland et al., 2013); type of treatment (Harrington et al., 2008); and stage of cancer (Garland et al., 2013; Harrington et al., 2008; Wang et al., 2015). Though, Garland et al. (2013) and Mols et al. (2009) found significant associations between BF and time since diagnosis and stage of cancer at diagnosis, respectively.

\section{Psychosocial factors associated with BF.}

Concerning psychosocial factors the following variables were significant predictors of BF: positive active coping (Cavell et al., 2015; Kinsinger et al., 2006; Llewellyn et al., 2013); positive reappraisal (Harrington et al., 2008; Schroevers et al., 2011; Wang et al., 2015); social support (Dunn et al., 2010; Kinsinger et al., 2006; Schultz \& Mohamed, 2004); and optimism (Dunn et al., 2010; Harrington et al., 2008; Llewellyn et al., 2013). These results are in accordance with studies on PTG. Furthermore, Park et al. (2009) showed a significantly positive association between perceived benefits and religious coping, but not with religiousness. In addition, Thuné-Boyle and colleagues (2011) found that the relationship between BF and religious coping to achieve a life transformation was partially mediated by strength of faith.

Nevertheless, some studies did not find a significant correlation between BF and quality of life (Dunn et al., 2010; Kinsinger et al., 2006; Llewellyn et al., 2013), anxiety (Cavell et al., 2015; Dunn et al., 2010; Harrington et al., 2008; Llewellyn et al., 2013), and depression (Cavell et al., 2015; Harrington et al., 2008; Llewellyn et al., 2013).

\section{Comparison between groups.}

Some studies have made the comparison between patients with cancer and healthy controls regarding PTG. Most studies showed that women with breast cancer (Posluszny et al., 2011; Ruini et al., 2013; 2014; Martins da Silva et al., 2011) have higher PTG levels comparing with healthy counterparts. In contrast, a study from Brix et al. (2013) found no significant differences in PTG reported by women with breast cancer and healthy women.

Other comparisons were made between groups. As an example, in a study with women with breast cancer, Cohen and Numa (2011) found that participants who were volunteers reported similarly high levels of PTG, in comparison with non-volunteers. Also, Caucasian American women with breast cancer displayed higher PTG than African American counterparts (Bellizzi et al., 2010).

\section{PTG mediators.}

Besides the direct effects of distinct variables on PTG model, as shown by several studies, other variables have shown indirect effects on PTG, such as resilience, challenge appraisal, distress and challenge to core beliefs (e.g. Wilson et al., 2014). 
Several studies have found different PTG mediators: positive affect partially mediated the effects of general self-efficacy and expressive revealing on PTG and totally mediated the effects of emotional suppression on growth (Yu et al., 2014); cancer-related rumination partially mediated the relation between positive attentional bias and PTG (Chan et al., 2011); religiosity mediated the effect of ethnicity on PTG (Bellizzi et al., 2010); spirituality partially mediated the association between ethnicity and PTG (Smith et al., 2008); problem-focused coping fully mediated the relationship between dispositional optimism and PTG (Büyükaşik-Çolak et al., 2012); marital status moderated the relationship between the using of the combined exercise guidelines and PTG and BF (Crawford et al., 2015); trauma severity and seeking social support had a significant indirect effect on PTG (Morris \& Shakespeare-Finch, 2011b); social support given by a close person has a moderator effect in the relationship between dispositional optimism and PTG (Bozo et al., 2009); positive reframing and religious coping mediated the relationship between secure attachment and PTG (Schmidt et al., 2012).

\section{Empirical Studies with Intervention}

The support group participation has, in fact, significant effect on the increase of growth report, in accordance with some studies (e.g. Kent et al., 2013; Roepke, 2014). In this systematic review studies with interventions have been included, with the specific purpose of assessing whether the implemented programs had a significant impact on growth scores, over time.

In what regards the empirical studies with interventions, Labelle et al. (2015) (Mindfulness-Based Stress Reduction) and Pat-Horenczyk and colleagues (2015) (a cognitive-behaviour group intervention) found that the intervention group reported higher PTG than the control group. In addition, several studies found that the effect of the intervention group was significant in the post-intervention assessment (i.e. immediately after completion of the program), both on BF - Antoni et al. (2006) (Cognitive-Behavioral Stress Management) and on PTG - Hawkes et al. (2014) (Multiple Health Behaviour Change Intervention) and Stafford et al. (2013) (Mindfulness-Based Cognitive Therapy).

Moreover, the effects of the group intervention on growth were showed also longitudinally. The intervention group showed higher levels of growth at follow-up assessments, namely 4 weeks (Kállay \& Baban (2008) (Expressive Writing Program); 3 months (Stafford et al., 2013); 6 months (Cameron et al. 2007) (Psychosocial Support Program); and 12 months (Antoni et al., 2006; Cameron et al., 2007; Hawkes et al., 2014). Other study did not show a significant effect of group intervention on PTGI total score, but showed on some PTGI domains (Garlick et al., 2011) (Psycho-Spiritual Integrative Therapy).

\section{DISCUSSION}

The primary aim of this systematic review was to identify the variables associated with growth in patients with cancer diagnosis. Other systematic reviews were performed in the field of PTG and cancer; however, the objectives were different from this one. A previous systematic review entailed the psychosocial factors associated with PTG in breast cancer survivors (Kolokotroni et al., 2014) and other study reviewed PTG and PTSD among breast cancer patients (Koutroli et al., 2012). With a more comprehensive sample of participants with diverse types of cancer, Stanton and colleagues (2006) presented a systematic review about the perception of growth among cancer patients. In this study, authors selected cross-sectional and longitudinal studies and used both constructs to define growth: BF and PTG. However, the papers selected were published until 2005 and the intervention studies that have assessed PTG as an intervention outcome were not included in that review. Other systematic review and meta-analysis from Shand and colleagues (2015) analyzed, specifically, the correlations between PTG/PTSD and psychosocial and socio-demographic variables without assessing studies with intervention programs and with statistical analyses besides correlation analysis. Moreover, Roepke (2014) presented a systematic review of studies that assessed PTG as a result (primary or secondary) of a group intervention, without including other empirical studies (without intervention). Thus, the strengths of the current systematic review are: a) the inclusion of empirical studies with and without intervention and across all types of cancer; b) the inclusion of PTG, as well as similar constructs to define growth (BF, positive life changes, adversarial growth, stressrelated growth); c) the identification of both correlated and predicted variables (socio-demographic, clinical and psychosocial) of growth; d) assessment of the overall quality of the studies with and without intervention with a jury of five researchers and inter-rater agreement coefficient calculation for $80 \%$ of the studies. Therefore, the inclusion of all constructs representative of growth and all types of cancer as well as the diversity of study design will allow a wider and more informed conclusion about the correlates/predictors of growth in cancer patients.

The results of the analyzed studies indicated that PTG is associated with age, educational level, household income, stage of cancer and physical activity/exercise; is not associated with gender, number of children, type of treatment, time since treatment, time since diagnosis, and type of surgery. Despite the majority of studies confirmed the relations with these variables, some associations remained incongruent, such as the relation of PTG and marital and professional status, type of cancer as well as type, quality, and efficacy of medical treatments, as mentioned by Casellas-Grau et al. (2017).

In what regards the psychosocial variables, the majority of studies confirmed that PTG was associated with the perception of cancer as a lifethreatening event. This assumption is in accordance with the theoretical model of PTG from Tedeschi and Calhoun (1996; 2004), in which a traumatic event has to be perceived as stressfulness to trigger the challenge of core beliefs and the cognitive processing (i.e. intrusive and deliberate rumination), which in turn leads to PTG (Taku \& Oshio, 2015). Moreover, a study from Taku and Oshio (2015) supported this perspective by showing that PTG can be raised in persons that perceived low to middle levels of stress in the aftermath of the traumatic event.

In addition, PTG is associated with positive adaptive coping, problem-focused coping, emotional-focused coping, positive reframing and religious coping. In the context of cognitive processing related variables, PTG was positively associated with intrusiveness, deliberate rumination and challenge to core beliefs. Contrary to other systematic review (Kolokotroni et al., 2014), PTG seemed to be associated with both sides (intrusive vs. deliberate) of cognitive processing. However, it is noteworthy that the challenge to core beliefs, deliberate and intrusive rumination have been barely explored in the literature, since only recently these variables have been included in studies about PTG in cancer patients. Thus, we suggest the analysis of the relationship between PTG and the cognitive process in further studies. Intrusiveness (not intrusive rumination) has been evaluated in a large number of studies, proving to be positively associated with PTG (Danhauer et al., 2013a: 2015; Dunn et al., 2010; Wilson et al., 2014).

Additionally, other variables related to positive psychology were significantly associated with PTG, such as optimism, gratitude, happiness, openness to experience, hope and spirituality. However, only a reduced number of studies have explored the relations of PTG and these variables. Furthermore, findings from a systematic review of 12 studies that assessed the relationship between PTG and optimism indicated that this relationship remains unclear (Bostock et al., 2009). In this sense, more studies relating PTG and spiritual or positive outcomes are strongly suggested in order to enhance the understanding about these correlations.

Consistent to previous systematic review with a sample of breast cancer patients (Kolokotroni et al., 2014), perceived social support was positively associated with PTG, among the majority of studies (Bozo et al., 2009; Cohen \& Numa, 2011, etc). Among the types of social support, marital and family relationships have a strong influence on cancer patients' reports of growth, but only two studies (Cormio et al., 2015; Tanriverd et al., 2012) have reported significant associations between these particular type of social support and PTG. In fact, satisfactory social support provided from family members or close friends may facilitate the emotional disclosure and the cognitive processing about the traumatic experience, which in turn may potentiate higher levels of growth (Cormio et al., 2015). 


\section{PTG IN ADULT CANCER PATIENTS: SYSTEMATIC REVIEW}

Physical variables were also barely studied. Only one study reported a significant association between PTG and cortisol slope (Diaz et al., 2014). Moreover, some variables remain incongruent such as depression, distress and PTSD symptoms. The relationship between PTG and these variables was significant in some studies but not significant in others.

A minor number of studies $(n=17)$ that assessed growth as BF were found in this review. Regarding socio-demographic and clinical variables, gender, marital status, stage of cancer, type of cancer, and type of treatment were not significantly associated with BF in the most of the studies. However, it is not clear if age, educational level and time since diagnosis were significantly associated with BF, since contrary results among studies were found. In what concerns psychosocial variables, BF was associated with positive active coping, positive reappraisal, social support and optimism; and not significantly associated with quality of life, anxiety, and depression.

A comment about the differences between PTG and BF seems necessary. In this review, three studies found a positive association between BF and PTG (Baník \& Gajdošová, 2014; Li et al., 2015; Mols et al., 2009). In fact, these are two similar constructs but whose reports suggested significant content differences between them. Thus, "Reports of benefit finding might serve a more avoidant and self-protective function for individual with low personal resources (e.g. low optimism or self-efficacy) and might indicate more tangible positive change for those with more substantial resources, with distinct adaptive consequences" (Stanton et al., 2006, p.169). In addition, it is important to note that some studies have reported a specific variable (e.g. BF) but used a measurement scale that does not match the specific concept (e.g. PTGI) (e.g. Kallay \& Baban, 2008; Thornton et al., 2012). This fact confirms the difficulties encountered in the literature to define the conceptual boundaries between concepts related to growth (Stanton et al., 2006).

Regarding studies with intervention, the results suggested that the participation in group interventions may increase the report of growth. These results should be interpreted with caution, since we found a small number of studies that assessed growth as primary or secondary outcome $(n=8)$ and none of those interventions has designed an intervention to promote growth, which certainly may potentiate other conditions to facilitate the development of growth.

\section{Limitations}

This study has several limitations that need to be taken into consideration when interpreting the results. First of all, this review included only published studies, which might have affected the results obtained, since some studies that might be in course but not published may produce some interesting results that were not comprised in this review. Also, this review was limited in that only quantitative studies were included and studies that used a qualitative or mixed design were excluded. Thus, the understanding of growth in the aftermath of a trauma such as cancer may be incomplete without the reports that could be obtain with studies with qualitative methodology.

In this review, we found a small number of studies assessing BF, which may limit the comparison of predictors of PTG and BF. Also, the review of moderator analysis was based on a limited number of studies, restraining our confidence in these findings. Future research focused on mediation and moderation effects is needed.

In what regards the studies with group interventions, studies that published self-help group interventions that were moderated by a cancer patient or survivor and not by a psychologist, a nurse or a therapist, were excluded. We intended to analyze the results of interventions with a psychotherapeutic nature and objectives; however, self-help groups may also promote PTG through the modeling, "helper therapy principle" and other group processes such as self-disclosure about experiences related to cancer. Although several different constructs were included to assess growth, the amount of variables that were assessed was limited and constricted to the variables used in the studies. In this sense, psychological, cognitive and clinical variables were presented in a larger number of studies when comparing with other social or environmental variables. Further research is required to evaluate other social variables that may have impact on the level of perceived growth (e.g. health care conditions; instrumental support; number of previous traumatic events). Positive variables such as optimism, gratitude or openness to experience should also be included in further studies, since only a few studies selected in this review showed the associations of PTG and these variables. To conclude, more studies that assess growth as a result of an intervention are recommended, in order to support the evidence of the impact of group programs in the perception of positive changes after cancer.

\section{FUNDING}

This research was funded by Portuguese Foundation for Science and Technology [FCT - Fundação para a Ciência e Tecnologia], Grant/Award Number: SFRH/BD/81515/2011 and by WJCR - William James Center for Research, which is funded by FCT (grant UID/PSI/04810/2013).

\section{REFERENCES}

Abdullah, L., Iman, M. F., Nik Jaafar, N. R., Zakaria, H., Rajandram, R. K., Mahadevan, R., ... \& Shah, S. A. (2015). Posttraumatic growth, depression and anxiety in head and neck cancer patients: Examining their patterns and correlations in a prospective study. Psycho-Oncology, 24, 894-900. doi: 10.1002/pon. 3740

Antoni, M. H., Lechner, S. C., Kazi, A., Wimberly, S. R., Sifre, T., Urcuyo, K. R., ... \& Carver, C. S. (2006). How stress management improves quality of life after treatment for breast cancer. Journal of consulting and clinical psychology, 74, 1143-1152. doi: 10.1037/0022-006X.74.6.1143

APA Publications and Communications Board Working Group on Journal Article Reporting Standards (2008). Reporting standards for research in psychology: Why do we need them? What might they be? American Psychologist, 63, 848-849.

Baník, G., \& Gajdošová, B. (2014). Positive changes following cancer: posttraumatic growth in the context of other factors in patients with cancer. Supportive Care in Cancer, 22, 2023-2029. doi: /10.1007/s00520-014-2217-0

Barskova, T, \& Oesterreich, R. (2009). Post-traumatic growth in people living with a serious medical condition and its relations to physical and mental health: a systematic review. Disabil Rehabil, 31(21), 1709-1733. doi: 10.1080/09638280902738441

Bellizzi, K. M., \& Blank, T. O. (2006). Predicting posttraumatic growth in breast cancer survivors. Health Psychology, 25(1), 47-52. doi: 10.1037/0278-6133.25.1.47

Bellizzi, K. M., Smith, A. W., Reeve, B. B., Alfano, C. M., Bernstein, L., Meeske, K., ... \& Ballard-Barbash, R. R. (2010). Posttraumatic growth and health-related quality of life in a racially diverse cohort of breast cancer survivors. Journal of Health Psychology, 15(4), 615-626. doi: $10.1177 / 1359105309356364$

Bostock, L., Sheikh, A. I., \& Barton, S. (2009). Posttraumatic growth and optimism in health-related trauma: a systematic review. Journal of Clinical Psychology in Medical Settings, 16, 281-296. doi: 10.1007/s10880-009-9175-6 
Bozo, Ö., Gündoğdu, E., \& Büyükaşik-Çolak, C. (2009). The moderating role of different sources of perceived social support on the dispositional optimism - Posttraumatic growth relationship in postoperative breast cancer patients. Journal of health psychology, 14, 1009-1020. doi: $10.1177 / 1359105309342295$

Brix, S. A., Bidstrup, P. E., Christensen, J., Rottmann, N., Olsen, A., Tjønneland, A., ... \& Dalton, S. O. (2013). Post-traumatic growth among elderly women with breast cancer compared to breast cancer-free women. Acta Oncologica, 52, 345-354. doi: 10.3109/0284186X.2012.744878

Bussell, V. A., \& Naus, M. J. (2010). A longitudinal investigation of coping and posttraumatic growth in breast cancer survivors. Journal of Psychosocial Oncology, 28(1), 61-78. doi: 10.1080/07347330903438958

Büyükaşik-Çolak, C., Gündoğdu-Aktürk, E., \& Bozo, Ö. (2012). Mediating role of coping in the dispositional optimism-posttraumatic growth relation in breast cancer patients._The Journal of Psychology,_146, 471-483. doi: 10.1080/00223980.2012.654520

Cameron, L. D., Booth, R. J., Schlatter, M., Ziginskas, D., \& Harman, J. E. (2007). Changes in emotion regulation and psychological adjustment following use of a group psychosocial support program for women recently diagnosed with breast cancer. Psycho-Oncology, 16, 171-180. doi 10.1002/pon. 1050

Cann, A., Calhoun, L. G., Tedeschi, R. G., Triplett, K. N., Vishnevsky, T., \& Lindstrom, C. M. (2011). Assessing posttraumatic cognitive processes : The event related rumination inventory. Anxiety, Stress \& Coping, 24, 137-156. doi: 10.1080/10615806.2010.529901

Casellas-Grau, A., Font. A., \& Vives, J. (2014). Positive psychology interventions in breast cancer. A systematic review. Psycho-Oncology, 23, 9-19. doi: 10.1002/pon.3353

Casellas-Grau, A., Ochoa, C., \& Ruini, C. (2017). Psychological and clinical correlates of posttraumatic growth in cancer. A systematic and critical review. Psycho-oncology.1-12. doi: 10.1002/pon.4426

Cavell, S., Broadbent, E., Donkin, L., Gear, K., \& Morton, R. P. (2015). Observations of benefit finding in head and neck cancer patients. European Archives of Oto-Rhino-Laryngology, 273, 1-7. doi: 10.1007/s00405-015-3527-7

Chan, M. W., Ho, S. M., Tedeschi, R. G., \& Leung, C. W. (2011). The valence of attentional bias and cancer-related rumination in posttraumatic stress and posttraumatic growth among women with breast cancer. Psycho-Oncology, 20, 544-552. doi:10.1002/pon.1761

Cohen, M., \& Numa, M. (2011). Posttraumatic growth in breast cancer survivors: a comparison of volunteers and non-volunteers. Psycho-Oncology, 20(1), 69-76. doi: 10.1002/pon.1709

Cordova, M. J., Giese-Davis, J., Golant, M., Kronenwetter, C., Chang, V., \& Spiegel, D. (2007). Breast cancer as trauma: Posttraumatic stress and posttraumatic growth. Journal of Clinical Psychology in Medical Settings, 14, 308-319. doi: 10.1007/s10880-007-9083-6

Cormio, C., Romito, F., Giotta, F., \& Mattioli, V. (2015). Post-traumatic Growth in the Italian Experience of Long-term Disease-free Cancer Survivors. Stress and health, 31, 189-196. doi: 10.1002/smi.2545

Crawford, J. J., Vallance, J. K., Holt, N. L., \& Courneya, K. S. (2014). Associations between exercise and posttraumatic growth in gynecologic cancer survivors. Supportive Care in Cancer, 23, 705-714. doi: 10.1007/s00520-014-2410-1

Danhauer, S. C., Case, L. D., Tedeschi, R., Russell, G., Vishnevsky, T., Triplett, K., ... \& Avis, N. E. (2013). Predictors of posttraumatic growth in women with breast cancer. Psycho-Oncology, 22, 2676-2683. doi: 10.1002/pon.3298

Danhauer, S. C., Russell, G., Case, L. D., Sohl, S. J., Tedeschi, R. G., Addington, E. L., ... \& Avis, N. E. (2015). Trajectories of Posttraumatic Growth and Associated Characteristics in Women with Breast Cancer. Annals of Behavioral Medicine, 49, 650-659. doi: 10.1007/s12160-015-9696-1

Danhauer, S. C., Russell, G. B., Tedeschi, R. G., Jesse, M. T., Vishnevsky, T., Daley, K., ... \& Powell, B. L. (2013). A longitudinal investigation of posttraumatic growth in adult patients undergoing treatment for acute leukemia. Journal of clinical psychology in medical settings, 20(1),13-24. doi: 10.1007/s10880-012-9304-5

Diaz, M., Aldridge-Gerry, A., \& Spiegel, D. (2014). Posttraumatic growth and diurnal cortisol slope among women with metastatic breast cancer. Psychoneuroendocrinology, 44, 83-87. doi: 10.1016/j.psyneuen.2014.03.001

Dunn, J., Occhipinti, S., Campbell, A., Ferguson, M., \& Chambers, S. K. (2010). Benefit finding after cancer: The role of optimism, intrusive thinking and social environment. Journal of Health Psychology, 16 (1), 169-177. doi: 10.1177/1359105310371555

Garland, S. N., Valentine, D., Desai, K., Li, S., Langer, C., Evans, T., \& Mao, J. J. (2013). Complementary and alternative medicine use and benefit finding among cancer patients. The Journal of Alternative and Complementary Medicine, 19, 876-881 doi: 10.1089/acm.2012.0964

Garlick, M., Wall, K., Corwin, D., \& Koopman, C. (2011). Psycho-spiritual integrative therapy for women with primary breast cancer. Journal of Clinical Psychology in Medical Settings, 18(1), 78-90. doi: 10.1007/s10880-011-9224-9

Harding, S., Sanipour, F., \& Moss, T. (2014). Existence of benefit finding and posttraumatic growth in people treated for head and neck cancer: a systematic review. PeerJ, 2:e256. doi: 10.7717/peerj.256

Harrington, S., McGurk, M., \& Llewellyn, C. D. (2008). Positive consequences of head and neck cancer: key correlates of finding benefit. Journal of Psychosocial Oncology, 26, 43-62. doi: /10.1080/07347330802115848

Hawkes, A. L., Pakenham, K. I., Chambers, S. K., Patrao, T. A., \& Courneya, K. S. (2014). Effects of a multiple health behavior change intervention for colorectal cancer survivors on psychosocial outcomes and quality of life: A randomized controlled trial. Annals of Behavioral Medicine, 48(3), 359-370. doi: 10.1007/s12160-014-9610-2

Heidarzadeh, M., Rassouli, M., Shahbolaghi, F. M., Majd, H. A., Karam, A. M., Mirzaee, H., \& Tahmasebi, M. (2014). Posttraumatic growth and its dimensions in patients with cancer.Middle East Journal of Cancer, 5(1), 23-29. Retrived from http://mejc.sums.ac.ir/index.php/mejc/article/view/142

Ho, S., Rajandram, R. K., Chan, N., Samman, N., McGrath, C., \& Zwahlen, R. A. (2011). The roles of hope and optimism on posttraumatic growth in oral cavity cancer patients. Oral Oncology, 47, 121-124. doi: 10.1016/j.oraloncology.2010.11.015

Kállay, E., \& Baban, A. (2008). Emotional benefits of expressive writing in a sample of Romanian female cancer patients. Cognition, Brain, Behavior, 12(1), 115-129. from http://www.ascred.ro/images/attach/Emotional\%20benefits\%20of\%20expressive\%20writing\%20in\%20a\%20sample\%20of\%20romanian\%20female $\% 20$ cancer\%20patients.pdf

Kent, E. E., Alfano, C. M., Smith, A. W., Bernstein, L., McTiernan, A., Baumgartner, K. B., \& Ballard-Barbash, R. (2013). The roles of support seeking and race/ethnicity in posttraumatic growth among breast cancer survivors. Journal of Psychosocial Oncology, 31, 393-412. doi: 10.1080/07347332.2013.798759

Kinsinger, D. P., Penedo, F. J., Antoni, M. H., Dahn, J. R., Lechner, S., \& Schneiderman, N. (2006). Psychosocial and sociodemographic correlates of benefit-finding in men treated for localized prostate cancer. Psycho-Oncology,15, 954-961. doi: 10.1002/pon.1028

Kolokotroni, P., Anagnostopoulos, F., \& Tsikkinis, A. (2014). Psychosocial Factors Related to Posttraumatic Growth in Breast Cancer Survivors: A Review. Women and Health, 24, 569-592. doi: 10.1080/03630242.2014.899543

Koutroli, N., Anagnostopoulos, F., \& Potamianos, G. (2012). Posttraumatic Stress Disorder and Posttraumatic Growth in Breast Cancer Patients: A Systematic Review. Women and Health, 52, 503-516. doi: 10.1080/03630242.2012.679337 


\section{PTG IN ADULT CANCER PATIENTS: SYSTEMATIC REVIEW}

Labelle, L. E., Lawlor-Savage, L., Campbell, T. S., Faris, P., \& Carlson, L. E. (2015). Does self-report mindfulness mediate the effect of MindfulnessBased Stress Reduction (MBSR) on spirituality and posttraumatic growth in cancer patients? The Journal of Positive Psychology, 10, 153-166. doi: $10.1080 / 17439760.2014 .927902$

Lelorain, S., Bonnaud-Antignac, A., \& Florin, A. (2010). Long term posttraumatic growth after breast cancer: prevalence, predictors and relationships with psychological health. Journal of Clinical Psychology in Medical Settings, 17(1), 14-22. doi: 10.1007/s10880-009-9183-6

Li, Y. C., Yeh, P. C., Chen, H. W., Chang, Y. F., Pi, S. H., \& Fang, C. K. (2015). Posttraumatic growth and demoralization after cancer: The effects of patients' meaning-making. Palliative and Supportive Care, 13, 1449-1458. doi: 10.1017/S1478951515000048

Liberati, A., Altman, D.G., Tetzlaff, J., Mulrow, C., Gøtzsche, P.C., Ioannidis, J.P., et al. (2009). The PRISMA statement for reporting systematic reviews and meta-analyses of studies that evaluate health care interventions: Explanation and elaboration. PLoS Medicine, 6. doi: 10.1371/journal.pmed.1000100

Llewellyn, C. D., Horney, D. J., McGurk, M., Weinman, J., Herold, J., Altman, K., \& Smith, H. E. (2013). Assessing the psychological predictors of benefit finding in patients with head and neck cancer. Psycho-Oncology, 22(1), 97-105. doi: 10.1002/pon.2065

Martins da Silva, S. I., Moreira, H., \& Canavarro, M. C. (2011). Growing after breast cancer: A controlled comparison study with healthy women. Journal of Loss and Trauma, 16, 323-340. doi: 10.1080/15325024.2011.572039

Mols, F., Vingerhoets, A. J., Coebergh, J. W. W., \& van de Poll-Franse, L. V. (2009). Well-being, posttraumatic growth and benefit finding in longterm breast cancer survivors. Psychology and Health, 24(5), 583-595. doi: 10.1080/08870440701671362

Morrill, E. F., Brewer, N. T., O'Neill, S. C., Lillie, S. E., Dees, E. C., Carey, L. A., \& Rimer, B. K. (2008). The interaction of post-traumatic growth and post-traumatic stress symptoms in predicting depressive symptoms and quality of life. Psycho-Oncology, 17, 948-953. doi: 10.1002/pon.1313

Morris, B. A., \& Shakespeare-Finch, J. (2011a). Cancer diagnostic group differences in posttraumatic growth: accounting for age, gender, trauma severity, and distress. Journal of Loss and Trauma, 16, 229-242. doi: 10.1080/15325024.2010.519292

Morris, B. A., \& Shakespeare-Finch, J. (2011b). Rumination, post-traumatic growth, and distress: structural equation modelling with cancer survivors. Psycho-Oncology, 20, 1176-1183. doi: 10.1002/pon.1827

Morris, B. A., Shakespeare-Finch, J., \& Scott, J. L. (2007). Coping processes and dimensions of posttraumatic growth. The Australasian Journal of Disaster and Trauma Studies, 2007-1, 1-12. Retrieved from http://eprints.utas.edu.au/4419/

Mystakidou, K., Tsilika, E., Parpa, E., Kyriakopoulos, D., Malamos, N., \& Damigos, D. (2008). Personal growth and psychological distress in advanced breast cancer. The Breast, 17, 382-386. doi: 10.1016/j.breast.2008.01.006

Park, C. L., Edmondson, D., \& Blank, T. O. (2009). Religious and Non-Religious Pathways to Stress-Related Growth in Cancer Survivors. Applied Psychology: Health and Well-Being, 1, 321-335. doi: 10.1111/j.1758-0854.2009.01009.x

Pat-Horenczyk, R., Perry, S., Hamama-Raz, Y., et al. (2015). Posttraumatic Growth in Breast Cancer Survivors: Constructive and Illusory Aspects. Journal of Traumatic Stress, 28, 214-222. doi: 10.1002/jts.22014

Posluszny, D. M., Baum, A., Edwards, R. P., \& Dew, M. A. (2011). Posttraumatic growth in women one year after diagnosis for gynecologic cancer or benign conditions. Journal of psychosocial oncology, 29, 561-572. doi: 10.1080/07347332.2011.599360

Rahmani, A., Mohammadian, R., Ferguson, C., Golizadeh, L., Zirak, M., \& Chavoshi, H. (2012). Posttraumatic growth in Iranian cancer patients. Indian journal of cancer, 49, 287-292. doi: 10.4103/0019-509X.104489

Rand, K. L., Cripe, L. D., Monahan, P. O., Tong, Y., Schmidt, K., \& Rawl, S. M. (2012). Illness appraisal, religious coping, and psychological responses in men with advanced cancer. Supportive Care in Cancer, 20, 1719-1728. doi: 10.1007/s00520-011-1265-y

Roepke, A. M. (2014). Psychosocial interventions and posttraumatic growth: A meta-analysis. Journal of Consulting and Clinical Psychology, 83(1), 129-142. doi: $10.1037 / \mathrm{a} 0036872$

Ruini, C., Offidani, E., \& Vescovelli, F. (2014). Life Stressors, Allostatic Overload, and Their Impact on Posttraumatic Growth. Journal of Loss and Trauma, 20, 109-122. doi: 10.1080/15325024.2013.830530

Ruini, C., Vescovelli, F., \& Albieri, E. (2013). Post-traumatic growth in breast cancer survivors: new insights into its relationships with well-being and distress. Journal of clinical psychology in medical settings, 20, 383-391. doi: 10.1007/s10880-012-9340-1

Salsman, J. M., Segerstrom, S. C., Brechting, E. H., Carlson, C. R., \& Andrykowski, M. A. (2009). Posttraumatic growth and PTSD symptomatology among colorectal cancer survivors: a 3-month longitudinal examination of cognitive processing. Psycho-Oncology, 18(1), 30-41. doi: 10.1002/pon. 1367

Schmidt, S. D., Blank, T. O., Bellizzi, K. M., \& Park, C. L. (2012). The relationship of coping strategies, social support, and attachment style with posttraumatic growth in cancer survivors. Journal of Health Psychology, 17, 1033-1040. doi: 10.1177/1359105311429203

Schroevers, M. J., Kraaij, V., \& Garnefski, N. (2011). Cancer patients' experience of positive and negative changes due to the illness: relationships with psychological well-being, coping, and goal reengagement. Psycho-Oncology, 20, 165-172. doi: 10.1002/pon.1718

Schulz, U., \& Mohamed, N. E. (2004). Turning the tide: Benefit finding after cancer surgery. Social Science \& Medicine, 59, 653-662. doi: 10.1016/j.socscimed.2003.11.019

Scrignaro, M., Barni, S., \& Magrin, M. E. (2011). The combined contribution of social support and coping strategies in predicting post-traumatic growth: a longitudinal study on cancer patients. Psycho-Oncology, 20, 823-831. doi: 10.1002/pon.1782

Shand, L. K., Cowlishaw, S., Brooker, J. E., Burney, S., and Ricciardelli, L. A. (2015), Correlates of post-traumatic stress symptoms and growth in cancer patients: a systematic review and meta-analysis. Psycho-Oncology, 24, 624-634. doi: 10.1002/pon.3719

Silva, S. M., Crespo, C., \& Canavarro, M. C. (2012). Pathways for psychological adjustment in breast cancer: A longitudinal study on coping strategies and posttraumatic growth. Psychology \& Health, 27, 1323-1341. doi: 10.1080/08870446.2012.676644

Smith, B. W., Dalen, J., Bernard, J. F., \& Baumgartner, K. B. (2008). Posttraumatic growth in non-Hispanic White and Hispanic women with cervical cancer._Journal of Psychosocial Oncology,_26, 91-109. doi: 10.1080/07347330802359768

Smith, S. K., Samsa, G., Ganz, P. A., \& Zimmerman, S. (2014). Is there a relationship between posttraumatic stress and growth after a lymphoma diagnosis? Psycho-Oncology, 23, 315-321. doi: 10.1002/pon.3419

Soo, H., \& Sherman, K. A. (2015). Rumination, psychological distress and post-traumatic growth in women diagnosed with breast cancer. Psycho-Oncology, 24(1), 70-79. doi: 10.1002/pon.3596

Stafford, L., Foley, E., Judd, F., Gibson, P., Kiropoulos, L., \& Couper, J. (2013). Mindfulness-based cognitive group therapy for women with breast and gynecologic cancer: a pilot study to determine effectiveness and feasibility. Supportive Care in Cancer, 21, 3009-3019. doi: 10.1007/s00520013-1880-x

Stanton, A. L., Bower, J. E., \& Low, C. A. (2006). Posttraumatic Growth After Cancer. In L. G. Calhoun, \& R. G. Tedeschi, Handbook of Posttraumatic Growth: Research and Practice (138-175). New Jersey: Lawrence Erlbaum Associates.

Strack, J., Lopes, P., \& Gaspar, M. (2010). Reappraising cancer: life priorities and growth. Onkologie, 33, 369-374. doi: 10.1159/000315768 
Svetina, M., \& Nastran, K. (2012). Family relationships and post-traumatic growth in breast cancer patients. Psychiatria Danubina, 24, 298-306. Retrieved from traumatic+growth+in+breast+cancer+patients.+\&btnG=\&as_sdt=1\%2C5\&as_sdtp

Taku, K., \& Oshio, A. (2015). An item-level analysis of the Posttraumatic Growth Inventory: Relationships with an examination of core beliefs and deliberate rumination. Personality and Individual Differences, 86, 156-160. doi: 10.1016/j.paid.2015.06.025

Tang, S. T., Lin, K. C., Chen, J. S., Chang, W. C., Hsieh, C. H., \& Chou, W. C. (2015). Threatened with death but growing: changes in and determinants of posttraumatic growth over the dying process for Taiwanese terminally ill cancer patients. Psycho-Oncology, 24, 147-154. doi: 10.1002/pon.3616

Tanriverd, D., Savaş, E., \& Can, G. (2012). Posttraumatic growth and social support in Turkish patients with cancer. Asian Pacific Journal of Cancer Prevention, 13, 4311-4314. doi: 10.7314/APJCP.2012.13.9.4311

Tanyi, Z., Szluha, K., Nemes, L., Kovács, S., \& Bugán, A. (2015). Positive consequences of cancer: exploring relationships among posttraumatic growth, adult attachment, and quality of life. Tumori, 101, 223-231. doi: 10.5301/tj.5000244

Tedeschi, R. G., \& Calhoun, L.G. (1996). The Posttraumatic Growth Inventory: Measuring the positive legacy of trauma. Journal of Traumatic Stress, 9, 455-471. doi: 10.1002/jts.2490090305

Tedeschi, R. G., \& Calhoun, L. G. (2004). Posttraumatic growth: Conceptual foundations and empirical evidence. Psychological Inquiry, 15, 118.

Thombre, A., Sherman, A. C., \& Simonton, S. (2010). Posttraumatic growth among cancer patients in India. Journal of Behavioral Medicine, 33(1), 15-23. doi: 10.1007/s10865-009-9229-0

Thornton, A. A., Owen, J. E., Kernstine, K., Koczywas, M., Grannis, F., Cristea, M., ... \& Stanton, A. L. (2012). Predictors of finding benefit after lung cancer diagnosis. Psycho-Oncology, 21, 365-373. doi: 10.1002/pon.1904

Thuné-Boyle, C. V., Stygall, J., Keshtgar, M. R. S., Davidson, T. I., \& Newman, S. P. (2011). The influence of religious/spiritual resources on finding positive benefits from a breast cancer diagnosis. Counseling et spiritualité, 30(1), 107-134. Retrieved from http://cat.inist.fr/?aModele=afficheN\&cpsidt=24209260

Wang, M. L., Liu, J. E., Wang, H. Y., Chen, J., \& Li, Y. Y. (2014). Posttraumatic growth and associated socio-demographic and clinical factors in Chinese breast cancer survivors. European Journal of Oncology Nursing, 18, 478-483. doi: 10.1016/j.ejon.2014.04.012

Wang, Y., Zhu, X., Yang, Y., Yi, J., Tang, L., He, J., ... \& Yang, Y. (2015). What factors are predictive of benefit finding in women treated for non-metastatic breast cancer? A prospective study. Psycho-Oncology, 24, 533-539. doi: 10.1002/pon.3685

Wilson, B., Morris, B. A., \& Chambers, S. (2014). A structural equation model of posttraumatic growth after prostate cancer. Psycho-Oncology, 23, 1212-1219. doi: 10.1002/pon.3546

World Health Organization (2015). Cancer Fact sheet n²97. Available from: http://www.who.int/mediacentre/factsheets/fs297/en/. Accessed on 25 February 2016.

Yu, Y., Peng, L., Tang, T., Chen, L., Li, M., \& Wang, T. (2014). Effects of emotion regulation and general self-efficacy on posttraumatic growth in Chinese cancer survivors: assessing the mediating effect of positive affect. Psycho-Oncology, 23, 473-478. doi: 10.1002/pon.3434 